\title{
WestVirginiaUniversity
}

THE RESEARCH REPOSITORY @ WVU

Graduate Theses, Dissertations, and Problem Reports

2010

\section{Paramagnetic resonance studies of defects in titanium dioxide crystals}

\author{
Shan Yang \\ West Virginia University
}

Follow this and additional works at: https://researchrepository.wvu.edu/etd

\section{Recommended Citation}

Yang, Shan, "Paramagnetic resonance studies of defects in titanium dioxide crystals" (2010). Graduate Theses, Dissertations, and Problem Reports. 3095.

https://researchrepository.wvu.edu/etd/3095

This Dissertation is protected by copyright and/or related rights. It has been brought to you by the The Research Repository @ WVU with permission from the rights-holder(s). You are free to use this Dissertation in any way that is permitted by the copyright and related rights legislation that applies to your use. For other uses you must obtain permission from the rights-holder(s) directly, unless additional rights are indicated by a Creative Commons license in the record and/ or on the work itself. This Dissertation has been accepted for inclusion in WVU Graduate Theses, Dissertations, and Problem Reports collection by an authorized administrator of The Research Repository @ WVU.

For more information, please contact researchrepository@mail.wvu.edu. 


\title{
Paramagnetic Resonance Studies of Defects in Titanium Dioxide Crystals
}

\author{
Shan Yang \\ A Dissertation \\ Submitted to the \\ Eberly College of Arts and Sciences \\ at West Virginia University \\ in partial fulfillment of the requirements \\ for the degree of \\ Doctor of Philosophy \\ in \\ Physics
}

Larry E. Halliburton, Ph.D., Chair

Feruz Ganikhanov, Ph.D.

Nancy C. Giles, Ph.D.

James P. Lewis, Ph.D.

Nianqiang $\mathrm{Wu}, \mathrm{Ph} . \mathrm{D}$.

Department of Physics

Morgantown, West Virginia

2010

Copyright Shan Yang 2010 


\section{Abstract \\ Paramagnetic Resonance Studies of Defects in Titanium Dioxide Crystals}

\section{Shan Yang}

Electron paramagnetic resonance (EPR) and electron-nuclear double resonance (ENDOR) are used to identify and characterize point defects in $\mathrm{TiO}_{2}$ crystals having the rutile structure. Defect production occurs at low temperature during illumination with $442 \mathrm{~nm}$ laser light. Spectra with $\mathrm{S}=1 / 2$ and $\mathrm{S}=1$ are assigned to singly ionized and neutral oxygen vacancies, respectively. These oxygen vacancies have their unpaired spins localized on the two neighboring titanium ions aligned along the [001] axis. $\mathrm{A} \mathrm{Ti}^{3+}$

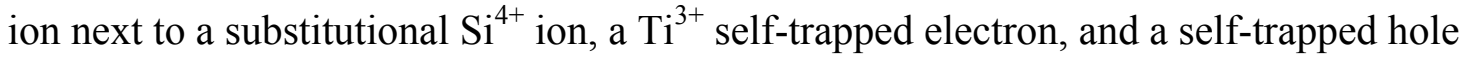
on the oxygen sublattice are also observed.

Fluorine ions substitute for oxygen and are present as unintentional impurities in $\mathrm{TiO}_{2}$ crystals. Isolated singly ionized fluorine donors in an as-grown (fully oxidized) crystal convert to their neutral charge state during exposure to $442 \mathrm{~nm}$ laser light at $6 \mathrm{~K}$. These donors return to the singly ionized charge state within a few seconds when the light is removed. In contrast, the neutral fluorine donors are observed at $6 \mathrm{~K}$ without photoexcitation after a crystal is reduced at $600{ }^{\circ} \mathrm{C}$ in flowing nitrogen gas. The angular dependences of the EPR and ENDOR spectra provide a complete set of spin-Hamiltonian parameters (principal values are $1.9746,1.9782$, and 1.9430 for the $g$ matrix and -0.23 , 0.47 , and 5.15 MHz for the ${ }^{19} \mathrm{~F}$ hyperfine matrix). These matrices suggest that the unpaired electron is localized primarily on one of the two equivalent neighboring substitutional titanium ions, i.e., the ground state of the neutral fluorine donor in rutilestructured $\mathrm{TiO}_{2}$ is a $\mathrm{Ti}^{3+}$ ion adjacent to $\mathrm{F}^{-}$ion.

Hydrogen, in the form of an $\mathrm{OH}^{-}$ion, is a shallow donor in $\mathrm{TiO}_{2}$. In the neutral charge state, the unpaired electron forms an adjacent $\mathrm{Ti}^{3+}$ ion. The hydrogen EPR signal cannot be produced in oxidized crystals containing fluorine donors, which suggest that hydrogen is a shallower donor than fluorine in $\mathrm{TiO}_{2}$ (rutile) crystals. The hydrogen EPR signal is easily observed during illumination in crystals that do not contain fluorine.

Keywords: EPR, ENDOR, $\mathrm{TiO}_{2}$, point defects, shallow donors 


\section{Acknowledgments}

I would like to acknowledge my advisor, Dr. Larry Halliburton, who has inspired me with his dedication and wisdom. He continuously conveys a spirit of passion and excitement in his research and teaching, while always remaining cautious and openminded before making any conclusions. I appreciate Dr. Halliburton offering me the opportunity to work for and with him. He guided me not only in research but offered advice in living my life in the United States. The discussions we had will never be forgotten and will be a continuing influence on both my career and life.

I would like to acknowledge the faculty for spending their valuable time to serve on my committee; Dr. Feruz Ganikhanov, Dr. Nancy Giles, Dr. James Lewis, and Dr. Nianqiang Wu. A special thanks to Dr. Giles for training me to operate optical systems, letting me use the facilities in her lab, and more importantly, for educating me in the fundamental physics behind optical properties of solid state materials.

Dr. Yongquan Jiang educated me in using experimental systems, his expertise helped me to learn effectively and efficiently. Dr. Xiaocheng Yang helped me in both academics and life, which I deeply appreciate. I thank Dr. Sean Evans, Dr. Chunchuan $\mathrm{Xu}$, and Mr. Adam Brant for very stimulating and enlightening discussions, and Dr. Pat Bunton and Dr. A. Manivannan for providing the $\mathrm{TiO}_{2}$ samples which triggered my interest in this area of study and the impetus for this dissertation. Thanks to all my friends; Ye, Yikuan, Conrad, Gaohua, Jinling, Luyi, Caiqin, Wen, Yingdong, Pengfei, Bin, Haijun, and Daolin for their support, and the many others who are not listed.

My sincerest appreciation goes to my family for their endless love and support. I especially thank my wife who is the ultimate inspiration in every aspect of my life and who is always at my side through the worst and best of times. Without the encouragement from my wife, parents, brothers, and sisters, this dissertation would not have become a reality. 
For my

Mother, Shihua

and

Father, Youfu 


\section{Table of Contents}

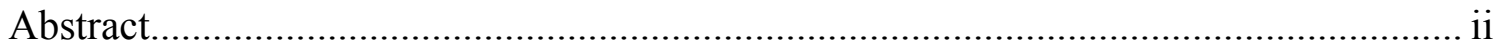

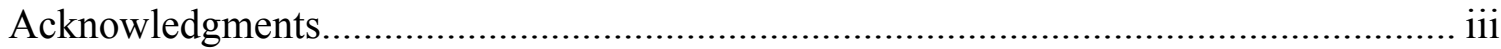

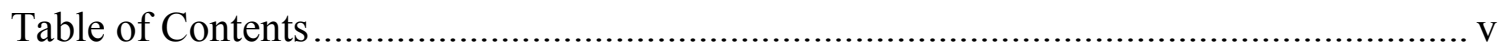

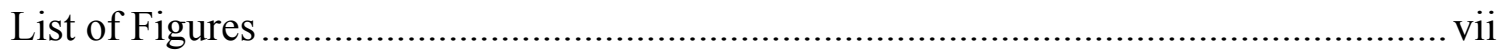

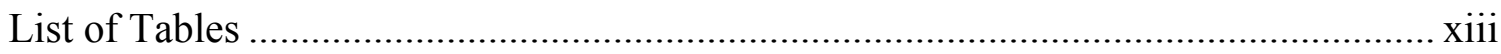

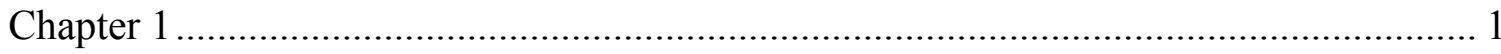

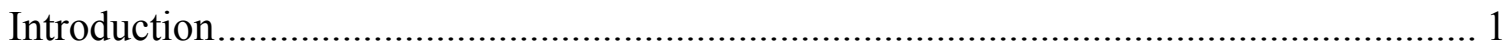

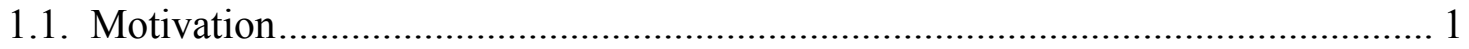

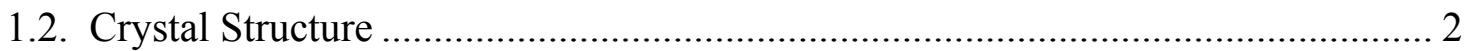

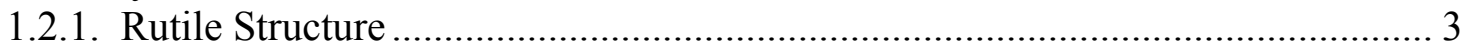

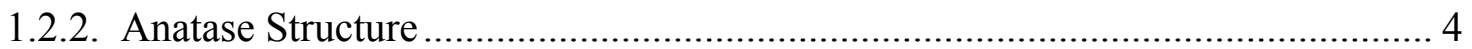

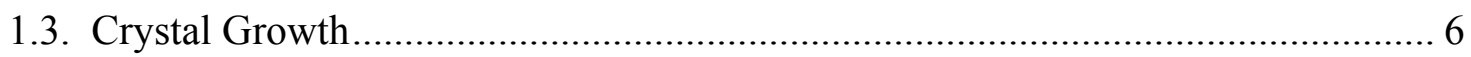

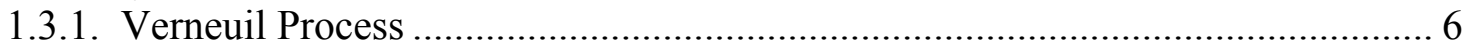

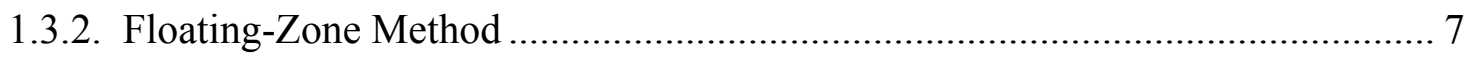

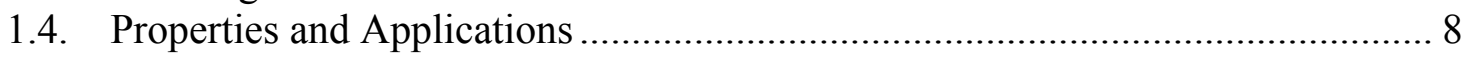

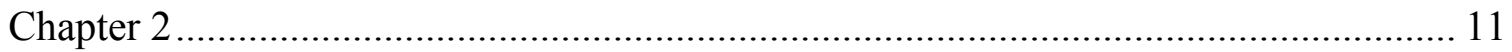

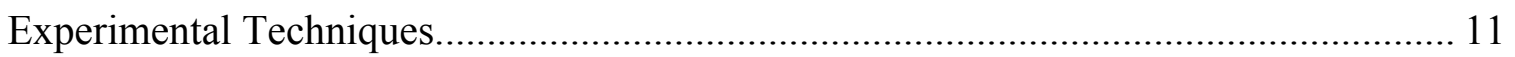

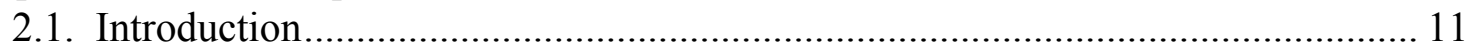

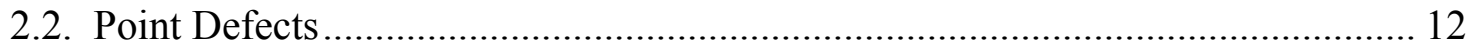

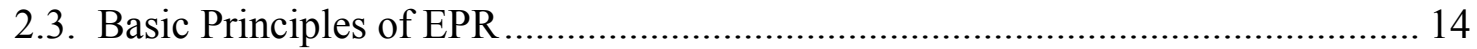

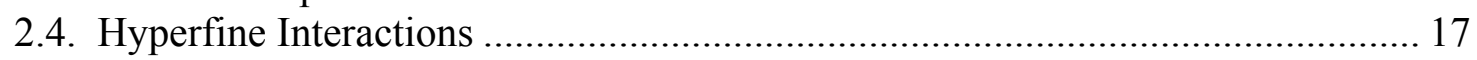

2.5. Electron-Nuclear Double Resonance ................................................................ 20

2.6. EPR and ENDOR Instruments................................................................... 22

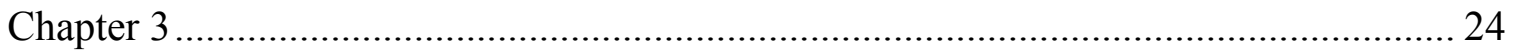

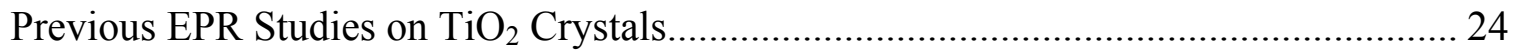

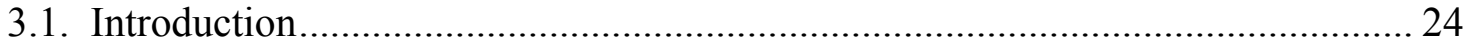

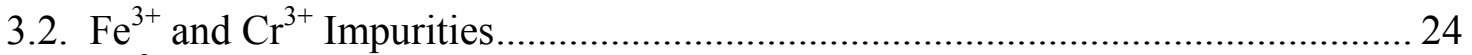

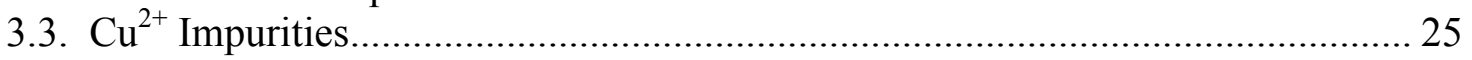

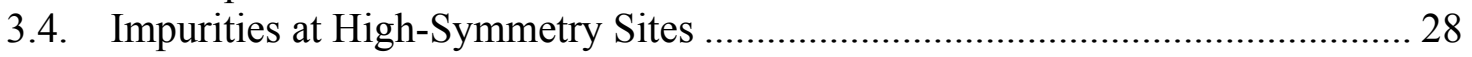

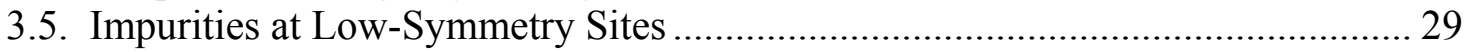

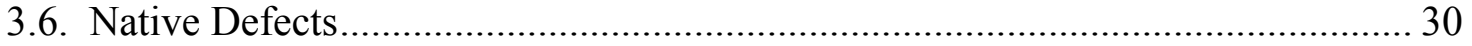

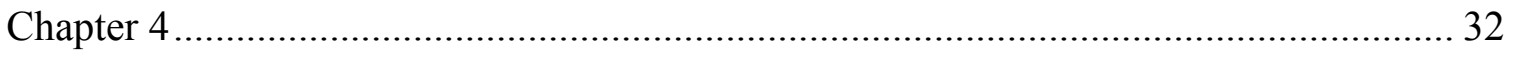




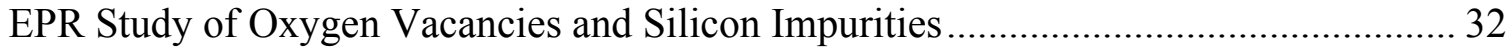

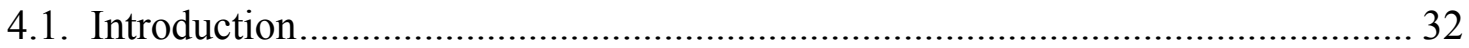

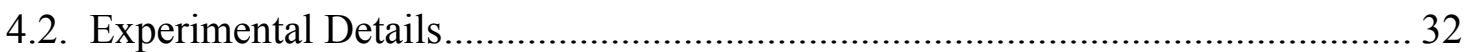

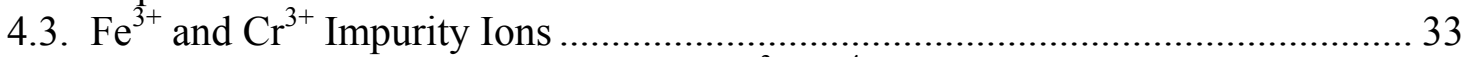

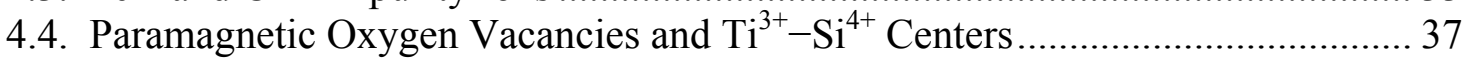

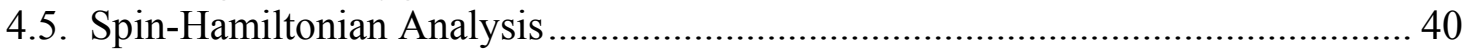

4.6. Defect Models ............................................................................................ 45

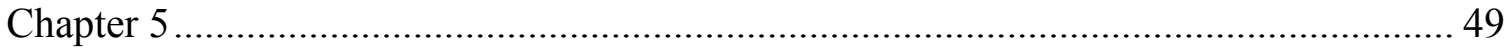

EPR Study of Self-Trapped Electron and Hole Centers ............................................. 49

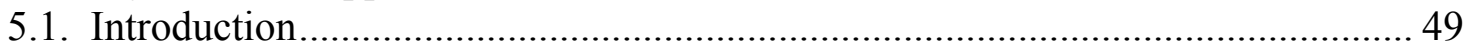

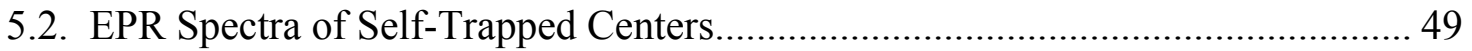

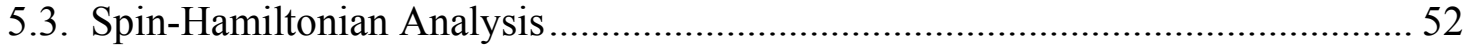

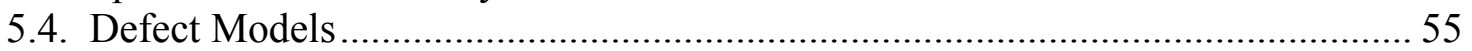

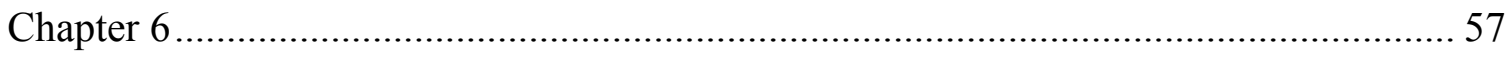

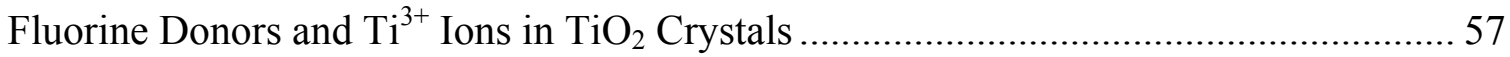

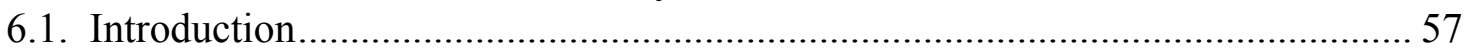

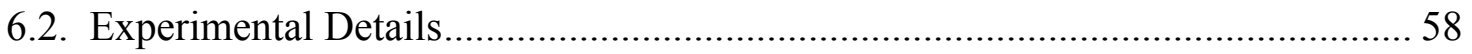

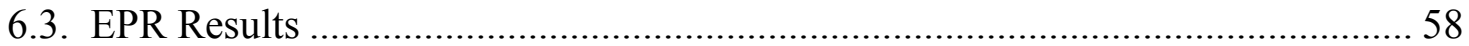

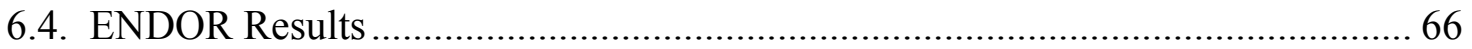

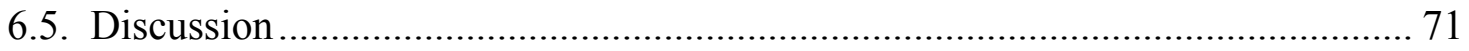

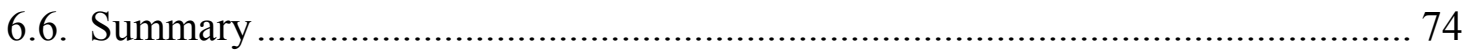

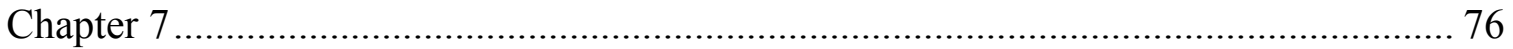

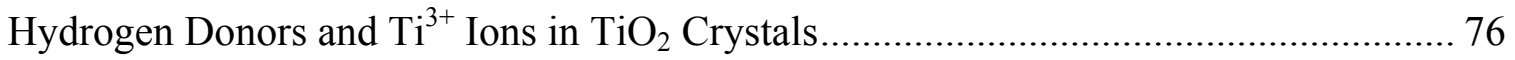

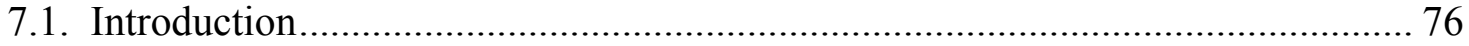

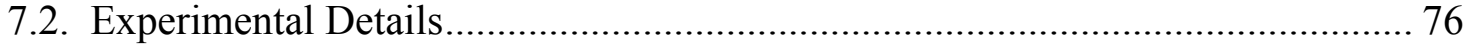

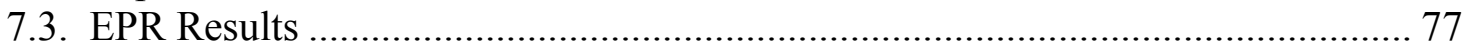

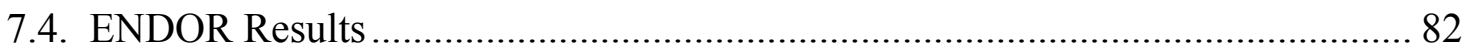

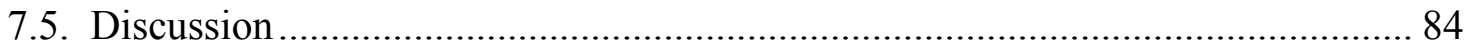

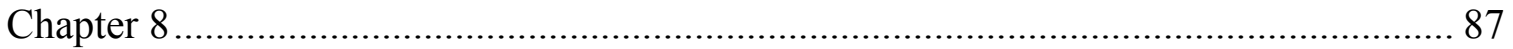

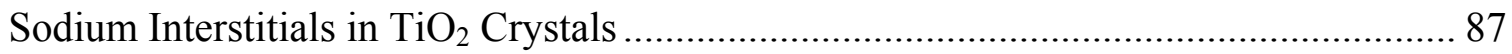

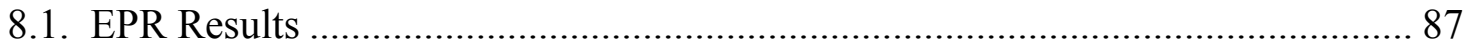

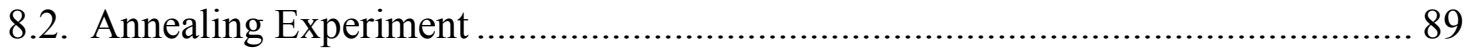

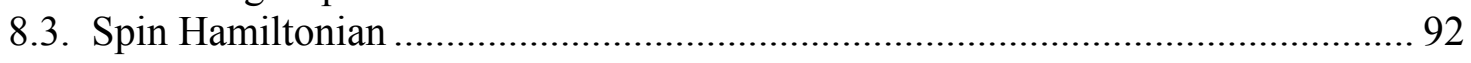

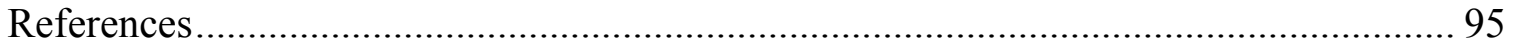

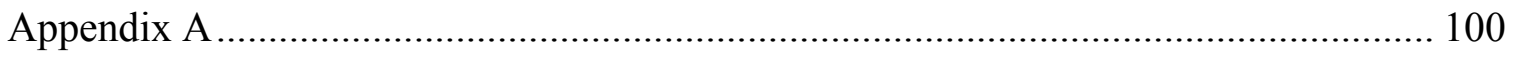

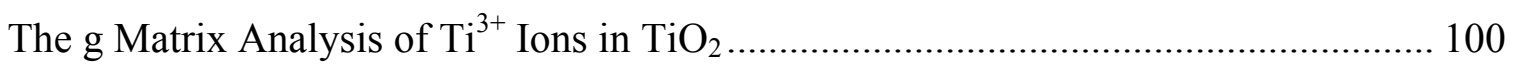

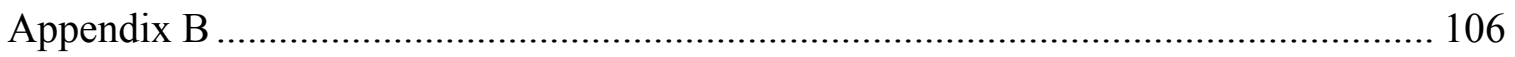




\section{List of Figures}

Figure 1.1. The unit cell of rutile-structured $\mathrm{TiO}_{2}$. Two titanium ions and four oxygen ions are included in each unit cell.

Figure 1.2. The rutile $\mathrm{TiO}_{2}$ structure in a polyhedron representation; two $\mathrm{TiO}_{6}$ octahedral units exist. Each is composed of one titanium ion at the center and six oxygen ions at the corners of the octahedron.

Figure 1.3. The unit cell of anatase-structured $\mathrm{TiO}_{2}$. There are four titanium ions and eight oxygen ions in each unit cell.

Figure 1.4. A schematic picture of the Verneuil process. After Aramgutang (taken from English Wikipedia).

Figure 1.5. A schematic representation of a four-lamp floating zone furnace. This picture was taken from the website of Dr. R. V. Jones.

Figure 1.6. A schematic illustration of the self-cleaning and self-sterilization properties

of $\mathrm{TiO}_{2}$. 10

Figure 2.1. A schematic illustration of the EPR transition for a spin $S=1 / 2$.

Figure 2.2. (a) Absorption signal representing an EPR resonance for a spin $S=1 / 2$. (b) The first derivative of the absorption signal, which represents the EPR signal.

Figure 2.3. A schematic illustration of the EPR transitions for a $S=1 / 2$ and $I=1 / 2$ spin system.

Figure 2.4. A simulated hyperfine spectrum for a $S=1 / 2$ and $I=1 / 2$ spin system.

Figure 2.5. A schematic illustration of the ENDOR transitions for a $S=1 / 2, I=1 / 2$ spin system. The red solid lines show the ENDOR transitions. The black dashed lines show the EPR transitions.

Figure 2.6. A schematic illustration of the energy splittings for a $S=1 / 2$ and $I=1 / 2$ spin system when placed in a fixed external magnetic field. 
Figure 3.1. An EPR spectrum of $\mathrm{Cr}^{3+}$ and $\mathrm{Fe}^{3+}$ ions in a $\mathrm{TiO}_{2}$ crystal. These data were taken at $30 \mathrm{~K}$ with the magnetic field along the [001] direction. The microwave frequency was near $9.59 \mathrm{GHz}$.

Figure 3.2. EPR spectra of $\mathrm{Cu}^{2+}$ ions in rutile-structured $\mathrm{TiO}_{2}$ crystals. These data were taken at $30 \mathrm{~K}$ with the magnetic field parallel to (a) the [001] direction and (b) the [110] direction. The microwave frequency was near $9.59 \mathrm{GHz}$.

Figure 4.1. EPR spectra showing hyperfine associated with $\mathrm{Cr}^{3+}$ ions in $\mathrm{TiO}_{2}$ crystals. These data were taken at $30 \mathrm{~K}$, the magnetic field was parallel to the [001] axis, and the microwave frequency was near $9.59 \mathrm{GHz}$. Trace (a) shows the hyperfine due to the ${ }^{53} \mathrm{Cr}$ nucleus $(I=3 / 2)$. Trace (b) shows the hyperfine due to the nearest neighbor ${ }^{47} \mathrm{Ti}(\mathrm{I}=5 / 2)$ and ${ }^{49} \mathrm{Ti}(\mathrm{I}=7 / 2)$ nuclei.

Figure 4.2. EPR hyperfine from $\mathrm{Fe}^{3+}$ ions in $\mathrm{TiO}_{2}$ crystals. These data were taken at 30 $\mathrm{K}$, the magnetic field was parallel to the [001] axis, and the microwave frequency was near $9.59 \mathrm{GHz}$. The two hyperfine lines separated by $12 \mathrm{G}$ are due to ${ }^{57} \mathrm{Fe}(\mathrm{I}=1 / 2)$ nuclei, and the hyperfine lines near the center line are due to ${ }^{47} \mathrm{Ti}(\mathrm{I}=5 / 2)$ and ${ }^{49} \mathrm{Ti}(\mathrm{I}=$ 7/2) nuclei.

Figure 4.3. EPR spectra taken at $26 \mathrm{~K}$ from a $\mathrm{TiO}_{2}$ crystal. The magnetic field was along the [001] direction. Trace (a) was taken with illumination of $442 \mathrm{~nm}$ laser light. Trace (b) was taken two minutes after the laser light was removed. 38

Figure 4.4. EPR spectrum of the $\mathrm{Ti}^{3+}-\mathrm{Si}^{4+}$ center taken at $26 \mathrm{~K}$ with the magnetic field parallel to the [001] direction. The upper and lower stick diagrams show the ${ }^{29} \mathrm{Si}$ and the ${ }^{47} \mathrm{Ti}$ and ${ }^{49} \mathrm{Ti}$ hyperfine lines, respectively. Additional weak lines in the spectrum are unidentified.

Figure 4.5. Angular dependence of the $\mathrm{g}$ and $\mathrm{D}$ matrices for the neutral oxygen vacancy. These EPR data were acquired in the three high-symmetry planes of the crystal. The discrete open circles are experimental data, while the solid curves are computer generated using the best fit parameters. 
Figure 4.6. Angular dependence of the g matrix for the singly ionized oxygen vacancy center. These EPR data were acquired in the three high-symmetry planes of the crystal. The discrete open circles are experimental data, while the solid curves are computer generated using the best fit parameters.

Figure 4.7. EPR angular dependence data from the $\mathrm{Ti}^{3+}-\mathrm{Si}^{4+}$ center. These EPR data were acquired in the three high-symmetry planes of the crystal. The discrete open circles are experimental data, while the solid curves are computer generated using the best fit parameters.

Figure 4.8. Structural model of an oxygen vacancy in a $\mathrm{TiO}_{2}$ crystal. (a) Upper part shows a unit cell of rutile $\mathrm{TiO}_{2}$. (b) Lower part shows the model for the neutral oxygen vacancy.

Figure 5.1. EPR spectra of the $\mathrm{STE}\left(\mathrm{Ti}^{3+}\right)$ and $\mathrm{STH}$ centers in $\mathrm{TiO}_{2}$ crystals. These data were taken at $18 \mathrm{~K}$ with the magnetic field parallel to the [001] direction. Trace (a) was taken with the $442 \mathrm{~nm}$ laser light on. Trace (b) was taken after the $442 \mathrm{~nm}$ laser light was removed.

Figure 5.2. EPR spectra of the $\mathrm{STE}\left(\mathrm{Ti}^{3+}\right)$ and $\mathrm{STH}$ centers in $\mathrm{TiO}_{2}$ crystals. These data were taken at $14 \mathrm{~K}$ with the magnetic field parallel to the [001] direction. Trace (a) was taken with the $442 \mathrm{~nm}$ laser light on. Trace (b) was taken after the $442 \mathrm{~nm}$ laser light was removed.

Figure 5.4. EPR angular dependence data of STH centers. The discrete open circles are experimental data, while the solid curves are computer generated using the best-fit $g$ matrix.

Figure 5.5. Defect model for the $\mathrm{STH}$ center in $\mathrm{TiO}_{2}$ crystals. The hole may be trapped on one oxygen ion or shared by two oxygen ions. 56

Figure 6.1. EPR spectrum of the photoinduced neutral fluorine donor in $\mathrm{TiO}_{2}$ (rutile). These data were taken at $6 \mathrm{~K}$ with the magnetic field along the [001] direction. (a) Upper spectrum was taken in the dark after cooling in the dark. (b) Lower spectrum was taken during exposure to $442 \mathrm{~nm}$ laser light. 
Figure 6.2. Production and decay of the neutral fluorine donor EPR spectrum as the 442 $\mathrm{nm}$ laser light was turned on and off. The monitoring temperature was $4.6 \mathrm{~K}$. 60

Figure 6.3. EPR spectra taken at $6 \mathrm{~K}$ after reducing a $\mathrm{TiO}_{2}$ crystal for 90 minutes at 600 ${ }^{\circ} \mathrm{C}$ in flowing nitrogen gas. The magnetic field was along the [001] direction. (a) Spectrum taken in the dark after cooling in the dark. (b) Spectrum taken during subsequent exposure to $442 \mathrm{~nm}$ laser light.

Figure 6.4. Angular dependence of the g matrix for the neutral fluorine donor in $\mathrm{TiO}_{2}$. These EPR data were acquired in the three high-symmetry planes of the crystal. Single points at the centers of the doublets are used for those angles where the fluorine hyperfine is resolved in the EPR spectra.

Figure 6.5. Angular dependence of the g matrix for the neutral fluorine donor in $\mathrm{TiO}_{2}$. These EPR data were acquired in the plane including [001] to [110] directions in the crystal. Open circles are data points and solid line are generated using best fitting $g$ matrix. Blue and black indicate two different sites.

Figure 6.6. ENDOR spectrum from the neutral fluorine donor in the $\mathrm{TiO}_{2}$ crystal, taken at $5 \mathrm{~K}$ with the magnetic field parallel to the [001] direction.

Figure 6.7. Angular dependence of the hyperfine matrix for the neutral fluorine donor in $\mathrm{TiO}_{2}$. These ENDOR data were acquired in the three high-symmetry planes of the crystal.

Figure 6.8. Model of the neutral fluorine donor illustrating the localization of the unpaired electron on the neighboring titanium ion. This is a projection on the (110) plane. The $\mathrm{g}_{1}$ and $\mathrm{A}_{1}$ principal axes are along the $[110]$ direction. 70

Figure 6.9. (a) Distorted $\mathrm{TiO}_{6}$ octahedron (the center ion is the $\mathrm{Ti}^{3+}$ ion, the six smaller dark solid circles are oxygen ions, and the eight larger shaded circles are $\mathrm{Ti}^{4+}$ ions). The $\mathrm{x}, \mathrm{y}, \mathrm{z}$ coordinate system is used in the g matrix analysis. (b) Energy ordering of the five d orbitals. 73 
Figure 7.1. The EPR spectrum of the neutral hydrogen donor in an as-received CrysTec $\mathrm{TiO}_{2}$ crystal. These data were taken at $4 \mathrm{~K}$ with the magnetic field along the [001] direction. (a) Without illumination. (b) During illumination with $442 \mathrm{~nm}$ laser light. . 78

Figure 7.2. The EPR spectrum of the neutral hydrogen donor in as-received $\mathrm{Namiki}^{\mathrm{TiO}}{ }_{2}$ crystal. These data were taken at $4 \mathrm{~K}$ with the magnetic field along the [001] direction. (a) Without illumination. (b) During illumination with $442 \mathrm{~nm}$ laser light.

Figure 7.3. The EPR spectrum of the neutral hydrogen donor in the $700{ }^{\circ} \mathrm{C}$ annealed CrysTec sample. These data were taken at $4 \mathrm{~K}$ with the magnetic field parallel to the [001] direction. The solid blue trace was taken without any illumination, while the dashed red trace was taken during illumination with $442 \mathrm{~nm}$ laser light. The two spectra are very similar.

Figure 7.4. The EPR spectrum of the neutral hydrogen donor in the $650{ }^{\circ} \mathrm{C}$ annealed MTI sample. These data were taken at $4 \mathrm{~K}$ with the magnetic field along the [001] direction. The solid blue trace was taken without any illumination, while the dashed red trace was taken during illumination with $442 \mathrm{~nm}$ laser light.

Figure 7.5. The EPR spectra of the neutral hydrogen donor in the $700{ }^{\circ} \mathrm{C}$ annealed CrysTec $\mathrm{TiO}_{2}$ crystal. These data were taken at $4 \mathrm{~K}$ with the magnetic field along (a) the [110] direction and (b) the [100] direction.

Figure 7.6. ENDOR spectrum of the neutral hydrogen donor in the $650{ }^{\circ} \mathrm{C}$ annealed MTI $\mathrm{TiO}_{2}$ crystal. These data were taken at $4 \mathrm{~K}$ with the magnetic field parallel to the [001] direction.

Figure 7.7. ENDOR spectrum of the neutral hydrogen donor in the $650{ }^{\circ} \mathrm{C}$ annealed MTI $\mathrm{TiO}_{2}$ crystal. These data were taken at $4 \mathrm{~K}$ with the magnetic field parallel to the [110] direction.

Figure 7.8. ENDOR spectrum of the neutral hydrogen donor in the $650{ }^{\circ} \mathrm{C}$ annealed MTI $\mathrm{TiO}_{2}$ crystal. These data were taken at $4 \mathrm{~K}$ with the magnetic field parallel to the [100] direction. 
Figure 8.1. The four-line EPR spectrum taken from an as-received $\mathrm{MTI} \mathrm{TiO}_{2}$ crystals These data were taken at $30 \mathrm{~K}$ with the magnetic field along the [001] direction in the crystal.

Figure 8.2. The four-line EPR spectrum taken from an as-received $\mathrm{MTI} \mathrm{TiO}_{2}$ crystals. These data were taken at $30 \mathrm{~K}$ with the magnetic field along (a) the [110] direction and (b) the [100] direction in the crystal.

Figure 8.3. The four-line pattern EPR spectra taken from (a) the as-received MTI sample and (b) the $\mathrm{NaOH}$ annealed MTI sample. These data were taken at $30 \mathrm{~K}$ with the magnetic field along the [001] direction. 90

Figure 8.4. The four-line pattern EPR spectra taken from (a) the as-received MTI sample and (b) the $\mathrm{LiOH}$ annealed MTI sample. These data were taken at $30 \mathrm{~K}$ with the magnetic field along the [001] direction.

Figure 8.5. Angular dependence of the $g$ matrix and $\mathrm{D}$ matrix for the $\mathrm{Na}^{+}-\mathrm{V}_{\mathrm{O}}^{0}$ center in $\mathrm{TiO}_{2}$. These EPR data were acquired in the two high-symmetry planes of the crystal. The open circles are experimental data, and the solid curves are generated using the bestfit $g$ and $\mathrm{D}$ matrices. 


\section{List of Tables}

Table 3.1. Spin-Hamiltonian parameters of $\mathrm{Cu}^{2+}$ in $\mathrm{TiO}_{2}$ (rutile) single crystals. These values are taken from References 3.3 and 3.4.

Table 3.2. Spin-Hamiltonian parameters of impurities in $\mathrm{TiO}_{2}$ crystals. 28

Table 3.3. Spin-Hamiltonian parameters of defects associated with trivalent impurities in $\mathrm{TiO}_{2}$ crystals.

Table 4.1. Spin-Hamiltonian parameters of the $\mathrm{V}_{\mathrm{O}}^{0}$ center and the $\mathrm{V}_{\mathrm{O}}^{+}$center. These parameters are generated by fitting the EPR angular dependence data using a leastsquares MATLAB program). The angle $\phi$ is relative to the [100] direction.

Table 5.1. Spin-Hamiltonian parameters for the self-trapped electron center and the selftrapped hole center.

Table 6.1. Spin-Hamiltonian parameters for the neutral fluorine donor in a $\mathrm{TiO}_{2}$ (rutile) crystal. The [110] principal axes of $\mathrm{g}_{1}$ and $\mathrm{A}_{1}$ are arbitrarily defined to be parallel to the elongation direction of a distorted $\mathrm{TiO}_{6}$ octahedron (see Section 6.3). Directions of the principal axes for $A_{2}$ and $A_{3}$ are in the (110) plane.

Table 8.1. Spin-Hamiltonian parameters of the $\mathrm{Na}^{+}-\mathrm{V}_{\mathrm{O}}^{0}$ center. These parameters are generated by analyzing the EPR angular dependence data using a least-squares fitting MATLAB program. 


\section{Chapter 1}

\section{Introduction}

\subsection{Motivation}

Titanium dioxide, with the chemical formula $\mathrm{TiO}_{2}$, is a versatile transition-metal oxide. This compound is found in nature in three different crystalline forms (rutile, anatase, and brookite). Rutile and anatase are common and used widely, while brookite is rare and so far is less used. Titanium dioxide $\left(\mathrm{TiO}_{2}\right)$ is a wide-band-gap semiconductor with many applications ranging from solar energy conversion to self-cleaning hygienic surfaces $[1.1-1.4]$.

Although $\mathrm{TiO}_{2}$ has been widely studied for more than five decades, a detailed understanding of the role of point defects in controlling its optical and electrical properties is still emerging [1.5-1.7]. In 1972, Fujishima and Honda [1.8] found that $\mathrm{TiO}_{2}$ can decompose water without external electric power. This discovery brought increased attention to $\mathrm{TiO}_{2}$ and greatly expanded the research activity on this material. In the ensuing years, researchers realized that $\mathrm{TiO}_{2}$ had the potential to serve as a photocatalyst, a dyesensitized solar cell, and a gas sensor [1.9]. Among these applications, photocatalysis is very important and has been intensively investigated. As a versatile photcatalyst, $\mathrm{TiO}_{2}$ can be used for deodorizing, self-cleaning, self-sterilizing, and electrolytic splitting of water into hydrogen and oxygen. Asahi [1.10] discovered in 2001 that doping $\mathrm{TiO}_{2}$ with nitrogen increased its visible-light activity. These observations renewed the interest in improving the performance of devices containing $\mathrm{TiO}_{2}$. In the last few years, more than two thousand papers have been published annually on this material.

An important strategy presently being used by many investigators to optimize $\mathrm{TiO}_{2}$ for specific applications involves modification of the optical and electrical properties of this material by adding or removing selected donors and acceptors (impurities as well as native defects). Success in these efforts, however, requires a complete under- 
standing of the fundamental characteristics of the donors and acceptors $\mathrm{in}^{\mathrm{TiO}_{2}}$, including their electronic structure and optical responses. Although much of the recent experimental work in $\mathrm{TiO}_{2}$ has focused on powders, thin films, nanoparticles, nanotubes, nanowires, etc., it is more informative to use bulk crystals to initially and fully investigate the point defects. Electron paramagnetic resonance (EPR) and electron-nuclear double resonance (ENDOR) techniques are well suited to study paramagnetic point defects in bulk crystals [1.4,1.11]. Information obtained from a bulk crystal can then be used to interpret the EPR spectra observed in various $\mathrm{TiO}_{2}$ powders and nanostructures.

Nearly all of the early fundamental studies of $\mathrm{TiO}_{2}$ were carried out on rutilestructured crystals. This is the structure of all currently available commercial bulk single crystals of $\mathrm{TiO}_{2}$. Rutile is also the most stable form among three $\mathrm{TiO}_{2}$ structures. Studies of the physical properties of $\mathrm{TiO}_{2}$ anatase emerged in 1990s, when high purity and large single crystals of anatase were successfully grown.

The present dissertation focuses on paramagnetic point defects in $\mathrm{TiO}_{2}$ (rutile) single crystals. Detailed EPR and ENDOR studies are described for a series of defects.

\subsection{Crystal Structure}

The three different structures of titanium dioxide $\left(\mathrm{TiO}_{2}\right)$ crystals (i.e., rutile, anatase, and brookite) are referred to as the high-temperature-stable form, the lowtemperature-stable form, and the metastable form, respectively. Rutile is the most stable crystal form and is therefore the most common. Single crystals of rutile are readily available from commercial crystal growers. Single crystals of anatase are rare due to their extremely difficult growth conditions, but anatase powders or nanoparticles are easy to synthesize and anatase thin films are easy to grow. The anatase structure transforms into rutile during heating. The temperature to change anatase to rutile is $915^{\circ} \mathrm{C}$, and the change is nonreversible. 


\subsubsection{Rutile Structure}

Figure 1.1 shows a unit cell of $\mathrm{TiO}_{2}$ (rutile). This material exhibits tetragonal symmetry and belongs to space group $P 4_{2} / \mathrm{mnm}$. Its lattice constants are $a=4.5937 \AA$ and $c=2.9587 \AA$. The ionic radius of the $\mathrm{Ti}^{4+}$ ion is $0.60 \AA$, while the ionic radius of the $\mathrm{O}^{2-}$ ion is $1.40 \AA$. The density of $\mathrm{TiO}_{2}$ (rutile) is $4.27 \mathrm{~g} \mathrm{~cm}^{-3}$, which is the largest value of density among the three $\mathrm{TiO}_{2}$ crystal structures. In each primitive unit cell of $\mathrm{TiO}_{2}$ (rutile), there are two $\mathrm{Ti}^{4+}$ ions. One $\mathrm{Ti}^{4+}$ ion lies at the center of the unit cell and one $\mathrm{Ti}^{4+}$ ion occupies a corner of the unit cell. $\mathrm{A} \mathrm{Ti}^{4+}$ ion at the corner of the unit cell is shared by eight unit cells. Similarly, there are four $\mathrm{O}^{2-}$ ions in each primitive unit cell. In $\mathrm{TiO}_{2}$ (rutile), each $\mathrm{Ti}^{4+}$ is surrounded by six $\mathrm{O}^{2-}$ ions. These six oxygen ions form a distorted octahedron with a titanium ion at its center. The $\mathrm{Ti}^{4+}$ ion at the center of a unit cell is crystallographically different from the $\mathrm{Ti}^{4+}$ ions at the corners of the unit cell

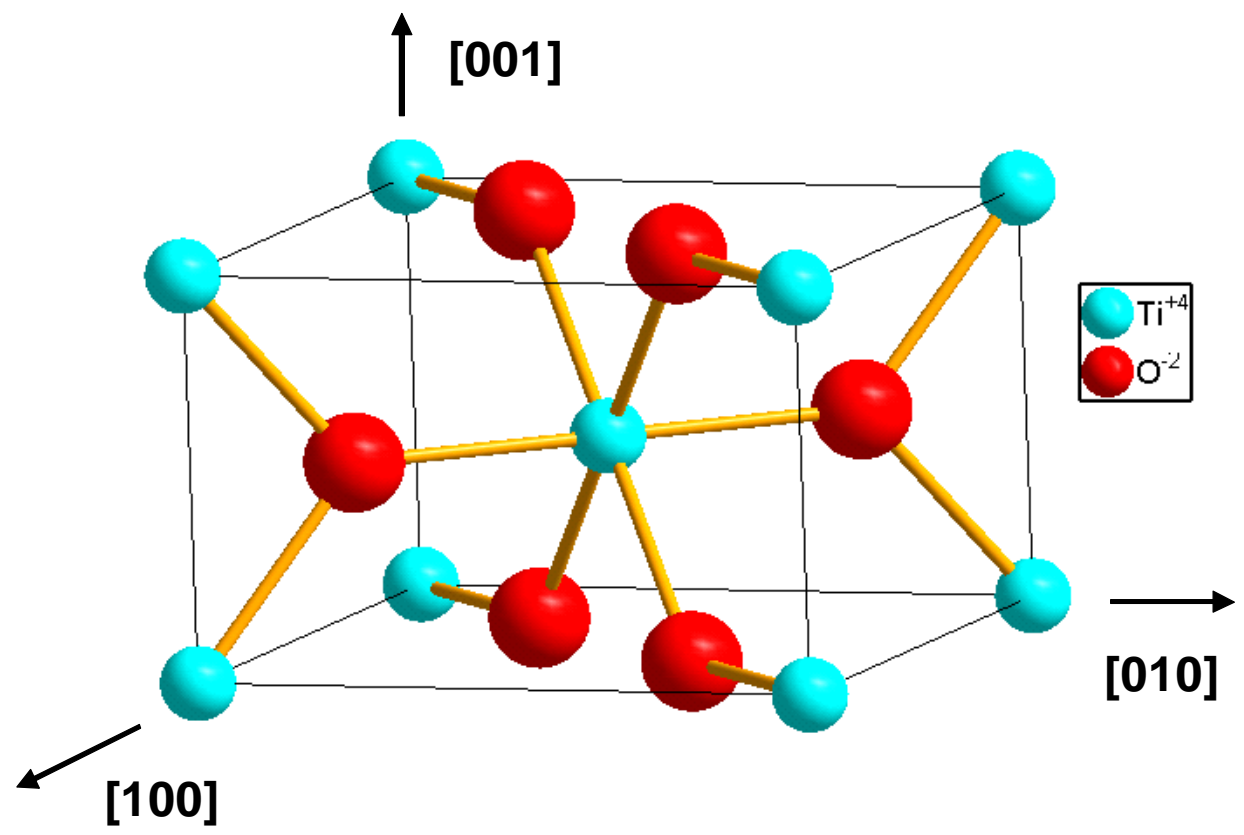

Figure 1.1. The unit cell of rutile-structured $\mathrm{TiO}_{2}$. Two titanium ions and four oxygen ions are included in each unit cell. 


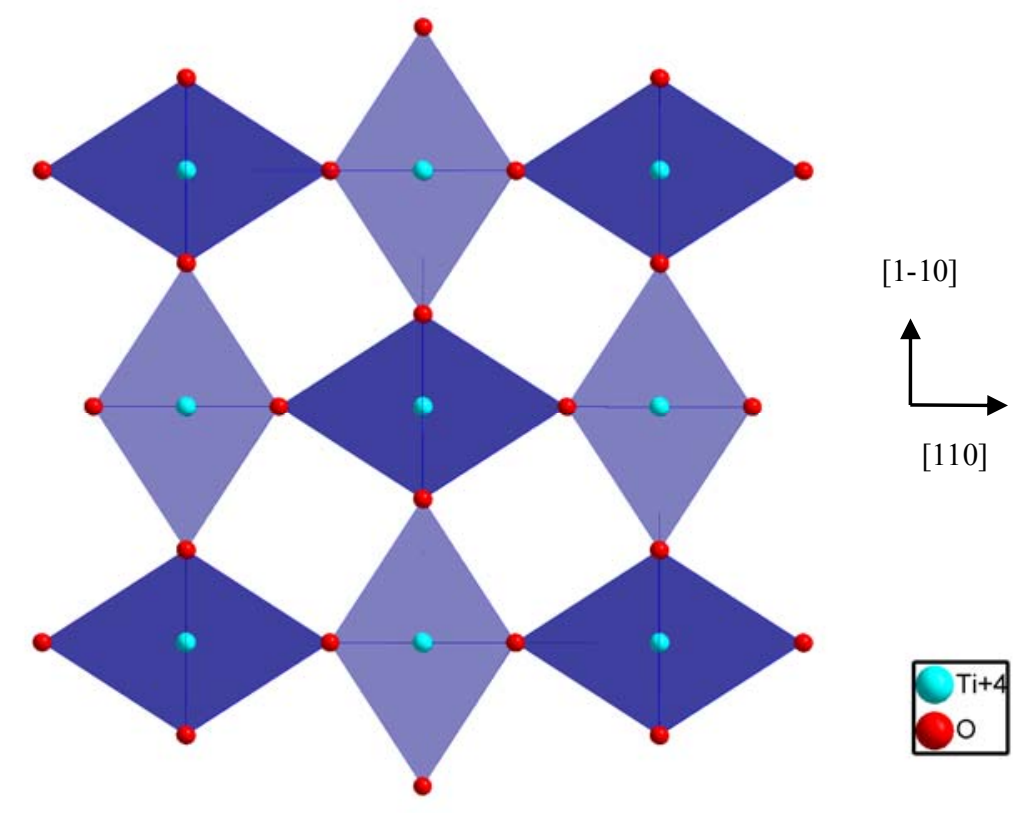

Figure 1.2. The rutile $\mathrm{TiO}_{2}$ structure in a polyhedron representation; two $\mathrm{TiO}_{6}$ octahedral units exist. Each is composed of one titanium ion at the center and six oxygen ions at the corners of the octahedron.

(as shown in Fig. 1.1). These two $\mathrm{Ti}^{4+}$ ions represent different sites, and thus paramagnetic defects occupying these sites become magnetically inequivalent for an arbitrary direction of magnetic field. These two sites for $\mathrm{Ti}^{4+}$ ions result in two geometrically different octahedra, which transform into each other by rotating $90^{\circ}$ around the $c$ axis followed by a shift of $\mathrm{c} / 2$ along the $c$ axis. Figure 1.2 shows the polyhedron representation of the $\mathrm{TiO}_{2}$ (rutile) structure. Two spatially different octahedrons are easily recognized. Each octahedron is composed of one titanium ion at the center and six oxygen ions at the six corners.

\subsubsection{Anatase Structure}

Figure 1.3 shows a unit cell of the $\mathrm{TiO}_{2}$ (anatase) crystal structure. The anatase structure is similar to the rutile structure; they both have tetragonal symmetry, but the 
space group of anatase is $I 4_{1} /$ amd. Similar structures for anatase and rutile result in nearly the same properties of $\mathrm{TiO}_{2}$ (anatase) and $\mathrm{TiO}_{2}$ (rutile), such as density, hardness, and luster. The lattice constants for anatase are $a=3.7845 \AA$ and $c=9.5143 \AA$. The density of anatase is $3.90 \mathrm{~g} \mathrm{~cm}^{-3}$, which is close to but slightly smaller than the density of rutile. The distortion of a $\mathrm{TiO}_{6}$ octahedron in anatase is slightly larger than that found in rutile, and this results in longer bond lengths along the [001] direction than those near the basal plane (perpendicular to [001]).

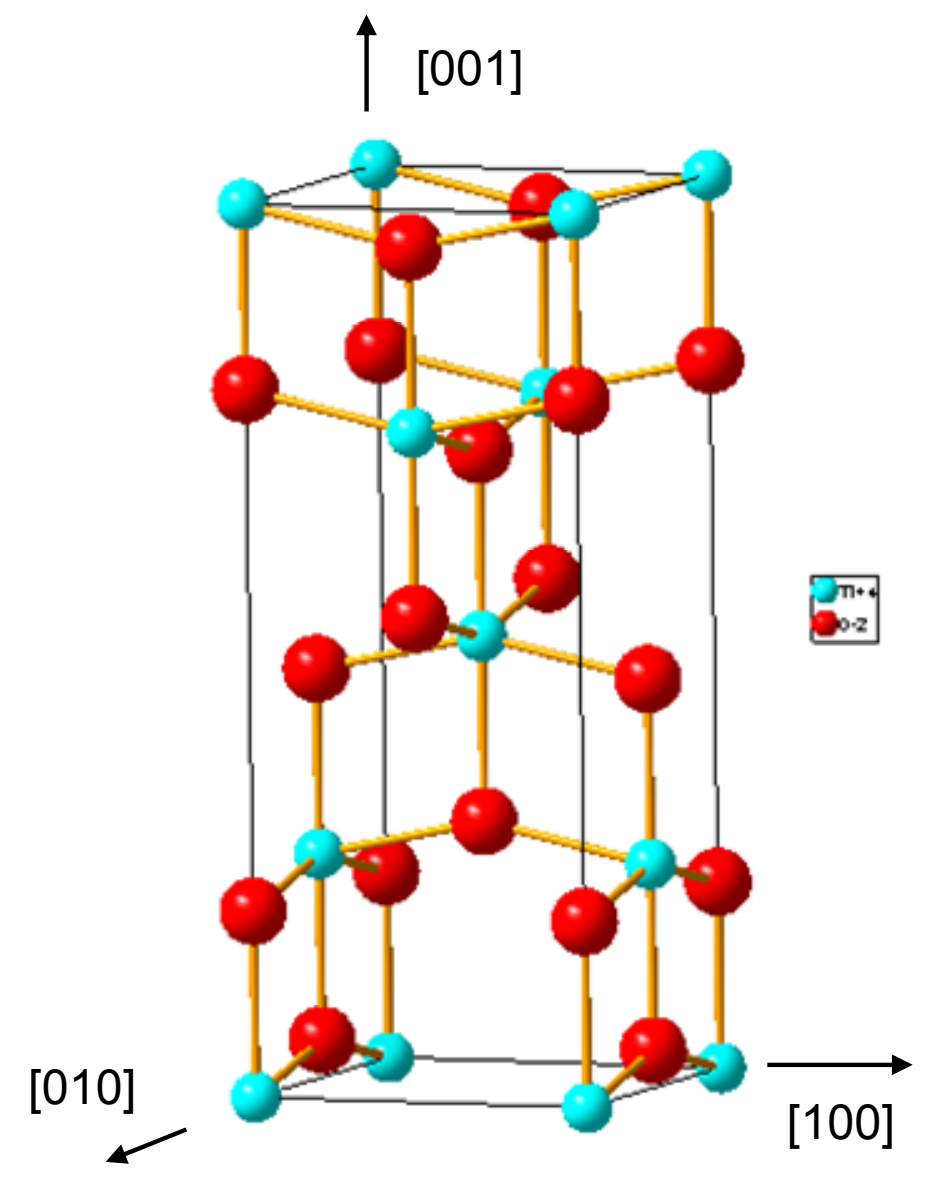

Figure 1.3. The unit cell of anatase-structured $\mathrm{TiO}_{2}$. There are four titanium ions and eight oxygen ions in each unit cell. 


\subsection{Crystal Growth}

The starting materials used to grow single crystals of $\mathrm{TiO}_{2}$ are often derived from ilmenite ore (less than $60 \% \mathrm{TiO}_{2}$ ) or rutile ore (about $95 \% \mathrm{TiO}_{2}$ ) A common method to obtain high-purity starting materials in preparation for growth is to use $\mathrm{HCl}$ or $\mathrm{HF}$ acid to extract $\mathrm{TiO}_{2}$ from ore. In this process, $\mathrm{TiCl}_{4}$ and $\mathrm{TiF}_{4}$ are the intermediate products, and they sublime at temperature lower than $300{ }^{\circ} \mathrm{C}$. High-purity $\mathrm{TiO}_{2}$ powders may be made by this method with a few additional steps (washing, drying, heating, etc.) $[1.12,1.13]$. The commercially available $\mathrm{TiO}_{2}$ (rutile) single crystals are mainly grown in two ways: the Verneuil process and the floating-zone method.

\subsubsection{Verneuil Process}

The Verneuil process, also called the flame fusion process, was developed by a French chemist, Auguste Verneuil. The originally purpose of this method was to produce rubies and sapphires. It was later introduced to grow single crystals of rutile, strontium titanate, YAG, cubic zirconia, etc. [1.14]. Figure 1.4 illustrates schematically the basic features of this method.

A Verneuil furnace has two major parts, an upper feedstock container and a lower combustion chamber containing a candle-shaped ceramic support for the seed crystal. The feedstock container at the top of the furnace holds highly purified $\mathrm{TiO}_{2}$ powder and has two small tubes connected to it. One tube located on the side near the top allows oxygen gas to be fed into the container. Another tube located at the bottom allows the $\mathrm{TiO}_{2}$ powders and the oxygen gas to leave the upper container when the container vibrates. The $\mathrm{TiO}_{2}$ powders and the oxygen gas enter the combustion chamber which is immediately below the upper container. Hydrogen gas is supplied to the combustion chamber and ignited when meeting the oxygen gas. The temperature at the flame point above the seed crystal is near the melting point of $1840{ }^{\circ} \mathrm{C}$ when growing $\mathrm{TiO}_{2}$ crystals. $\mathrm{TiO}_{2}$ powders melt at this flame point and form a droplet which falls onto the top of the 


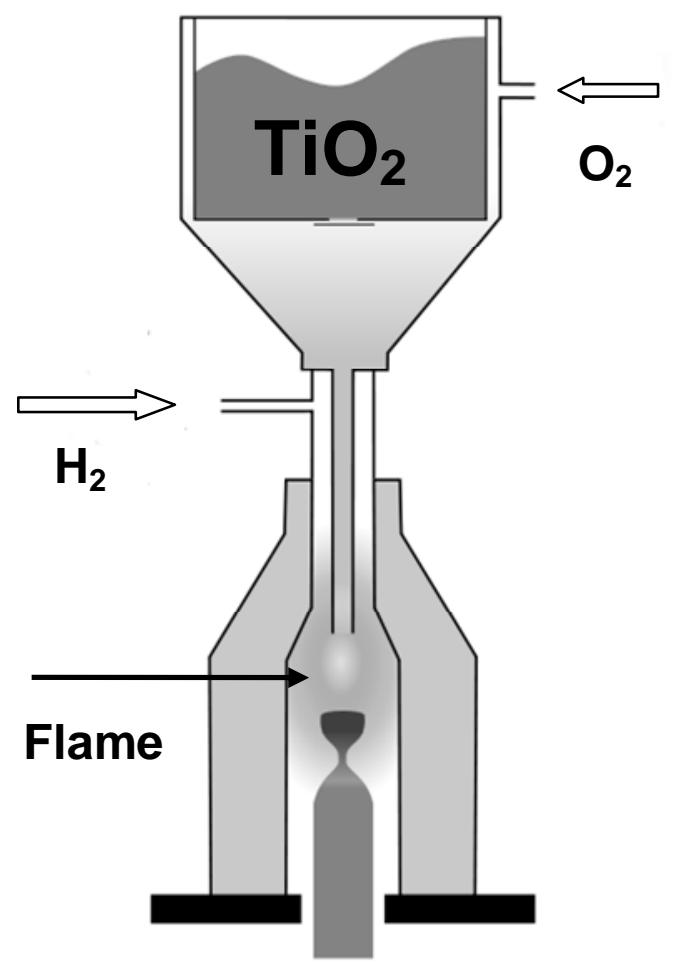

Figure 1.4. A schematic picture of the Verneuil process. After Aramgutang, (taken from English Wikipedia).

$\mathrm{TiO}_{2}$ seed crystal (pre-placed on top of the candle-shaped ceramic support). As more droplets fall, the crystal starts growing. Moving the support for the seed crystal down slowly allows the crystal to grow to a large size.

\subsubsection{Floating-Zone Method}

Several groups, starting in the 1960s, have used the floating-zone method to grow $\mathrm{TiO}_{2}$ crystals $[1.15,1.16]$. This method is very similar to the Verneuil process. In both cases, the starting material is $\mathrm{TiO}_{2}$ powder. In the floating zone method, instead of a flame, thermal radiation from lamps (such as halogen arc lamps or infrared lamps) is reflected by ellipsoidal mirrors and focused in the center zone. A floating zone furnace may have two lamps (with two mirrors) or four lamps (with four mirrors). Figure 1.5 


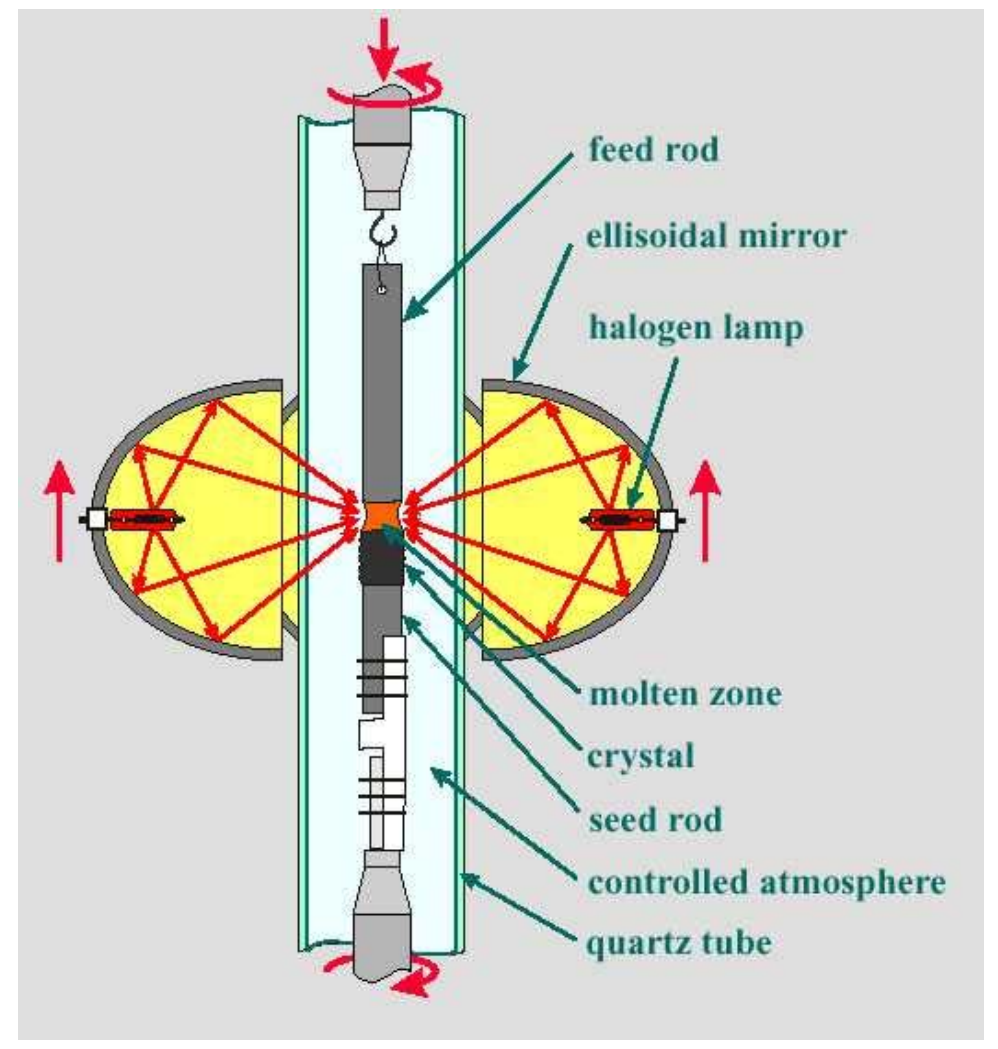

Figure 1.5. A schematic representation of a four-lamp floating zone furnace. This picture was taken from the website of Dr. R. V. Jones.

shows a schematic illustration of a floating zone furnace.

\subsection{Properties and Applications}

$\mathrm{TiO}_{2}$ has unique and versatile electric, catalytic, and electrochemical properties, and its three different crystalline modifications further expand these properties. One widespread, but low-tech, application of $\mathrm{TiO}_{2}$ is as a paint pigment because of its high refractive index [1.17]. $\mathrm{TiO}_{2}$ has an unusually high dielectric constant compared to many other semiconductors [1.18]. At room temperature, the static dielectric constant for the [001] direction in $\mathrm{TiO}_{2}$ (rutile) is 170. Advanced CMOS technology requires the gate dielectric $\mathrm{SiO}_{2}$ to have a thickness of a few monolayers, which result in large current leakage. This leakage may be minimized if a large dielectric constant material such as 
$\mathrm{TiO}_{2}$ is used to replace $\mathrm{SiO}_{2}$. In an oxygen-deficient environment, $\mathrm{TiO}_{2}$ tends to loose oxygen and its electrical resistance changes. This effect makes $\mathrm{TiO}_{2}$ a good candidate for oxygen sensor devices.

At room temperature, the band gap is $3.0 \mathrm{eV}$ for $\mathrm{TiO}_{2}$ (rutile) and $3.2 \mathrm{eV}$ for $\mathrm{TiO}_{2}$ (anatase). Illuminating $\mathrm{TiO}_{2}$ with above-band-gap ultraviolet (uv) light generates many electron-hole pairs by moving electrons from the valence band to the conduction band.

$$
\mathrm{uv} \rightarrow \mathrm{e}^{-}+\mathrm{h}^{+}
$$

In the presence of water, a series of reactions may occur that leads to the most important application of $\mathrm{TiO}_{2}$, i.e., photocatalysis. The following are possible reactions:

$$
\begin{aligned}
& \mathrm{O}_{2}+\mathrm{e}^{-} \rightarrow \mathrm{O}^{2-} \\
& \mathrm{H}_{2} \mathrm{O}+\mathrm{h}^{+} \rightarrow \cdot \mathrm{OH}+\mathrm{H}^{+} \text {(where } \cdot \mathrm{OH} \text { indicates an } \mathrm{OH} \text { radical) } \\
& \mathrm{O}_{2}+2 \mathrm{H}^{+}+2 \mathrm{e}^{-} \rightarrow \mathrm{H}_{2} \mathrm{O}_{2}
\end{aligned}
$$

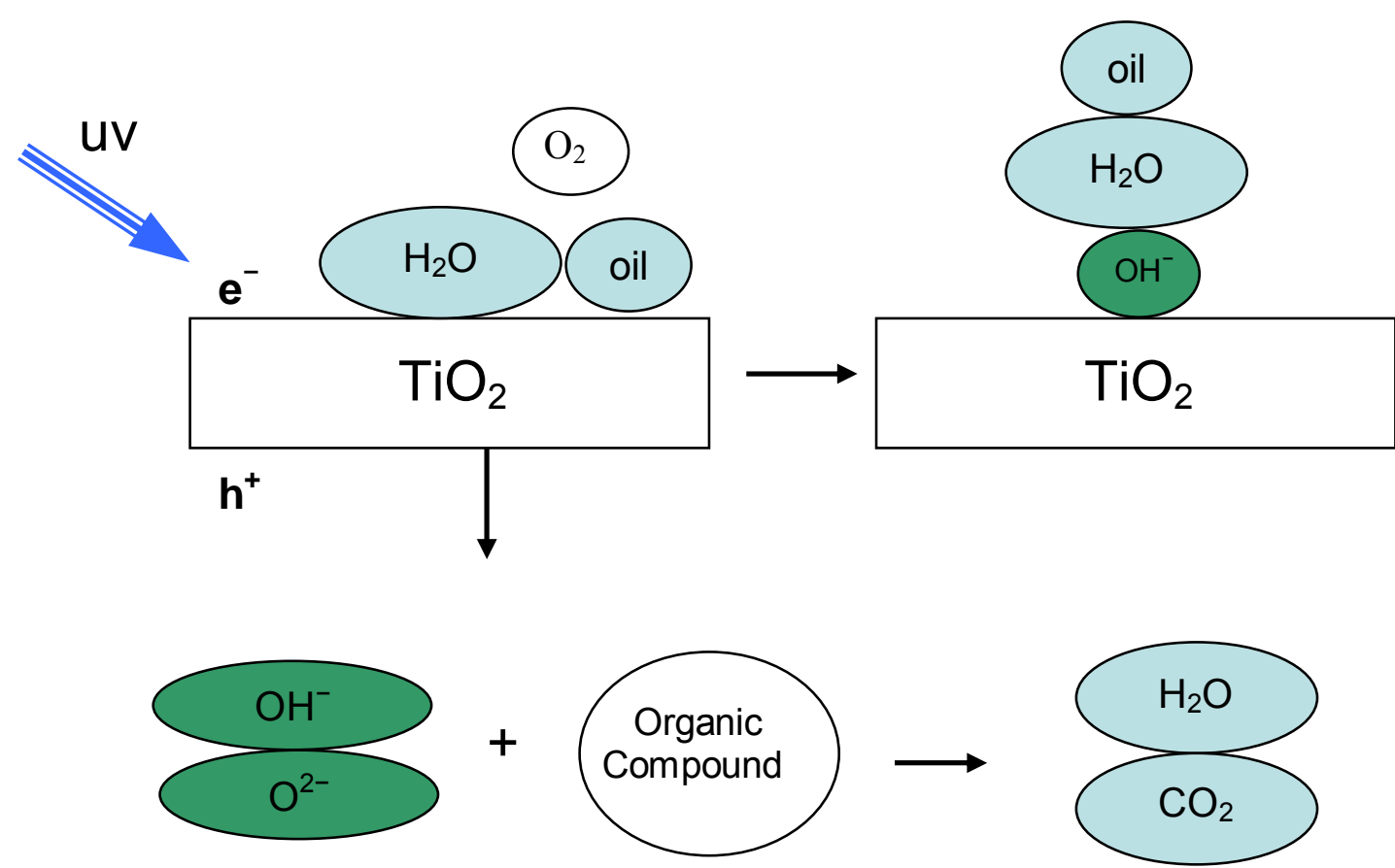

Figure 1.6. A schematic illustration of the self-cleaning and self-sterilization properties of $\mathrm{TiO}_{2}$. 


$$
\mathrm{H}_{2} \mathrm{O}_{2}+\mathrm{e}^{-} \rightarrow \mathrm{OH}^{-}+\cdot \mathrm{OH}
$$

The hydroxyl radicals produced by $\mathrm{TiO}_{2}$ can break the molecular bonds of organic compounds and other biological species. After decomposing, those compounds and biological species change into the simple chemical forms of $\mathrm{CO}_{2}$ and $\mathrm{H}_{2} \mathrm{O}$. Thus, in the presence of uv light, $\mathrm{TiO}_{2}$ can destroy bacteria, viruses, and dust mites in air. The hydroxyl radicals can bond to the surface of $\mathrm{TiO}_{2}$ films deposited on the surface of window glass and form an "intermediate zone" separating dust from the glass. The dust can be flushed away by rains. Thus the window glass deposited with $\mathrm{TiO}_{2}$ thin film is capable of self-cleaning, and the cleaning cycles of windows in tall buildings can be greatly reduced. Figure 1.6 simply illustrates the mechanism of how $\mathrm{TiO}_{2}$ works as a self-cleaning and self-sterilizing photocatalyst. 


\section{Chapter 2}

\section{Experimental Techniques}

\subsection{Introduction}

Electron paramagnetic resonance (EPR), also known as electron spin resonance (ESR), is a spectroscopic technique used to detect unpaired electrons in materials. Soviet physicist Yevgeny Zavoisky first experimentally observed an EPR signal in 1944. Since then, significant advances have been made in the electronics and the computer data acquisition techniques. These improvements in instrumentation contribute to the popularity of the EPR technique, primarily because spectrometers today are more integrated and easier to operate. At the present time, EPR is widely used technique in physics (especially solid state physics), chemistry, geology, biology, biochemistry, and medicine.

EPR is highly sensitive, with the minimum detectable number of electron spins in the $10^{10}$ range. For typical sample volumes, this translates into the ability to detect concentrations of paramagnetic point defects on the order of a few ppb (i.e., near $10^{13} \mathrm{~cm}^{-3}$ ). EPR does not have special requirements for the sample, in other words, samples used in EPR study can be solid, liquid, or gas. EPR is nondestructive, samples will not suffer any physical or chemical damage during EPR measurements. In some investigations, irradiation with $\mathrm{x}$ rays or illumination with laser light may be used to change the charge states of point defects. This converts defects into paramagnetic charge states, and thus makes them "visible" in the EPR experiments. These light-induced changes may be temporary, i.e., the samples recover to their original state in as little as several minutes (in rare case, days or longer) after removing the light.

EPR has proven to be an extremely powerful technique in studying defects in solid state materials, especially in single crystals. EPR can often unambiguously identify the point defects and estimate their concentration. Electron-nuclear double resonance (ENDOR) combines the high sensitivity of nuclear magnetic resonance (NMR) with the 
EPR technique and expands the characterization ability of EPR.

\subsection{Point Defects}

Point defects are localized defects that typically occupy one or two lattice sites in an otherwise perfect crystal lattice. The point defects are usually randomly distributed in a crystal. In the case of unwanted point defects, their concentration may be controlled by modifying the growth. Point defects, however, always exist in every crystal, either as a result of thermodynamics (i.e., minimization of free energy), impure starting materials, or volatility and/or contamination during growth. Therefore, it is important to study point defects and understand their potential affects on device performance.

Some point defects are unintentionally introduced into crystals during growth and thus cause the crystals to exhibit unwanted behaviors. For example, $\mathrm{Ti}^{3+}$ ions in KTP crystals are suggested to be the origin of the well known gray tracks, which limit the performance of the crystal in high power and high energy density laser applications [2.1]. Other point defects can improve the performance of a crystal, and thus are intentionally added into crystals either by doping during growth or by post-growth treatments. For example, rare-earth ions are often doped into different materials to produce high-power solid-state lasers. One of the better known laser-host materials is Nd:YAG.

In general, point defects can be separated into two basic types.

1. Intrinsic (or native) point defects: These include vacancies, interstitials, and antisites.

Vacancies: an atom is absent from its site in the regular lattice.

Interstitials: an atom occupies a site that is not part of the regular lattice.

Antisites: an atom occupies a site in the regular lattice that is normally occupied by a different type of atom.

2. Extrinsic point defects: These are impurities. Examples are $\mathrm{Fe}^{3+}$ and $\mathrm{Cr}^{3+}$ ions substituting for $\mathrm{Ti}^{4+}$ ions in $\mathrm{TiO}_{2}$ crystals.

Point defects often have unpaired electrons, or spins, and thus are detectable by EPR 
spectrometers. If a point defect does not have unpaired spins, irradiation with $\mathrm{x}$ rays or laser light can change the charge state of a point defect and thus make it paramagnetic. For example, the $\mathrm{Ti}^{3+}$ center labeled $\mathrm{A}_{\text {flux }}$ in flux-grown $\mathrm{KTiOPO}_{4}$ crystals was produced by an irradiation with $\mathrm{x}$ rays [2.1].

EPR experiments can provide a wealth of useful information about point defects. 1. Characterizing a point defect by analyzing its hyperfine patterns. Point defects, such as transition-metal ions and rare-earth ions (e.g., $\mathrm{Er}^{3+}, \mathrm{Cr}^{3+}$, etc.), usually have a non-zero nuclear spin in addition to unpaired electron spin(s). Also, neighboring atoms may have non-zero nuclear spins. The hyperfine interactions between the unpaired electron spin(s) and these nuclear spins often provide unique information about the nature of the unpaired electrons' wave function and the relative positions of the nearby atoms. Therefore, the point defects can often be unambiguously indentified.

2. Monitoring changes in the charge state of a point defect. Laser and $\mathrm{x}$-ray irradiations can change the charge state of a point defect from nonparamagnetic to paramagnetic or vice versa. Comparing an EPR spectrum before and after an irradiation allows specific charge states of a point defect to be monitored.

3. Determining the concentration of a point defect. The relative concentrations of point defects can be determined by comparing the EPR spectra taken under identical conditions from different samples of the same material. Also, comparing the EPR spectrum of a defect before and after a modifying treatment (thermal anneals, irradiations, etc.) allows relative changes in the defect's concentration to be monitored [2.2]. It is also possible to make an estimate of the absolute concentration of a point defect using EPR. The following empirical equation has proven to be useful for determining the concentration, $\mathrm{N}$, of an EPR-active point defect.

$$
\mathrm{N}=\left(5 \times 10^{10} \text { spins }\right)(\Delta \mathrm{W})^{2}(\mathrm{~S} / \mathrm{N})(\# \text { of lines })(\mathrm{T} / 10)(1 / \mathrm{V})
$$

Here, $5 \times 10^{10}$ is the minimum number of spins the system can detect at $10 \mathrm{~K}, \Delta \mathrm{W}$ is the linewidth of the EPR signal in gauss, $\mathrm{S} / \mathrm{N}$ is the signal-to-noise ratio, $\mathrm{T}$ is the temperature 
of the sample in $\mathrm{K}$, and $\mathrm{V}$ is the volume of the sample in $\mathrm{cm}^{3}$.

4. Determining the symmetry of a point defect. The angular dependence of an EPR spectrum reflects the symmetry of the point defect and provides a complete set of spinHamiltonian parameters. A structural model of the point defect can be established once these spin-Hamiltonian parameters are known.

\subsection{Basic Principles of EPR}

A free electron produces a small magnetic field due to its intrinsic spin (electron spin) and acts like a bar magnet. When exposed to a strong external magnetic field $\mathbf{B}$, this "bar magnet" aligns either along or against B. An electron spin aligning along $\mathbf{B}$ is referred to as the "spin-up" state, while an electron spin aligning against $\mathbf{B}$ is referred to as the "spin-down" state. The spin-up state has a lower energy than the spin-down state. An electron can "flip" from the spin-up state to the spin-down state by absorbing a photon of the appropriate energy, or from the spin-down to the spin-up state by emitting a photon. The energy of the absorbed or emitted photons must be equal to the energy difference between the spin-up and spin-down states. This transition from one state to the other by absorbing a photon is referred to as resonance. The Stern-Gerlach experiment demonstrated that electrons have an intrinsic magnetic moment.

Classically, when a magnetic moment $\mu$ is placed in an external magnetic field B, the energy of this moment is

$$
\mathrm{E}=\boldsymbol{\mu} \cdot \mathbf{B} .
$$

Quantum mechanically, a free electron will have a magnetic moment

$$
\mu=-\beta g_{\mathrm{e}} \mathbf{S}
$$

where $\beta=\mathrm{eh} / 2 \mathrm{mc}$ is the Bohr magneton, $\mathrm{g}_{\mathrm{e}}=2.0023$ is the free electron $\mathrm{g}$ value, and $\mathrm{S}$ is the electron spin operator. Thus, the energy of an electron in a magnetic field $\mathbf{B}$ will be

$$
\mathbf{H}=\boldsymbol{\mu} \cdot \mathbf{B}=-\beta \mathrm{g}_{\mathrm{e}} \mathbf{S} \cdot \mathbf{B} .
$$

Let the direction of the magnetic field be $\mathrm{z}$, then $\mathrm{B}_{\mathrm{x}}=\mathrm{B}_{\mathrm{y}}=0$, and the Hamiltonian is 


$$
\mathbf{H}=-\beta g_{\mathrm{e}} \mathrm{S} \cdot \mathbf{B}=-\beta \mathrm{g}_{\mathrm{e}} \mathrm{S}_{\mathrm{z}} \mathrm{B}_{\mathrm{z}}
$$

A free electron has spin $S=1 / 2$, with eigenvalues of $m_{S}=1 / 2$ and $m_{S}=-1 / 2$. Thus, the two energy states of the electron are

$$
\mathrm{E}= \pm 1 / 2 \beta \mathrm{g}_{\mathrm{e}} \mathrm{B}
$$

The energy difference between these two states is $\Delta \mathrm{E}=\beta \mathrm{g}_{\mathrm{e}} \mathrm{B}$, which is proportional to the magnitude of the external magnetic field B. The energy of the ground state (spin-up) of a free electron in an external magnetic field $\mathbf{B}$ is $\mathrm{E}=-1 / 2 \beta \mathrm{g}_{\mathrm{e}} \mathrm{B}$. By absorbing a photon with energy $h v=\Delta E=\beta g_{\mathrm{e}} \mathrm{B}$, the electron flips to the higher energy state (spin-down).

In an X-band EPR spectrometer, the photon source operates at a microwave frequency $v$ near $9.5 \mathrm{GHz}$. The energy difference between the two spin states is tuned by changing the magnitude of magnetic filed $\mathbf{B}$. A resonance occurs when $h v=\Delta \mathrm{E}=\beta \mathrm{g}_{\mathrm{e}} \mathrm{Br}$, where $\mathrm{Br}$ is the field at resonance.

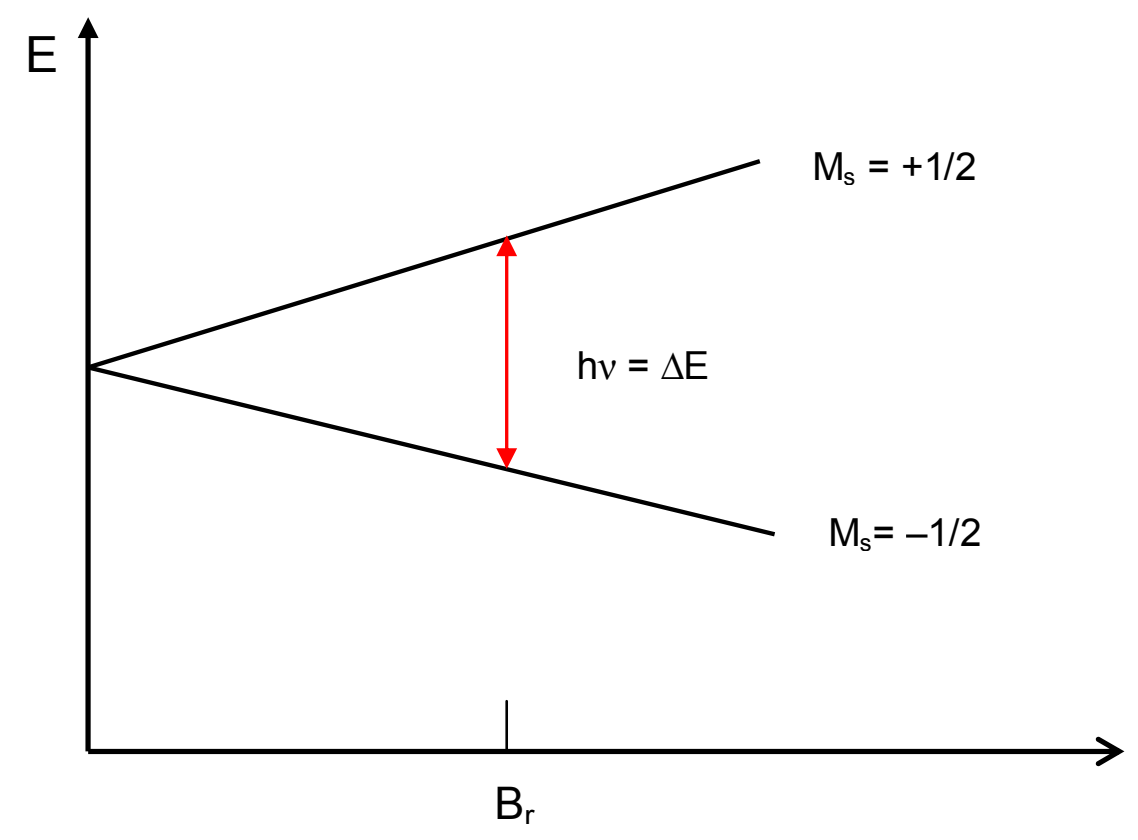

Figure 2.1. A schematic illustration of the EPR transition for a spin $\mathrm{S}=1 / 2$. 


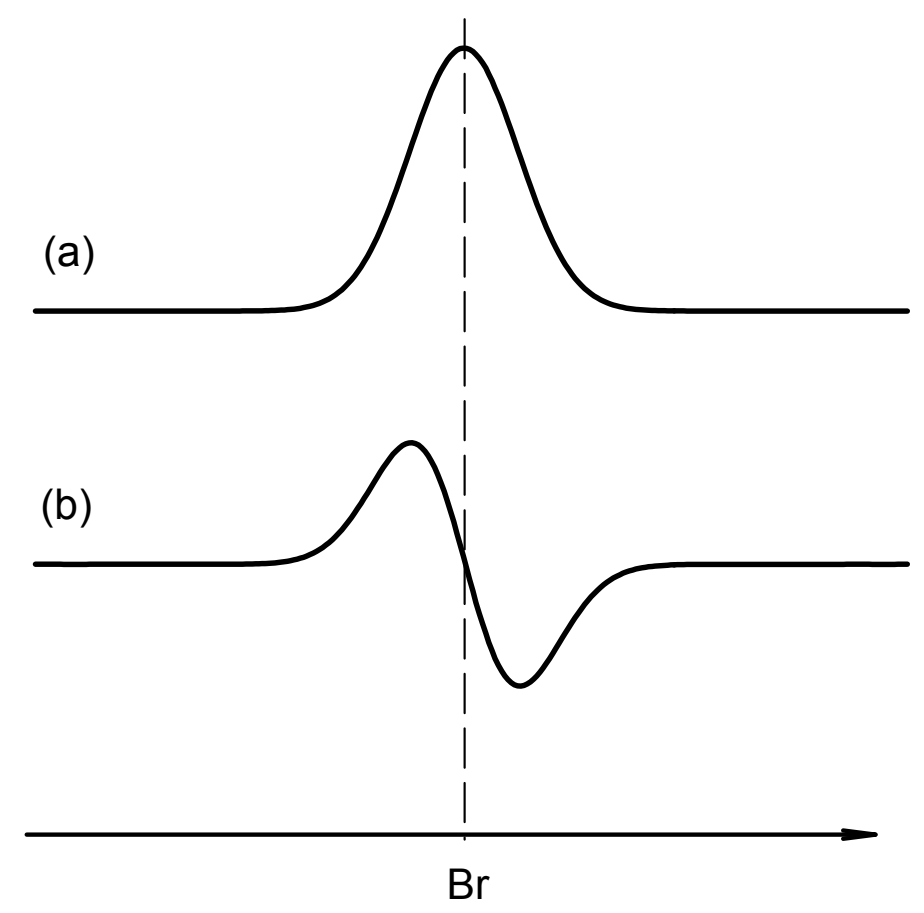

Figure 2.2. (a) Absorption signal representing an EPR resonance for a spin $\mathrm{S}=$ 1/2. (b) The first derivative of the absorption signal, which represents the EPR signal.

Figure 2.2(a) shows the absorption signal corresponding to an EPR transition at the resonance field Br. In an EPR spectrometer, the recorded EPR signals do not appear as absorption signals. Because the concentration of paramagnetic defects may be very small (on the order of a few ppm or less), the absorption signal will be very weak and be "buried" within the noise. Phase-sensitive detection techniques are employed in EPR spectrometers to increase the signal-to-noise ratio. A $100 \mathrm{kHz}$ reference oscillator is used to modulate the static magnetic field and the resulting EPR signal appears as the first derivative of the absorption signal, as shown in Fig. 2.2(b). This use of phase-sensitive detection increases the sensitivity of the EPR spectrometer by many orders of magnitude. More information about phase-sensitive detection can be found in references [2.3] and [2.4]. 


\subsection{Hyperfine Interactions}

Hyperfine interactions occur when the magnetic moment of a nucleus interacts with the magnetic moment of an unpaired electron. Interactions between one electron magnetic moment and another electron magnetic moment are referred to as fine structure. Most of the electrons in insulators and semiconductors are part of closed shells, and thus are paired. If the unpaired electrons (spins) are isolated, they will give rise to an EPR spectrum when the sample is taken to an appropriate temperature. The unpaired spin may be influenced by other nearby unpaired electron spins, other surrounding nuclei, or an internal electric field (i.e., the crystal field). The hyperfine interaction is often described by a hyperfine matrix $\mathbf{A}$.

For simplicity, consider a spin $(\mathbf{S})$ that interacts with a nearby nucleus with a

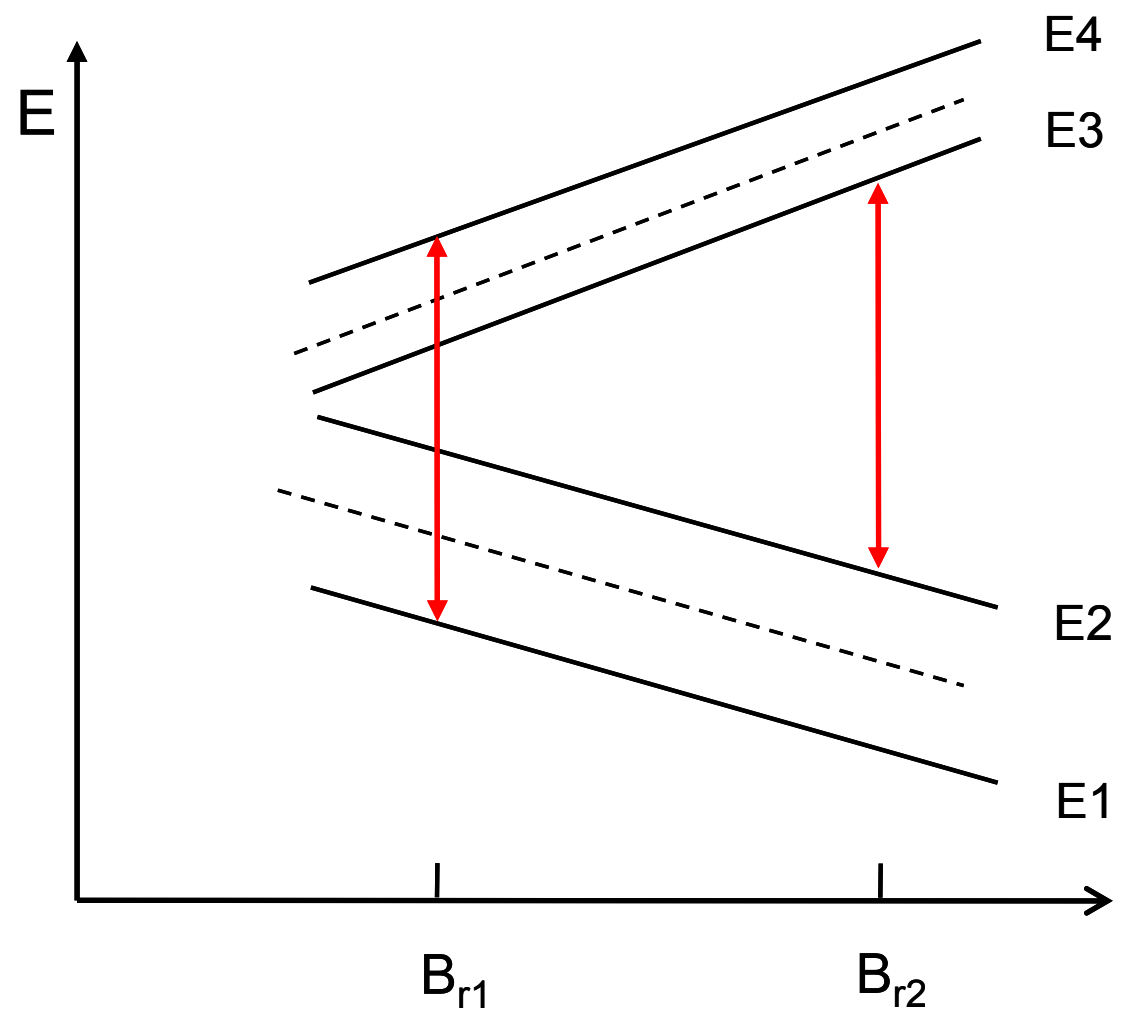

Figure 2.3. A schematic illustration of the EPR transitions for a $\mathrm{S}=1 / 2$ and $\mathrm{I}=$ $1 / 2$ spin system. 
nuclear moment (I). The resulting spin Hamiltonian is

$$
\mathbf{H}=\beta \mathbf{S} \cdot \mathbf{g} \cdot \mathbf{B}+\mathbf{I} \cdot \mathbf{A} \cdot \mathbf{S}-\mathrm{g}_{\mathrm{N}} \beta_{\mathrm{N}} \mathbf{I} \cdot \mathbf{B} .
$$

Here, $\beta \mathbf{S} \cdot \mathbf{g} \cdot \mathbf{B}$ is the electron Zeeman term, $\mathbf{I} \cdot \mathbf{A} \cdot \mathbf{S}$ is the hyperfine term, and $-\mathrm{g}_{\mathrm{N}} \beta_{\mathrm{N}} \mathbf{I}$ - $\mathbf{B}$ is the nuclear Zeeman term. In crystalline materials, the g value and the hyperfine constant $\mathrm{A}$ of a point defect are often matrices and exhibit angular dependences. Anisotropy in the g matrix is the result of the interaction between the electron spin and the orbital angular momentum. The following is a simple case in which $g$ and A are isotropic, and the principal axes of the g matrix and A matrix are the same as the crystal axes.

Let the direction of the external magnetic field be the $\mathrm{z}$ direction, then

$$
\mathbf{H}=\beta \mathbf{S} \cdot \mathbf{g} \cdot \mathbf{B}+\mathbf{I} \cdot \mathbf{A} \cdot \mathbf{S}-\mathrm{g}_{\mathrm{N}} \beta_{\mathrm{N}} \mathbf{I} \cdot \mathbf{B}
$$

and

$$
\begin{aligned}
\mathrm{E}\left(\mathrm{M}_{\mathrm{S}}, \mathrm{M}_{\mathrm{I}}\right) & =\beta \mathrm{gBM}_{\mathrm{S}}+\mathrm{AMsM}_{\mathrm{I}}-\mathrm{g}_{\mathrm{N}} \beta_{\mathrm{N}} \mathrm{B} \mathrm{M}_{\mathrm{I}} \\
& =\beta g \mathrm{gBM}_{\mathrm{S}}+\mathrm{M}_{\mathrm{I}}\left(\mathrm{AM}_{\mathrm{S}}-\mathrm{g}_{\mathrm{N}} \beta_{\mathrm{N}} \mathrm{B}\right) \\
& =\beta g \mathrm{gBM}_{\mathrm{S}}+\mathrm{M}_{\mathrm{I}}\left(\mathrm{AM}_{\mathrm{S}}-v_{\mathrm{N}}\right)
\end{aligned}
$$

When $\mathrm{S}=1 / 2$ and $\mathrm{I}=1 / 2$, then $\mathrm{M}_{\mathrm{S}}= \pm 1 / 2, \mathrm{M}_{\mathrm{I}}= \pm 1 / 2$, the four energy levels are

$$
\begin{array}{ll}
\mathrm{E} 1=-\beta \mathrm{gB} / 2-\left(\mathrm{A} / 2+v_{\mathrm{N}}\right) / 2, & \left(\mathrm{M}_{\mathrm{S}}=-1 / 2, \mathrm{M}_{\mathrm{I}}=1 / 2\right) \\
\mathrm{E} 2=-\beta \mathrm{gB} / 2+\left(\mathrm{A} / 2+v_{\mathrm{N}}\right) / 2, & \left(\mathrm{M}_{\mathrm{S}}=-1 / 2, \mathrm{M}_{\mathrm{I}}=-1 / 2\right) \\
\mathrm{E} 3=\beta \mathrm{gB} / 2-\left(\mathrm{A} / 2-v_{\mathrm{N}}\right) / 2, & \left(\mathrm{M}_{\mathrm{S}}=1 / 2, \mathrm{M}_{\mathrm{I}}=-1 / 2\right) \\
\mathrm{E} 4=\beta \mathrm{gB} / 2+\left(\mathrm{A} / 2-v_{\mathrm{N}}\right) / 2, & \left(\mathrm{M}_{\mathrm{S}}=1 / 2, \mathrm{M}_{\mathrm{I}}=1 / 2\right)
\end{array}
$$

For $\mathrm{A} / 2>v_{\mathrm{N}}$, one has E4 $>\mathrm{E} 3>\mathrm{E} 2>\mathrm{E} 1$, which corresponds to the energy level versus magnetic field diagram that is shown in Fig. 2.3. When microwave photons with the appropriate energy are present while the magnetic field is being swept, resonances will occur at $\mathrm{B}_{\mathrm{r} 1}$ and $\mathrm{B}_{\mathrm{r} 2}$. The electron transitions from $\mathrm{E} 1$ to $\mathrm{E} 4$ and from $\mathrm{E} 2$ to $\mathrm{E} 3$ occur at $\mathrm{B}_{\mathrm{r} 1}$ and $\mathrm{B}_{\mathrm{r} 2}$, respectively. These two transitions are referred to as "allowed" and they obey the following selection rules governing EPR transitions $\left(\Delta \mathrm{Ms}= \pm 1\right.$ and $\left.\Delta \mathrm{M}_{\mathrm{I}}=0\right)$. In contrast, the transitions from E2 to E4 and E1 to E3 are referred to as "forbidden" EPR 
transitions where $\Delta \mathrm{Ms}= \pm 1$ and $\Delta \mathrm{M}_{\mathrm{I}}= \pm 1$.

In the case of $\mathrm{A} / 2<v_{\mathrm{N}}$, one has $\mathrm{E} 3>\mathrm{E} 4>\mathrm{E} 2>\mathrm{E} 1$, but the transitions from E1 to E4 and E2 to E3 are still the allowed transitions. In either case, $A / 2<v_{N}$ or $A / 2>v_{N}$, one obtains:

$$
\begin{aligned}
& \mathrm{E} 4-\mathrm{E} 1=\beta \mathrm{gB}+\mathrm{A} / 2 \\
& \mathrm{E} 3-\mathrm{E} 2=\beta \mathrm{gB}-\mathrm{A} / 2
\end{aligned}
$$

Assuming the photon energy is $h v$, the resonance fields $\mathrm{B}_{\mathrm{r} 1}$ and $\mathrm{B}_{\mathrm{r} 2}$ are given by

$$
\mathrm{E} 4\left(\mathrm{~B}_{\mathrm{r} 1}\right)-\mathrm{E} 1\left(\mathrm{~B}_{\mathrm{r} 1}\right)=\beta \mathrm{gB} \mathrm{B}_{\mathrm{r} 1}+\mathrm{A} / 2=h v
$$

and

$$
\mathrm{E} 3\left(\mathrm{~B}_{\mathrm{r} 2}\right)-\mathrm{E} 2\left(\mathrm{~B}_{\mathrm{r} 2}\right)=\beta \mathrm{gB} \mathrm{B}_{\mathrm{r} 2}-\mathrm{A} / 2=h v
$$

Combining these last two equations leads to

$$
\mathrm{B}_{\mathrm{r} 2}-\mathrm{B}_{\mathrm{r} 1}=\mathrm{A} / \beta \mathrm{g} .
$$

The units of $\mathrm{A}$ are $\mathrm{MHz}$. The term $\mathrm{A} / \beta \mathrm{g}$ equals the separation between the two allowed EPR lines. $\mathrm{A} / \beta \mathrm{g}$ is often simply noted as $\mathrm{A}$ in units of Gauss (as shown in Fig. 2.4).

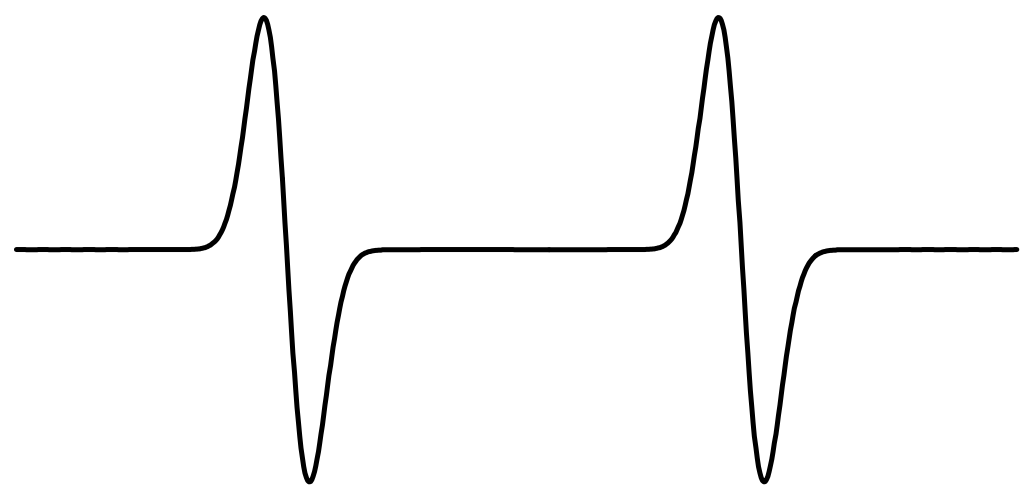

A

Figure 2.4. A simulated hyperfine spectrum for a $S=1 / 2$ and $I=1 / 2$ spin system. 


\subsection{Electron-Nuclear Double Resonance}

Well-resolved hyperfine splittings in EPR spectra may provide information about the responsible nuclei. In those cases where more than one contributing nucleus has the same nuclear spin, it is difficult to identify the responsible nucleus using only the EPR spectrum. For example, hydrogen $(\mathrm{H})$ and phosphors $(\mathrm{P})$ nuclei both have $\mathrm{I}=1 / 2$. In addition, in a case where the electron spin only weakly interacts with the involved nuclear moments, the hyperfine splitting could be smaller than the linewidth of the EPR signals. This might lead to misinterpretations. Electron-nuclear double resonance (ENDOR) is capable of removing the ambiguity in the two cases described above.

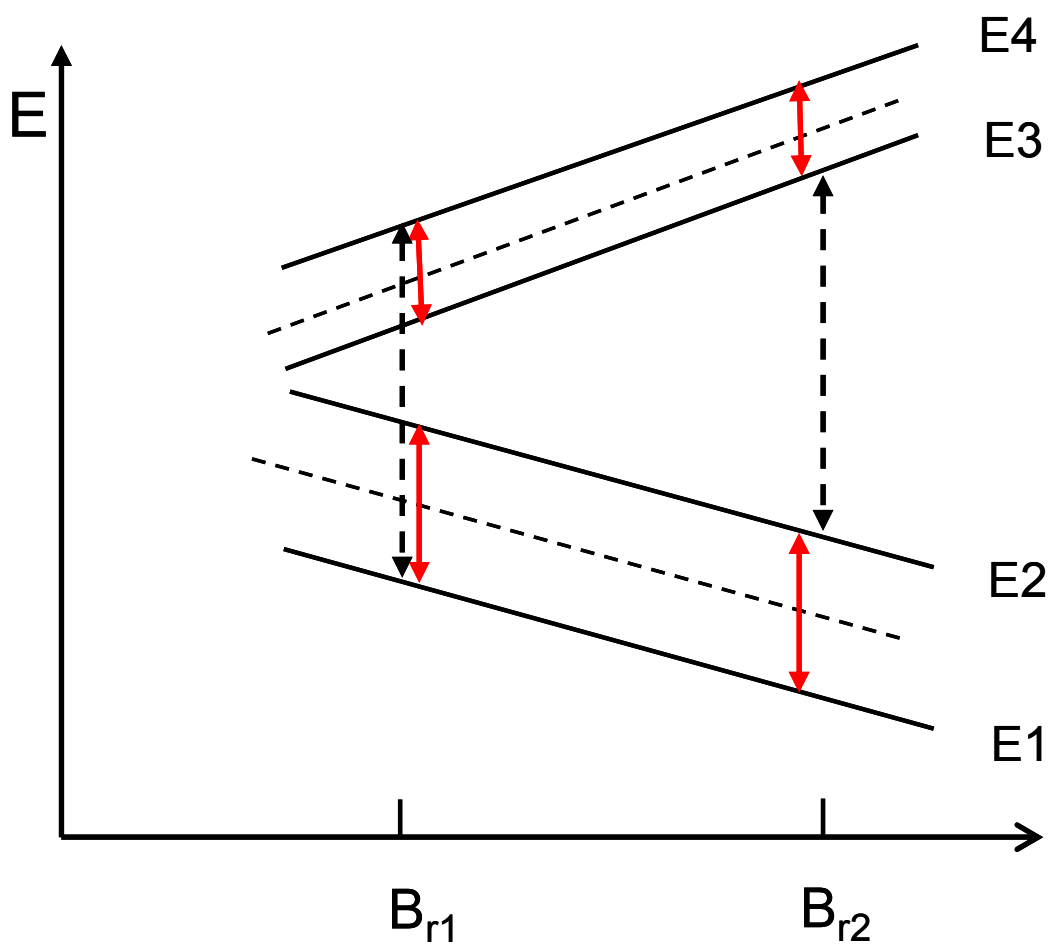

Figure 2.5. A schematic illustration of the ENDOR transitions for a $\mathrm{S}=1 / 2, \mathrm{I}=$ $1 / 2$ spin system. The red solid lines show the ENDOR transitions. The black dashed lines show the EPR transitions. 


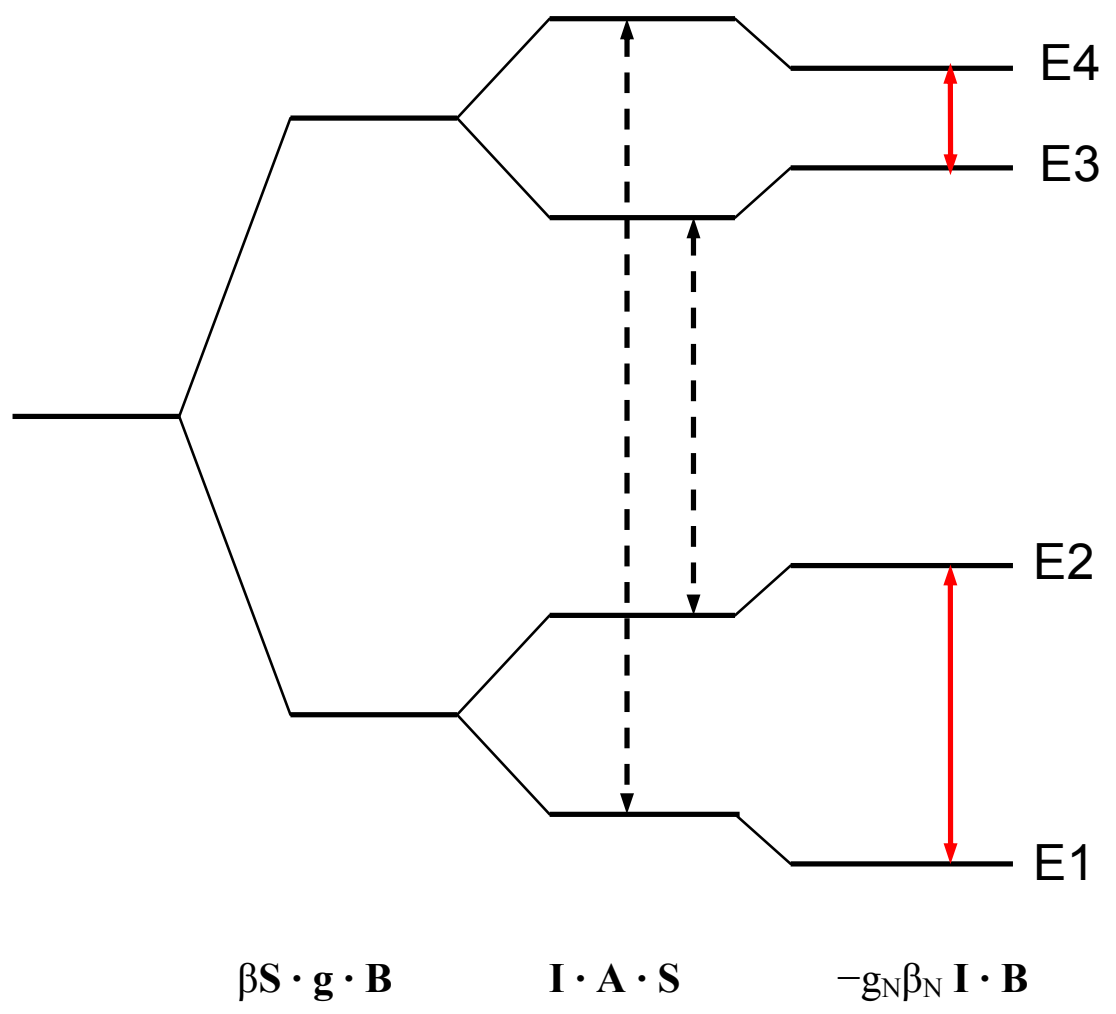

Figure 2.6. A schematic illustration of the energy splittings for a $\mathrm{S}=1 / 2$ and $\mathrm{I}=$ $1 / 2$ spin system when placed in a fixed external magnetic field.

For simplicity and clarity as before, consider the case $\mathrm{S}=1 / 2$ and $\mathrm{I}=1 / 2$, and assume $\mathrm{A} / 2>v_{\mathrm{N}}$. Figure 2.5 illustrates the energy-level splitting of the system versus the external magnetic field. The two dashed arrows represent the two allowed EPR transitions described earlier. The two red solid arrows indicate the additional transitions E1 to E2 and E3 to E4. These latter two transitions are referred to as allowed ENDOR transitions and are determined by the selection rules governing ENDOR transitions $\left(\Delta \mathrm{M}_{\mathrm{I}}= \pm 1\right.$ and $\left.\Delta \mathrm{M}_{\mathrm{S}}=0\right)$.

Figure 2.6 shows the energy splitting of the spin system in a fixed external magnetic field. A Hamiltonian with the following form is considered.

$$
\mathbf{H}=\beta \mathbf{S} \cdot \mathbf{g} \cdot \mathbf{B}+\mathbf{I} \cdot \mathbf{A} \cdot \mathbf{S}-\mathrm{g}_{\mathrm{N}} \beta_{\mathrm{N}} \mathbf{I} \cdot \mathbf{B}
$$


The first term is the electron Zeeman term which results in the largest energy splitting. The second term is the hyperfine interaction and the third one is the nuclear Zeeman term. Assuming A/2 $>v_{\mathrm{N}}$, the dashed lines indicate the allowed EPR transitions (from E1 to E4 and E2 to E3), and the solid lines (red) indicate the allowed ENDOR transitions (from E1 to E2 and E3 to E4).

\subsection{EPR and ENDOR Instruments}

Two Bruker electron paramagnetic resonance systems (including a Bruker EMX EPR spectrometer and a Bruker Elexsys E-500 ENDOR spectrometer) were used in this dissertation research. The two spectrometers operate near a microwave frequency of 9.50 GHz. The EMX system has a $\mathrm{TE}_{102}$ rectangular microwave cavity, while the Elexsys system has a cylindrical microwave cavity. In an ENDOR experiment, a RF frequency field is required to flip the nuclear spin. The conducting helical ENDOR coil mounted with its axis vertical in the cavity perturbs a cylindrical cavity less than a rectangular one. More details about the EPR and ENDOR instruments can be found in references [2.5] and $[2.6]$

Oxford helium-gas-flow systems were used to maintain the sample temperature in the 4 to $40 \mathrm{~K}$ range. The static magnetic field was measured using proton NMR gaussmeters. A small Cr-doped $\mathrm{MgO}$ crystal was used to correct for the difference in magnetic field between the sample and the probe tips of the gaussmeters (the isotropic $g$ value for $\mathrm{Cr}^{3+}$ in $\mathrm{MgO}$ is 1.9800$)$. A Hewlett Packard frequency counter was used to measure the microwave frequency during the EPR and ENDOR experiments. In practice, the magnetic field values of individual lines in an EPR spectrum are measured using the gaussmeter. Then the sample is taken out without moving the cavity and the $\mathrm{MgO}: \mathrm{Cr}$ crystal is placed where the sample was originally located The following equation is used for the magnetic field corrections. 


$$
\begin{aligned}
\mathrm{B}_{\text {corrected }} & =\frac{h v_{C r}}{g_{C r} \beta B_{C r}} B_{\text {measured }} \\
& =\frac{360.847 \times v_{C r}}{B_{C r}} B_{\text {measured }}
\end{aligned}
$$

Here, $B_{\text {measured }}$ is the magnetic field value corresponding to a particular EPR line in the spectrum taken from the EPR spectrum using the gaussmeter. $B_{C r}$ and $v_{C r}$ are the field value and the frequency of $\mathrm{Cr}^{3+} \mathrm{EPR}$ signal.

A He-Cd laser capable of providing two different wavelengths of laser light (i.e., 442 and $325 \mathrm{~nm}$ ) was used as an illumination source for the photoinduced EPR studies. Narrow slots in the end of the Bruker $\mathrm{TE}_{102}$ rectangular microwave cavity allowed optical access to the sample. Approximately $15 \mathrm{~mW}$ of $442 \mathrm{~nm}$ light (or $4 \mathrm{~mW}$ of $325 \mathrm{~nm}$ light) from the He-Cd laser was incident on the sample during illuminations at low-temperature. 


\section{Chapter 3}

\section{Previous EPR Studies on $\mathrm{TiO}_{2}$ Crystals}

\subsection{Introduction}

In Chapter 1, I pointed out that electron paramagnetic resonance has been used to study point defects in $\mathrm{TiO}_{2}$ (rutile) crystals for over five decades. Most of these studies are focused on reduced crystals or intentionally doped crystals. In this chapter, I give a brief review of previous EPR studies on $\mathrm{TiO}_{2}$ (rutile) single crystals. Generally, there are two types of paramagnetic centers in $\mathrm{TiO}_{2}$ (rutile): native defects and impurities. The most likely native defects are oxygen vacancies and interstitial titanium ions. Although it is possible that they exist in crystals, titanium vacancies are not expected because of their high formation energy. Common impurities include the transition-metal ions $\mathrm{Fe}^{3+}$ and $\mathrm{Cr}^{3+}$. Many other impurities can exist in the $\mathrm{TiO}_{2}$ lattice as a result of intentional doping or unintentional contamination.

\section{2. $\mathrm{Fe}^{3+}$ and $\mathrm{Cr}^{3+}$ Impurities}

Transition-metal ions in $\mathrm{TiO}_{2}$ can be easily and unambiguously identified because of their unique hyperfine patterns. Fe and $\mathrm{Cr}$ are common impurities in many materials, and also are the most common impurities in $\mathrm{TiO}_{2}$ crystals. In the course of my research, I have studied more than 20 different single crystals of $\mathrm{TiO}_{2}$ obtained from four different sources, and each of them has been found to contain significant concentrations of Fe and Cr impurities. These concentrations were observed to vary from sample to sample. Even though the EPR signals of $\mathrm{Fe}^{3+}$ and $\mathrm{Cr}^{3+}$ can be observed up to room temperature, better resolved details associated with their hyperfine interaction can be obtained from low temperature EPR experiments. Figure 3.1 shows an EPR spectrum containing signals from $\mathrm{Fe}^{3+}$ and $\mathrm{Cr}^{3+}$ ions in $\mathrm{TiO}_{2}$ crystals. These data were taken at $30 \mathrm{~K}$ with the magnetic field parallel to the [001] direction in the crystal. The EPR line near $843 \mathrm{G}$ is 


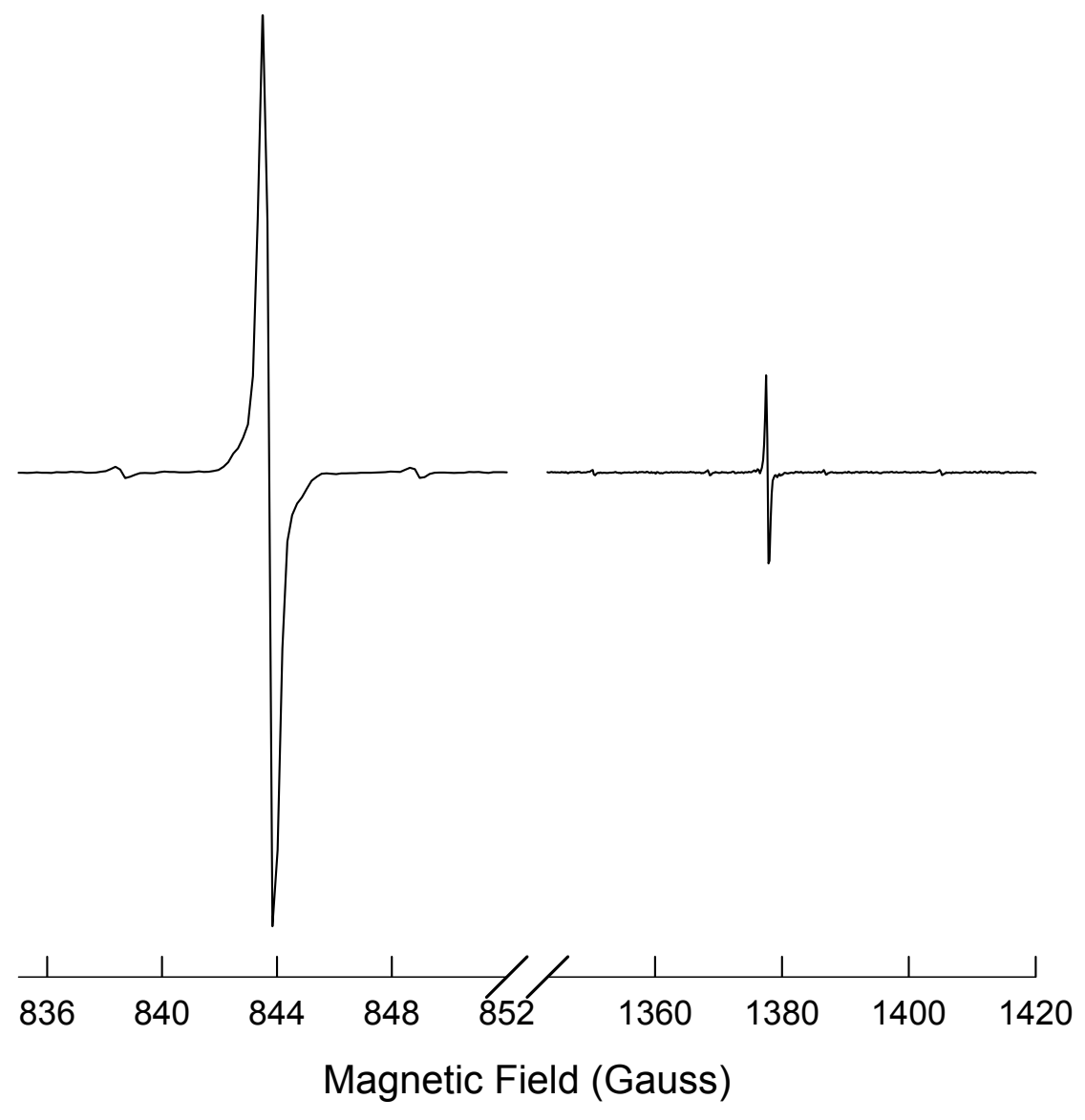

Figure 3.1. An EPR spectrum of $\mathrm{Cr}^{3+}$ and $\mathrm{Fe}^{3+}$ ions in a $\mathrm{TiO}_{2}$ crystal. These data were taken at $30 \mathrm{~K}$ with the magnetic field along the [001] direction. The microwave frequency was near $9.59 \mathrm{GHz}$.

due to $\mathrm{Fe}^{3}$, while the EPR line near $1378 \mathrm{G}$ is due to $\mathrm{Cr}^{3+}$. The effective $\mathrm{g}$ values for these two EPR centers are $\mathrm{g}_{[001]}=8.10\left(\right.$ for $\left.\mathrm{Fe}^{3+}\right)$ and $\mathrm{g}_{[001]}=4.96\left(\right.$ for $\left.\mathrm{Cr}^{3+}\right)$. These trivalent $\mathrm{Fe}^{3+}$ and $\mathrm{Cr}^{3+}$ ions substitute for $\mathrm{Ti}^{4+}$ ions and provide charge compensation for remote oxygen vacancies $[3.1,3.2]$.

\section{3. $\mathrm{Cu}^{2+}$ Impurities}

Copper is another common impurity in $\mathrm{TiO}_{2}$ that appears in every sample in my study. In some of the samples, it has a low concentration and is difficult to observe. The EPR spectrum of $\mathrm{Cu}^{2+}$ can be easily identified by its hyperfine interaction with the two 

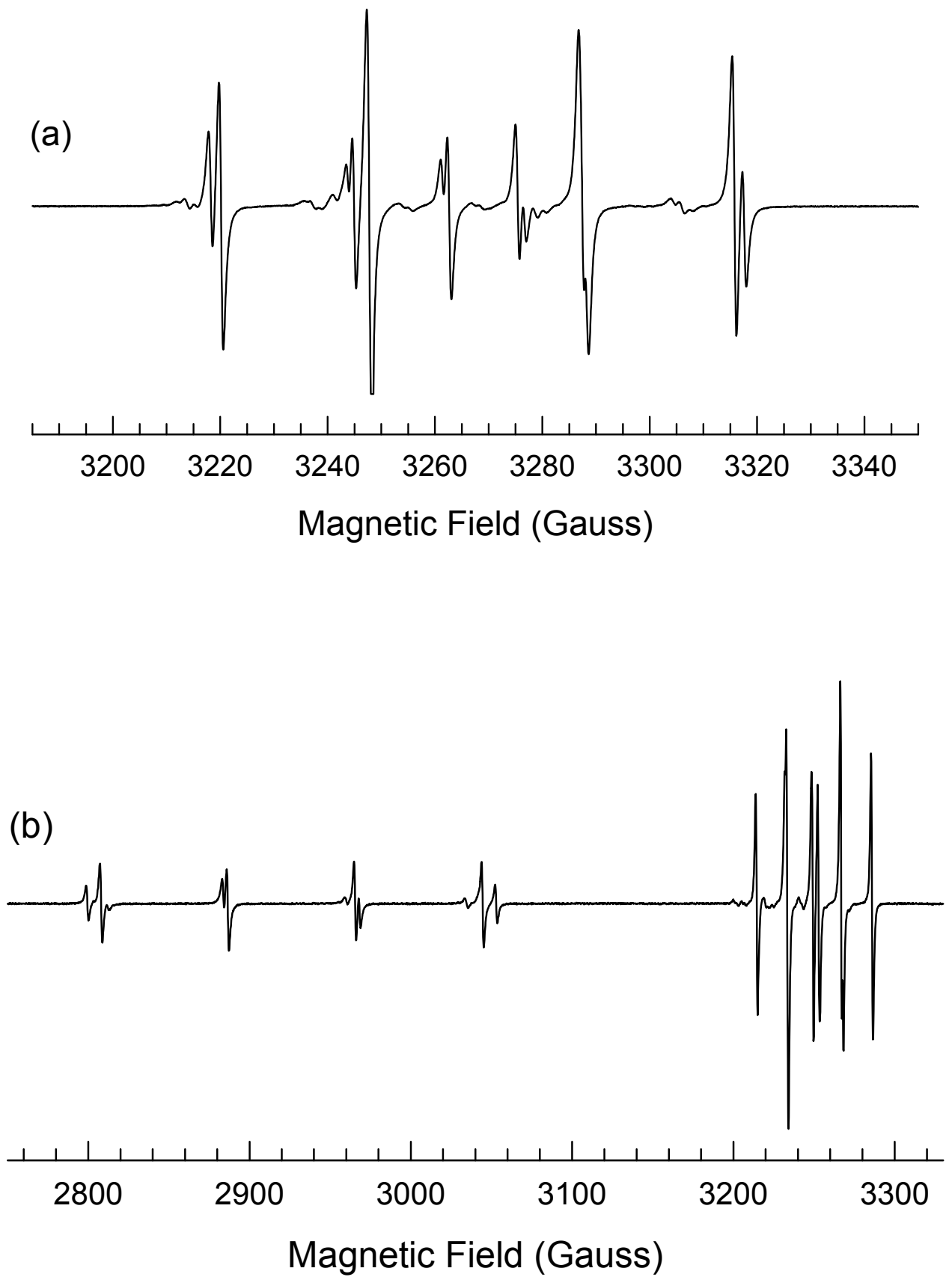

Figure 3.2. EPR spectra of $\mathrm{Cu}^{2+}$ ions in rutile-structured $\mathrm{TiO}_{2}$ crystals. These data were taken at $30 \mathrm{~K}$ with the magnetic field parallel to (a) the [001] direction and (b) the [110] direction. The microwave frequency was near $9.59 \mathrm{GHz}$. 
$\mathrm{Cu}$ nuclei $\left({ }^{63} \mathrm{Cu}\right.$ and $\left.{ }^{65} \mathrm{Cu}\right)$. Gerritsen and Sabisky [3.3] reported the EPR spectrum of $\mathrm{Cu}^{2+}$ in $\mathrm{TiO}_{2}$ (rutile) crystals in 1962, and they suggested that the $\mathrm{Cu}^{2+}$ ions occupied an interstitial site because the radius of a $\mathrm{Cu}^{2+}$ ion $(0.73 \AA)$ is larger than the radius of a $\mathrm{Ti}^{4+}$ ion $(0.60 \AA)$. The spin-Hamiltonian parameters reported by Gerristen and Sabisky are listed in Table 3.1. Later, in 1969, Ensign et al. reported slightly different parameters [3.4]. According to Ensign et al., the $\mathrm{Cu}^{2+}$ ions existing in the $\mathrm{TiO}_{2}$ (rutile) crystals replace substitutional $\mathrm{Ti}^{4+}$ ions.

Table 3.1. Spin-Hamiltonian parameters of $\mathrm{Cu}^{2+}$ in $\mathrm{TiO}_{2}$ (rutile) single crystals. These values are taken from References 3.3 and 3.4.

\begin{tabular}{|c|c|c|c|}
\hline & Gerritsen and Sabisky & \multicolumn{2}{|c|}{ Ensign et al. } \\
\hline $\mathrm{g}_{[110]}$ & 2.105 & \multicolumn{2}{|c|}{2.1084} \\
\hline $\mathrm{g}_{[1-10]}$ & 2.344 & \multicolumn{2}{|c|}{2.3456} \\
\hline $\mathrm{g}_{[001]}$ & 2.093 & \multicolumn{2}{|c|}{2.0935} \\
\hline & & ${ }^{63} \mathrm{Cu}$ & ${ }^{65} \mathrm{Cu}$ \\
\hline $\mathrm{A}_{[110]}(\mathrm{MHz})$ & -57 & 56.1 & 56.4 \\
\hline $\mathrm{A}_{[1-10]}(\mathrm{MHz})$ & -264 & -262.3 & -280.9 \\
\hline $\mathrm{A}_{[001]}(\mathrm{MHz})$ & -87 & 81.5 & 86 \\
\hline $\mathrm{P}_{[110]}(\mathrm{MHz})$ & - & -7.6 & -7.8 \\
\hline $\mathrm{P}_{[1-10]}(\mathrm{MHz})$ & - & 14.8 & 14.8 \\
\hline $\mathrm{P}_{[001]}(\mathrm{MHz})$ & - & -7.2 & -7.0 \\
\hline
\end{tabular}

Figure 3.2 shows EPR spectra of $\mathrm{Cu}^{2+}$ impurity ions in a single crystal of $\mathrm{TiO}_{2}$. These data were taken from the CrysTec sample with dimensions of $2.5 \times 3.0 \times 2.0 \mathrm{~mm}^{3}$. Trace (a) was taken with the magnetic field along the [001] direction in the crystal. Normally, a $\mathrm{Cu}^{2+}$ EPR spectrum has four doublets due to the two isotopes, ${ }^{63} \mathrm{Cu}$ and ${ }^{65} \mathrm{Cu}$. (Each isotope has an $\mathrm{I}=3 / 2$ nuclear spin, which results in four EPR lines. The four lines from each isotope are only slightly different because of their similar nuclear moments). 
I note that there are six groups of lines in trace (a), with the middle two groups of lines being forbidden transitions caused by nuclear electric quadruple interactions. Trace (b) was taken with the magnetic field along the [110] direction in the crystal. Here, the signals fall into two distinct sets of lines (four at lower field and four at higher field). The two groups represent site splitting and result from the two differently oriented octahedra in the $\mathrm{TiO}_{2}$ lattice. These two octahedra are magnetically equivalent when the magnetic field is along the [001] direction in the crystal, and they are magnetically nonequivalent when the magnetic field is along the [110] direction.

\subsection{Impurities at High-Symmetry Sites}

Table 3.2 lists spin-Hamiltonian parameters for several impurities in $\mathrm{TiO}_{2}$ crystals that have been reported in the past literature. Unlike $\mathrm{Fe}$ and $\mathrm{Cr}$, these impurities are

Table 3.2. Spin-Hamiltonian parameters of impurities in $\mathrm{TiO}_{2}$ crystals.

\begin{tabular}{|c|c|c|c|c|c|c|c|}
\hline Impurity & $\mathrm{g}_{[110]}$ & $\mathrm{g}_{[1-10]}$ & $\mathrm{g}_{[001]}$ & $\begin{array}{c}\mathrm{A}_{[110]} \\
(\mathrm{MHz})\end{array}$ & $\begin{array}{c}\mathrm{A}_{[1-10]} \\
(\mathrm{MHz})\end{array}$ & $\begin{array}{c}\mathrm{A}_{[001]} \\
(\mathrm{MHz})\end{array}$ & Ref. \\
\hline $\mathrm{V}^{4+}$ & 1.915 & 1.956 & 1.913 & 92.9 & 425.7 & 128.9 & {$[3.5]$} \\
\hline $\mathrm{Nb}^{4+}$ & 1.973 & 1.981 & 1.948 & 5.4 & 24.0 & 6.3 & {$[3.6,3.7]$} \\
\hline $\mathrm{Ta}^{4+}$ & 1.979 & 1.979 & 1.945 & 7.5 & 7.5 & 8.1 & {$[3.6]$} \\
\hline $\mathrm{Mo}^{5+}$ & 1.816 & 1.917 & 1.792 & 71.9 & 197.9 & 89.9 & {$[3.8]$} \\
\hline $\mathrm{W}^{5+}$ & 1.473 & 1.594 & 1.443 & 122.9 & 278.8 & 191.9 & {$[3.9]$} \\
\hline $\mathrm{Ce}^{3+}$ & 2.069 & 4.394 & 3.866 & - & - & - & {$[3.6]$} \\
\hline $\mathrm{Ru}^{3+}$ & 2.822 & 1.845 & 1.015 & 101.9 & - & 78.4 & {$[3.10]^{*}$} \\
\hline $\mathrm{Ru}^{5+}$ & 5.283 & 2.683 & 1.751 & 199.7 & 44.4 & 111.8 & {$[3.10]^{*}$} \\
\hline $\mathrm{Mn}^{4+}$ & 1.988 & 1.985 & 1.985 & - & - & - & {$[3.11]$} \\
\hline $\mathrm{Mn}^{4+}$ & & 1.990 & & - & - & - & {$[3.12]$} \\
\hline
\end{tabular}

* Note: The hyperfine constant is for ${ }^{99} \mathrm{Ru}$. 
usually not found in as-grown $\mathrm{TiO}_{2}$ crystals; they appear as a result of intentional doping during growth or in-diffusion treatments after growth. All these impurities are initially $\mathrm{M}^{4+}, \mathrm{M}^{5+}$, or $\mathrm{M}^{6+}$ ions and they easily replace a $\mathrm{Ti}^{4+}$ ion in the $\mathrm{TiO}_{2}$ lattice. During laser illumination or X-ray-irradiation, these ions trap an electron and convert to $\mathrm{M}^{3+}, \mathrm{M}^{4+}$, or $\mathrm{M}^{5+}$ ions. Thus, the $\mathrm{g}$ matrix of these impurities exhibits the same symmetry as the $\mathrm{TiO}_{2}$ crystal, i.e., the high-symmetry directions in the crystal are the principal axes of the $g$ matrix. More details about these impurity centers can be found in the references.

\subsection{Impurities at Low-Symmetry Sites}

The trivalent impurities $\mathrm{Al}^{3+}$ and $\mathrm{Ga}^{3+}$ substitute for $\mathrm{Ti}^{4+}$ ions in the $\mathrm{TiO}_{2}$ crystal and, like $\mathrm{Fe}^{3+}$ and $\mathrm{Cr}^{3+}$, provide charge compensation for oxygen vacancies. At very low temperature, these trivalent ions provide a potential well that traps holes when electrons are trapped elsewhere. The $\mathrm{Fe}^{3+}$ and $\mathrm{Cr}^{3+}$ change into unobservable $\mathrm{Fe}^{4+}$ and $\mathrm{Cr}^{4+}$ charge states after trapping a hole. In the case of the $\mathrm{Al}^{3+}$ and $\mathrm{Ga}^{3+}$ ions, the hole will be trapped on one of the four nearest-neighbor oxygen ions. The EPR centers, in these latter cases,

Table 3.3. Spin-Hamiltonian parameters of defects associated with trivalent impurities in $\mathrm{TiO}_{2}$ crystals.

\begin{tabular}{|c|c|c|c|c|c|c|c|}
\hline Impurity & $\mathrm{g}_{\mathrm{x}}{ }^{*}$ & $\mathrm{~g}_{\mathrm{y}}{ }^{*}$ & $\mathrm{~g}_{\mathrm{z}}{ }^{*}$ & $\begin{array}{c}\mathrm{A} \\
(\mathrm{MHz})\end{array}$ & $\begin{array}{c}|\mathrm{E}| \\
(\mathrm{GHz})\end{array}$ & $\begin{array}{c}\mathrm{D} \\
(\mathrm{GHz})\end{array}$ & Ref. \\
\hline $\mathrm{Al}^{3+}$ & 2.019 & 2.003 & 2.022 & - & - & - & {$[3.13]$} \\
\hline $\mathrm{Ga}^{3+}$ & 2.030 & 2.023 & 2.007 & - & - & - & {$[3.14]$} \\
\hline $\mathrm{Ni}^{3+}$ & 2.085 & 2.084 & 2.254 & - & - & - & {$[3.15]$} \\
\hline $\mathrm{Ni}^{2+}$ & 2.10 & 2.10 & 2.20 & - & 4.125 & -250 & {$[3.15]$} \\
\hline
\end{tabular}

* The directions of $\mathrm{x} \mathrm{y} \mathrm{z}$ are not high-symmetry directions of the crystal. More details can be found in the corresponding references. 
are thus holelike, i.e., they have g values slightly bigger than 2.0023 , the value for a free electron. The direction pointing from the $\mathrm{Al}^{3+}$ or $\mathrm{Ga}^{3+}$ ion to the oxygen ion with the trapped hole is not one of the three high-symmetry directions in the crystal. In general, the principal axes of the g matrix have arbitrary directions relative to the crystal axes [3.16]. A similar situation applies to $\mathrm{Ni}^{2+}$ and $\mathrm{Ni}^{3+}$ EPR centers in $\mathrm{TiO}_{2}$ [3.15].

\subsection{Native Defects}

Unlike impurities with characteristic hyperfine spectra, the native point defects in $\mathrm{TiO}_{2}$ are very difficult to identify. Possible examples of native defects in this material include vacancies and interstitials. It is generally accepted that titanium vacancies and oxygen interstitials do not easily form in the $\mathrm{TiO}_{2}$ lattice. It is, however, well known that reducing a $\mathrm{TiO}_{2}$ crystal in an oxygen-deficient environment will change the insulator into an n-type semiconductor. Explanations for this latter phenomenon are still controversial. It is generally accepted that oxygen leaves the crystal in neutral atom (molecule) form when the crystal is reduced. The simplest mechanism that can occur during a reducing treatment is to have oxygen vacancies form as oxygen atoms leave the crystal. However, many researchers believe that interstitial titanium ions are formed during reducing [3.17].

In either case (oxygen vacancies or titanium interstitials), $\mathrm{Ti}^{3+}$ ions are expected to be observed in subsequent EPR experiments. Thus, it is not trivial to obtain evidence that will distinguish between the two mechanisms. The situation becomes even more complicated because impurities in the $\mathrm{TiO}_{2}$ lattice may trap electrons and form adjacent $\mathrm{Ti}^{3+}$ ions. EPR signals from at least six different defects involving $\mathrm{Ti}^{3+}$ ions have been observed in $\mathrm{TiO}_{2}$. Earlier investigators have been unable to unambiguously assign EPR signals to oxygen vacancies or $\mathrm{Ti}^{3+}$ interstitials.

In 1961, Chester [3.18] reported a $\mathrm{Ti}^{3+}$ EPR signal, which he referred to as the ' $\mathrm{A}$ ' center. This center was observed in a reduced sample (i.e., one heated in an oxygendeficient atmosphere). This signal was only observed at very low temperature (below 10 
$\mathrm{K})$. The $\mathrm{g}$ values for this center are $\mathrm{g}_{\mathrm{x}}=1.974, \mathrm{~g}_{\mathrm{y}}=1.977$, and $\mathrm{g}_{\mathrm{z}}=1.941$. The principal direction of $g_{z}$ is along the [001] direction in the crystal and the principal direction of $g_{x}$ lies in the plane perpendicular to the [001] axis and making an angle of $26^{\circ}$ with the [100] direction in the crystal. According to Chester [3.18], $\mathrm{a} \mathrm{Ti}^{3+}$ interstitial was one of the possible models based on his belief that there were four crystallographically equivalent sites in the EPR spectrum. This interstitial model has been subsequently cited by many researchers. Chester, however, also proposed two additional models for this $\mathrm{Ti}^{3+}$ defect, and stated that a specific model could not be established based on his EPR data alone. Another important EPR investigation of defects in $\mathrm{TiO}_{2}$ was published by Kerssen and Volger [3.19]. Electronlike centers, one referred as B and another referred to as B1 and $\mathrm{B} 2$ were reported. $\mathrm{B} 1$ and $\mathrm{B} 2$ are the two EPR signals that comprise a spin $\mathrm{S}=1$ system. In their initial spin-Hamiltonian analysis, $\mathrm{B} 1$ and $\mathrm{B} 2$ were treated as two $\mathrm{S}=1 / 2$ centers. Kerssen and Volger assigned their EPR spectra to interstitial $\mathrm{Ti}^{3+}$ ions with adjacent $\mathrm{Al}^{3+}$ ions, but these models are questionable because no hyperfine due to ${ }^{27} \mathrm{Al}$ nuclei were observed. Now it is known that a hole will be trapped near $\mathrm{Al}^{3+}$ and form a hole center when $\mathrm{TiO}_{2}$ contains aluminum impurities [3.13]. 


\section{Chapter 4}

\section{EPR Study of Oxygen Vacancies and Silicon Impurities}

\subsection{Introduction}

In the previous chapter, I described many of the early EPR investigations of point defects in $\mathrm{TiO}_{2}$ crystals. Most of these EPR studies focused on impurities, while a few were concerned with native defects in reduced crystals. These latter studies raised significant questions as to the nature of the paramagnetic defects generated by reducing treatments (i.e., are they interstitial titanium ions or are they oxygen vacancies) [4.1-4.4]. In the present chapter, I provide answers to these questions by describing EPR results from colorless (fully oxidized) $\mathrm{TiO}_{2}$ crystals that contain small concentrations of doubly ionized oxygen vacancies. These doubly ionized vacancies provide charge compensation for the trace amounts of trivalent transition-metal-ion impurities that are inadvertently present in my $\mathrm{TiO}_{2}$ crystals. When describing the different charge states of the oxygen

vacancy, I use doubly ionized $\left(\mathrm{V}_{\mathrm{O}}^{++}\right)$, singly ionized $\left(\mathrm{V}_{\mathrm{O}}^{+}\right)$, and neutral $\left(\mathrm{V}_{\mathrm{O}}^{0}\right)$ to refer to a vacancy that is unoccupied, singly occupied, and doubly occupied, respectively, with trapped electrons.

In this chapter, the singly ionized $(S=1 / 2)$ and the neutral $(S=1)$ charge states of oxygen vacancies in $\mathrm{TiO}_{2}$ crystals are identified using EPR. These centers appear when crystals containing doubly ionized oxygen vacancies are exposed at low temperature to $442 \mathrm{~nm}$ laser light. Electrons trapped at the oxygen vacancies are localized on two of the three neighboring titanium ions. Also, in this chapter, a defect consisting of an electron trapped on a titanium ion adjacent to a substitutional silicon impurity is identified.

\subsection{Experimental Details}

The three $\mathrm{TiO}_{2}$ crystals used in the studies reported in this chapter have the rutile structure. Two were grown by CrysTec (Germany) and had dimensions of $2.5 \times 3.0$ x 2.0 
$\mathrm{mm}^{3}$ and $3.8 \times 5.0 \times 0.5 \mathrm{~mm}^{3}$. One was grown by Namiki (Japan) and had dimensions of $2.5 \times 3.5 \times 1.2 \mathrm{~mm}^{3}$. In their as-received state, these samples were colorless at room temperature, having been annealed in air after growth. Although the results are not presented here, similar photoinduced EPR experiments were performed on numerous additional $\mathrm{TiO}_{2}$ samples. All of these samples exhibited EPR signals from oxygen vacancies. The concentrations of the oxygen vacancies, however, varied from sample to sample, and the concentrations of the trivalent transition-metal ions $\left(\mathrm{Fe}^{3+}\right.$ and $\left.\mathrm{Cr}^{3+}\right)$ also varied from sample to sample. I found that the concentrations of trivalent ions and the concentrations of oxygen vacancies were correlated, i.e., samples with more trivalent transition-metal ions had more oxygen vacancies.

The Bruker EMX EPR spectrometer operated at a microwave frequency near $9.427 \mathrm{GHz}$ (unless otherwise specified). During the EPR measurements, the temperature of the sample was maintained between 10 and $40 \mathrm{~K}$. Approximately $15 \mathrm{~mW}$ of $442 \mathrm{~nm}$ light from a He-Cd laser was incident on the sample during the low-temperature illuminations. Two other wavelengths were also used in this study; $325 \mathrm{~nm}$ light from the same $\mathrm{He}-\mathrm{Cd}$ laser and $633 \mathrm{~nm}$ light from a He-Ne laser. All three wavelengths produced similar EPR signals in the $\mathrm{TiO}_{2}$ crystals.

\section{3. $\mathrm{Fe}^{3+}$ and $\mathrm{Cr}^{3+}$ Impurity Ions}

At room temperature, all of the as-received $\mathrm{TiO}_{2}$ samples showed several narrow and intense EPR signals from $\mathrm{Fe}^{3+}$ and $\mathrm{Cr}^{3+}$ ions substituting for $\mathrm{Ti}^{4+}$ ions [4.5-4.8]. With the magnetic field parallel to the [001] axis, a signal due to $\mathrm{Cr}^{3+}$ ions was observed near $1377 \mathrm{G}$ and two signals due to $\mathrm{Fe}^{3+}$ ions were observed near $843 \mathrm{G}$ and $9050 \mathrm{G}$. EPR signals from $\mathrm{Fe}^{3+}$ and $\mathrm{Cr}^{3+}$ sharpen (and thus become more intense) at low temperature (e.g., $30 \mathrm{~K}$ ) because the spin-lattice relaxation time becomes longer. This allows the hyperfine structure that accompanies the signals to be easily observed. A spectrum that includes the $\mathrm{Fe}^{3+}(843 \mathrm{G})$ and $\mathrm{Cr}^{3+}(1377 \mathrm{G})$ signals was shown earlier in Fig. 3.1 in 
Chapter 3. Figures 4.1 and 4.2 show the hyperfine structure associated with these two signals in detail, i.e., the structure caused by the ${ }^{57} \mathrm{Fe}$ and ${ }^{53} \mathrm{Cr}$ nuclei. The two spectra in Figs. 4.1 and 4.2 were taken from the CrysTec sample with dimensions of $3.0 \times 3.0 \times 2.0$ $\mathrm{mm}^{3}$. The spectrum in Fig. 4.1(a) shows four equally spaced EPR lines around the large center EPR line. The separations between these four lines is approximately $18 \mathrm{G}$. Experimentally, I found the ratio of the intensity of one of these four lines to the intensity of the larger center line to be 1 to 37.9 . The ${ }^{53} \mathrm{Cr}$ nuclei have a nuclear spin of $\mathrm{I}=3 / 2$ and are $9.50 \%$ abundant. This predicts that an EPR spectrum resulting from a chromium ion will have a four-line hyperfine pattern surrounding the more intense center EPR line, and that the ratio of a hyperfine line to the middle line will be 1 to 38.1 . Thus, a comparison of the ratio of the experimental intensities with the predicted ratio conclusively proves that the EPR spectrum in Fig. 4.1 is due to $\mathrm{Cr}^{3+}$ ions. The spectrum in Fig. 4.1(b) shows superhyperfine structure due to the neighboring ${ }^{47} \mathrm{Ti}$ and ${ }^{49} \mathrm{Ti}$ nuclei. The titanium hyperfine pattern contains eight lines (see the stick diagram in Fig. 4.1(b)), where the two outer lines are half the intensity of the six inner lines. In Fig. 4.1(a), two hyperfine lines in the middle of the spectrum are hidden by the large $\mathrm{I}=0$ center line. The weaker hyperfine structure shown in Fig. 4.1(b) indicates that the $\mathrm{Cr}^{3+}$ ion interacts equally with the two $\mathrm{Ti}^{4+}$ ions along the [001] direction that are located above and below the $\mathrm{Cr}^{3+}$ ion. This superhyperfine spectrum supports the model of a $\mathrm{Cr}^{3+}$ ion substituting for a $\mathrm{Ti}^{4+}$ ion. Figure 4.2 shows the hyperfine structure associated with the $\mathrm{Fe}^{3+}$ EPR signal. The two small hyperfine lines (separated by $12 \mathrm{G}$ ) verify that this EPR signal is due to a $\mathrm{Fe}^{3+}$ ion. The eight closely spaced and only partially resolved superhyperfine signals are due to $\mathrm{Ti}^{4+}$ ions. Together, they indicate that the $\mathrm{Fe}^{3+}$ ion substitutes for a $\mathrm{Ti}^{4+}$ ion.

As discussed in Chapter 3, it is generally accepted [4.9] that isolated substitutional $\mathrm{Fe}^{3+}$ and $\mathrm{Cr}^{3+}$ impurities in $\mathrm{TiO}_{2}$ are charge compensated by remotely located oxygen vacancies (i.e., one doubly ionized oxygen vacancy compensates two trivalent transition- 

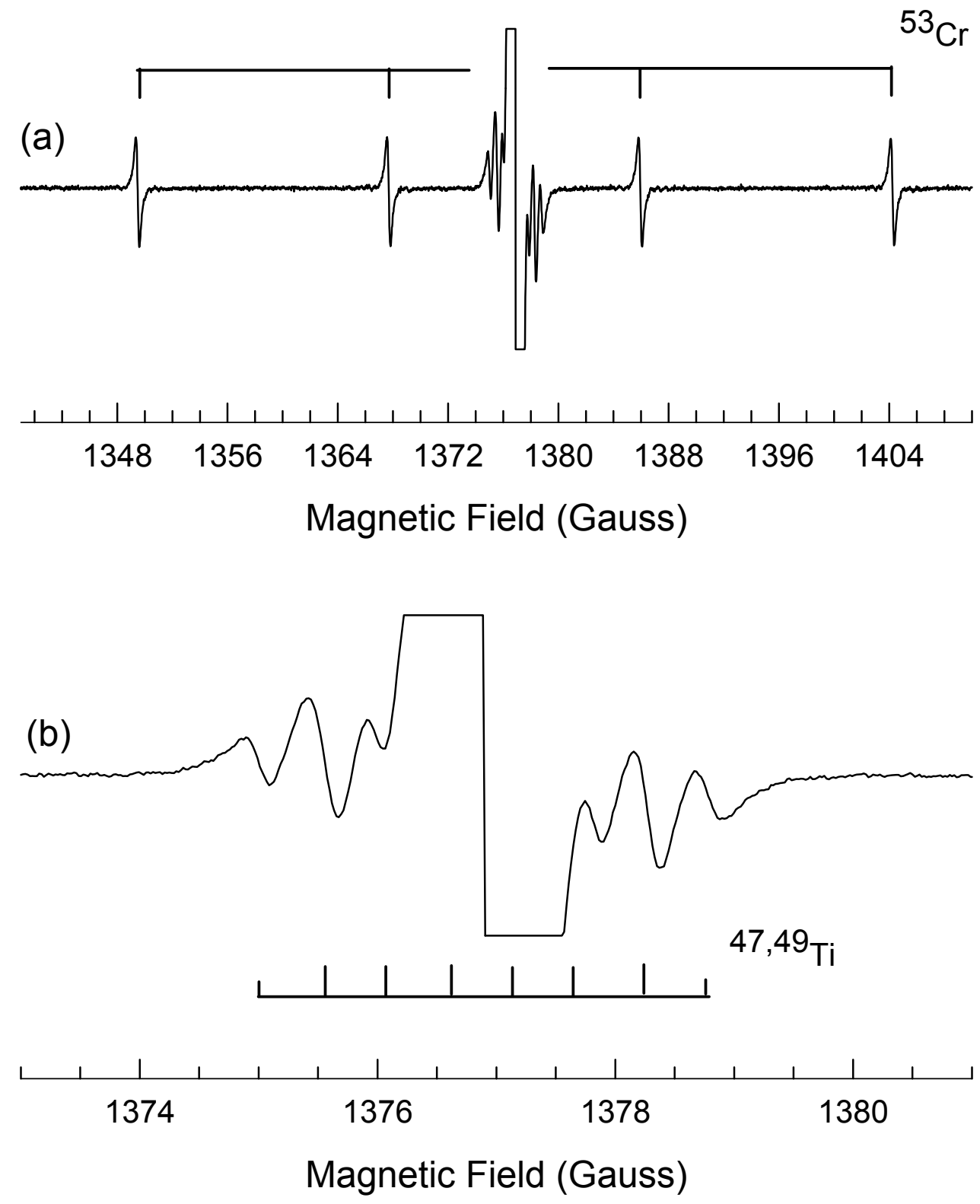

Figure 4.1. EPR spectra showing hyperfine associated with $\mathrm{Cr}^{3+}$ ions in $\mathrm{TiO}_{2}$ crystals. These data were taken at $30 \mathrm{~K}$, the magnetic field was parallel to the [001] axis, and the microwave frequency was near $9.59 \mathrm{GHz}$. Trace (a) shows the hyperfine due to the ${ }^{53} \mathrm{Cr}$ nucleus $(\mathrm{I}=3 / 2)$. Trace $(\mathrm{b})$ shows the hyperfine due to the nearest neighbor ${ }^{47} \mathrm{Ti}(\mathrm{I}=5 / 2)$ and ${ }^{49} \mathrm{Ti}(\mathrm{I}=7 / 2)$ nuclei. 
metal ions replacing $\mathrm{Ti}^{4+}$ ions). It is also possible that some of the substitutional $\mathrm{Fe}^{3+}$ and $\mathrm{Cr}^{3+}$ ions are charge compensated by $\mathrm{OH}^{-}$molecular ions. A study was performed to see if the concentration of oxygen vacancies in a sample correlated with the concentration of trivalent $\mathrm{Fe}^{3+}$ and $\mathrm{Cr}^{3+}$ ions in the same sample. A Namiki sample and a CrysTec sample (the one with $0.5 \mathrm{~mm}$ thickness) were used in this comparison. Our EPR results show that the Namiki sample has a larger concentration of $\mathrm{Fe}^{3+}$ and $\mathrm{Cr}^{3+}$ than the CrysTec sample and the Namiki sample also has a larger concentration of oxygen vacancies than the CrysTec sample. The combined concentration of these $\mathrm{Fe}^{3+}$ and $\mathrm{Cr}^{3+}$ impurities,

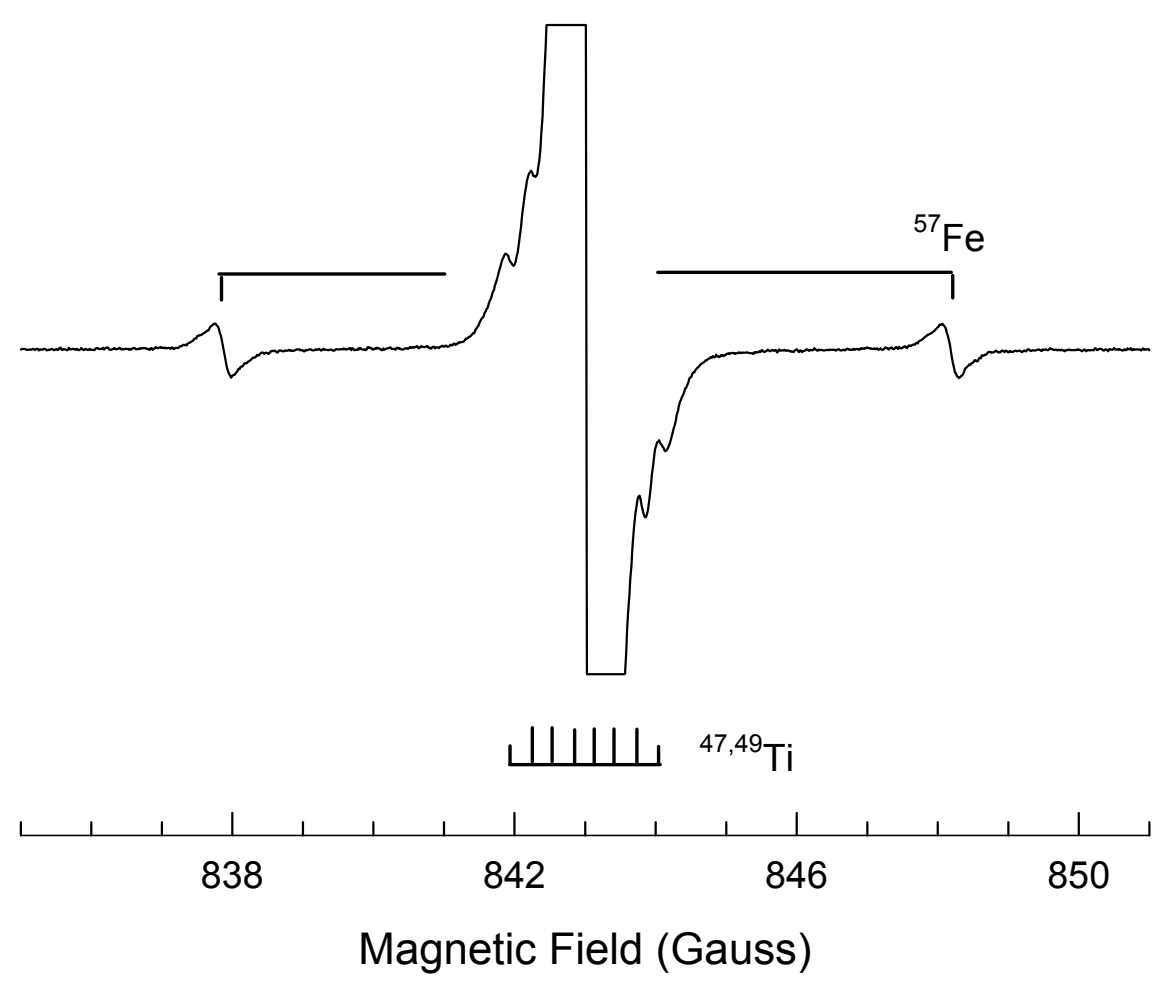

Figure 4.2. EPR hyperfine from $\mathrm{Fe}^{3+}$ ions in $\mathrm{TiO}_{2}$ crystals. These data were taken at $30 \mathrm{~K}$, the magnetic field was parallel to the [001] axis, and the microwave frequency was near $9.59 \mathrm{GHz}$. The two hyperfine lines separated by $12 \mathrm{G}$ are due to ${ }^{57} \mathrm{Fe}(\mathrm{I}=1 / 2)$ nuclei, and the hyperfine lines near the center line are due to ${ }^{47} \mathrm{Ti}(\mathrm{I}=5 / 2)$ and ${ }^{49} \mathrm{Ti}(\mathrm{I}=7 / 2)$ nuclei. 
estimated from the intensity of their EPR signals, is approximately one part per million in the Namiki sample and one-tenth of this value in the CrysTec sample. These estimates of the absolute concentrations of $\mathrm{Fe}^{3+}$ and $\mathrm{Cr}^{3+}$ ions are based on a comparison with a weak pitch sample supplied by Bruker (error limits are $\pm 30 \%$ ).

\subsection{Paramagnetic Oxygen Vacancies and $\mathrm{Ti}^{3+}-\mathrm{Si}^{4+}$ Centers}

Our $\mathrm{TiO}_{2}$ samples did not show EPR signals at low temperature $(10$ to $40 \mathrm{~K})$ in the magnetic field region from 3300 to $4000 \mathrm{G}$ when they were initially cooled in the dark. Subsequent illumination at low temperature, however, immediately produced easily observed trapped-electron centers. The EPR data in Fig. 4.3 were taken from the Namiki sample with the magnetic field parallel to the [001] axis. Exposure of the sample to $442 \mathrm{~nm}$ laser light at $26 \mathrm{~K}$ produces three sharp EPR lines (two lines are shown in Fig. 4.3(a) while a third EPR line near $3958 \mathrm{G}$ is not shown). The lowest-field line near 3419 $\mathrm{G}$ and the highest-field line near $3958 \mathrm{G}$ (not shown) belong to an $\mathrm{S}=1$ center with $\mathrm{g}_{[001]}$ $=1.826$. $\left(\mathrm{g}_{[001]}\right.$ denotes the $\mathrm{g}$ value when the magnetic field is parallel to the $[001]$ axis.) These two lines represent the $M_{S}=-1$ to 0 and the $M_{S}=0$ to +1 transitions. A detailed angular dependence study of this spectrum, described later in this chapter, provides complete sets of spin-Hamiltonian parameters. This $\mathrm{S}=1$ spectrum is assigned to the neutral charge state of the oxygen vacancy $\left(V_{O}^{0}\right)$. It was produced in our study when a doubly ionized oxygen vacancy $\left(\mathrm{V}_{\mathrm{O}}^{++}\right)$trapped two photoinduced electrons. These two electrons are localized on the two neighboring titanium ions aligned along the [001] axis. A simple model would have the two trapped electrons separately form neighboring $\mathrm{Ti}^{3+}$ ions that couple to form the $S=1$ spin system. In support of our assignment, we found that a much more intense $S=1$ signal from the neutral oxygen vacancy was present at 26 $\mathrm{K}$ (without light) in a slightly reduced $\mathrm{TiO}_{2}$ crystal (i.e., a crystal held for 30 min at 600 ${ }^{\circ} \mathrm{C}$ in flowing nitrogen gas). This reduction treatment produced uncompensated neutral 

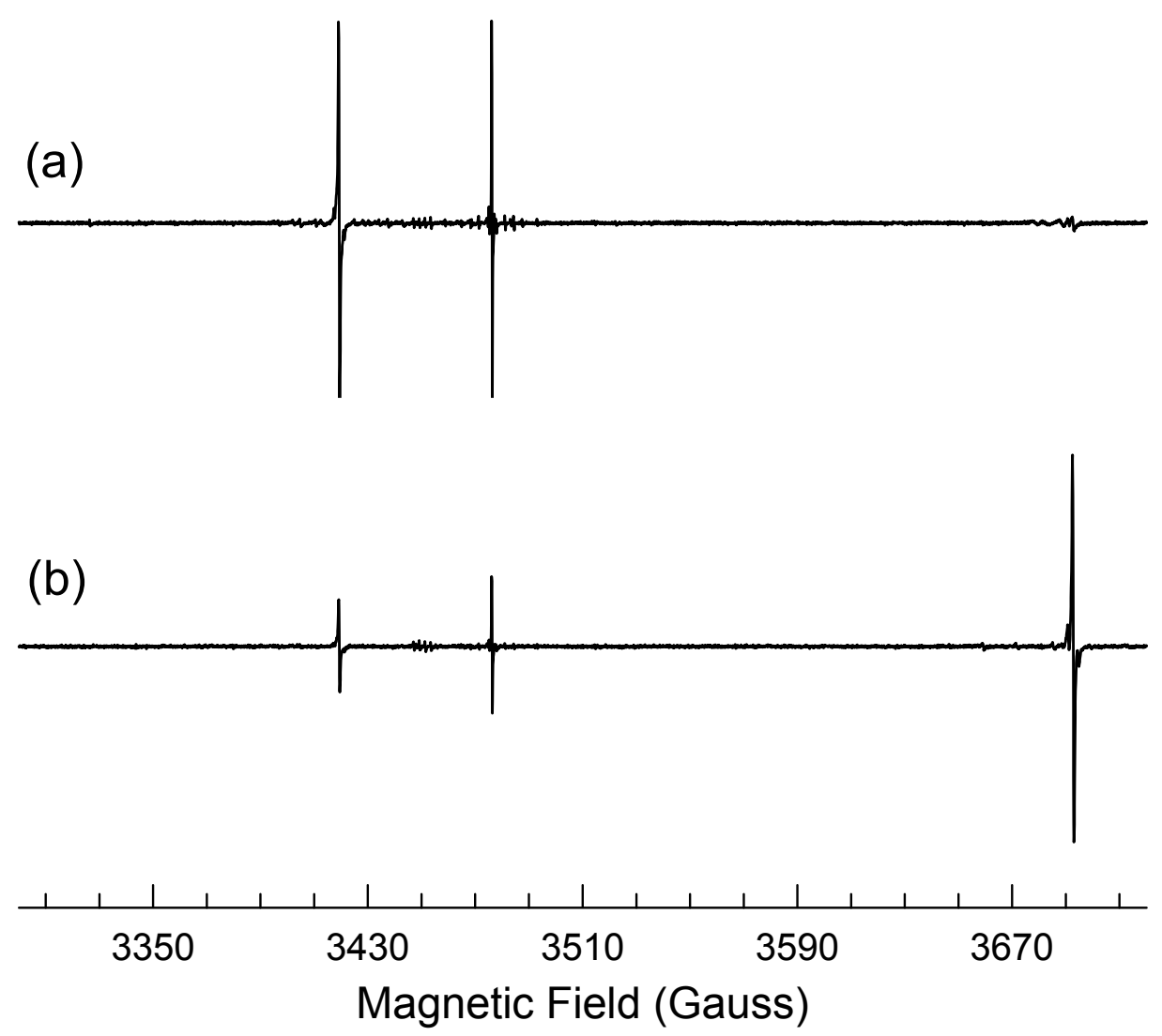

Figure 4.3. EPR spectra taken at $26 \mathrm{~K}$ from a $\mathrm{TiO}_{2}$ crystal. The magnetic field was along the [001] direction. Trace (a) was taken with illumination of $442 \mathrm{~nm}$ laser light. Trace (b) was taken two minutes after the laser light was removed.

oxygen vacancies as oxygen atoms are removed, and laser light was not needed to photoinduce the $\mathrm{S}=1$ spectrum.

We identify the EPR signal near $3476 \mathrm{G}$ in Fig. 4.3(a) as a $\mathrm{Ti}^{3+}-\mathrm{Si}^{4+}$ center. This is an $\mathrm{S}=1 / 2$ defect with $\mathrm{g}_{[001]}=1.938$, and is formed when a photoinduced electron is trapped on a regular $\mathrm{Ti}^{4+}$ ion that has a substitutional $\mathrm{Si}^{4+}$ ion at a nearest cation site along the [001] axis. Figure 4.4 shows the hyperfine structure accompanying this $\mathrm{Ti}^{3+}-\mathrm{Si}^{4+}$ center. In addition to the eight lines caused by the ${ }^{47} \mathrm{Ti}$ and ${ }^{49} \mathrm{Ti}$ isotopes, there is an extra pair of hyperfine lines separated by approximately $2.0 \mathrm{G}$ and symmetrically located about the center line. Within this pair, the left line is slightly more intense than the right line 
because it is overlapped by one of four small closely spaced lines. [Note: Several weak four-line EPR spectra possibly associated with interstitial $\mathrm{Li}^{+}$or $\mathrm{Na}^{+}$ions were observed in the Namiki sample. Their origin will be addressed in Chapter 8.] The extra pair of hyperfine lines in Fig. 4.4 requires that the responsible nucleus has $\mathrm{I}=1 / 2$, and the small hyperfine constant of $2.0 \mathrm{G}$ suggests that this nucleus is located at a neighboring site. The intensities of these two hyperfine lines, when compared to the intensities of the ${ }^{47} \mathrm{Ti}$ and ${ }^{49} \mathrm{Ti}$ hyperfine lines, indicate that the isotopic abundance of the $\mathrm{I}=1 / 2$ nucleus is slightly less than $5 \%$. The only possible candidate is ${ }^{29} \mathrm{Si}$ with an abundance of $4.67 \%$. Elemental analyses of impurities in $\mathrm{TiO}_{2}$ crystals often reveal the presence of silicon [4.3, 4.10].

Figure 4.3(b) shows the EPR spectrum taken at $26 \mathrm{~K}$ shortly after the laser light is removed from the sample. An additional line, representing an $\mathrm{S}=1 / 2$ defect with $\mathrm{g}_{[001]}=$ 1.824, appears near $3693 \mathrm{G}$ and quickly becomes the dominant signal. At the same time, the intensities of the $\mathrm{V}_{\mathrm{O}}^{0}$ and $\mathrm{Ti}^{3+}-\mathrm{Si}^{4+}$ signals decrease significantly. When the laser light is restored while still keeping the sample at $26 \mathrm{~K}$, the line at $3693 \mathrm{G}$ disappears almost instantly and the $\mathrm{V}_{\mathrm{O}}^{0}$ and $\mathrm{Ti}^{3+}-\mathrm{Si}^{4+}$ signals return to their initial intensities. We assign this signal at $3693 \mathrm{G}$ to a $\mathrm{V}_{\mathrm{O}}^{+}$center. Specifically, we suggest that this defect is a singly ionized oxygen vacancy with the trapped electron equally shared by the same two titanium ions that form the $\mathrm{S}=1 \mathrm{~V}_{\mathrm{O}}^{0}$ center (i.e., the two titanium neighbors aligned along the $[001]$ axis). The $\mathrm{V}_{\mathrm{O}}^{0}$ center and the $\mathrm{V}_{\mathrm{O}}^{+}$center have similar $\mathrm{g}$ matrices $\left(\mathrm{g}_{[001]}=\right.$ 1.826 and $\mathrm{g}_{[001]}=1.824$, respectively), which supports our models based on oxygen vacancies. Conversion of a $V_{O}^{0}$ center to a $V_{O}^{+}$center occurs when the shallow $V_{O}^{0}$ center thermally releases one of its two trapped electrons at temperatures near or above $26 \mathrm{~K}$. 


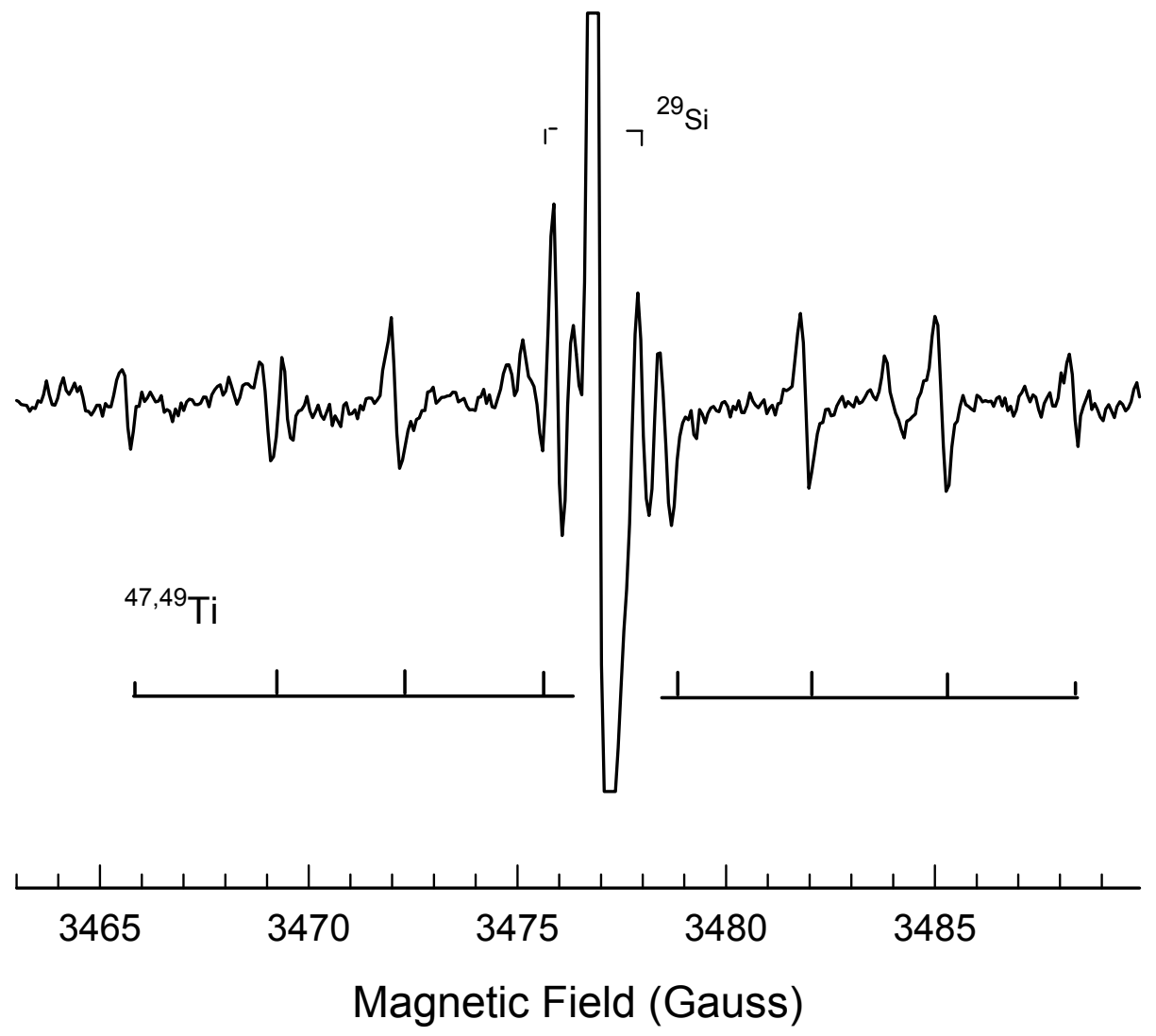

Figure 4.4. EPR spectrum of the $\mathrm{Ti}^{3+}-\mathrm{Si}^{4+}$ center taken at $26 \mathrm{~K}$ with the magnetic field parallel to the [001] direction. The upper and lower stick diagrams show the ${ }^{29} \mathrm{Si}$ and the ${ }^{47} \mathrm{Ti}$ and ${ }^{49} \mathrm{Ti}$ hyperfine lines, respectively. Additional weak lines in the spectrum are unidentified.

\subsection{Spin-Hamiltonian Analysis}

Figure 4.5 describes the EPR angular dependence data from the $\mathrm{V}_{\mathrm{O}}^{0}$ center. The horizontal axis represents the direction of the magnetic field, and the vertical axis gives the values of magnetic field where EPR signals occur. These data were taken while rotating separately in the three high-symmetry planes in the crystal (from [100] to [001], from [001] to [110], and from [110] to [1-10]). For each plane, care was taken to mount the crystal with the appropriate crystal direction perpendicular to the plane of rotation. 


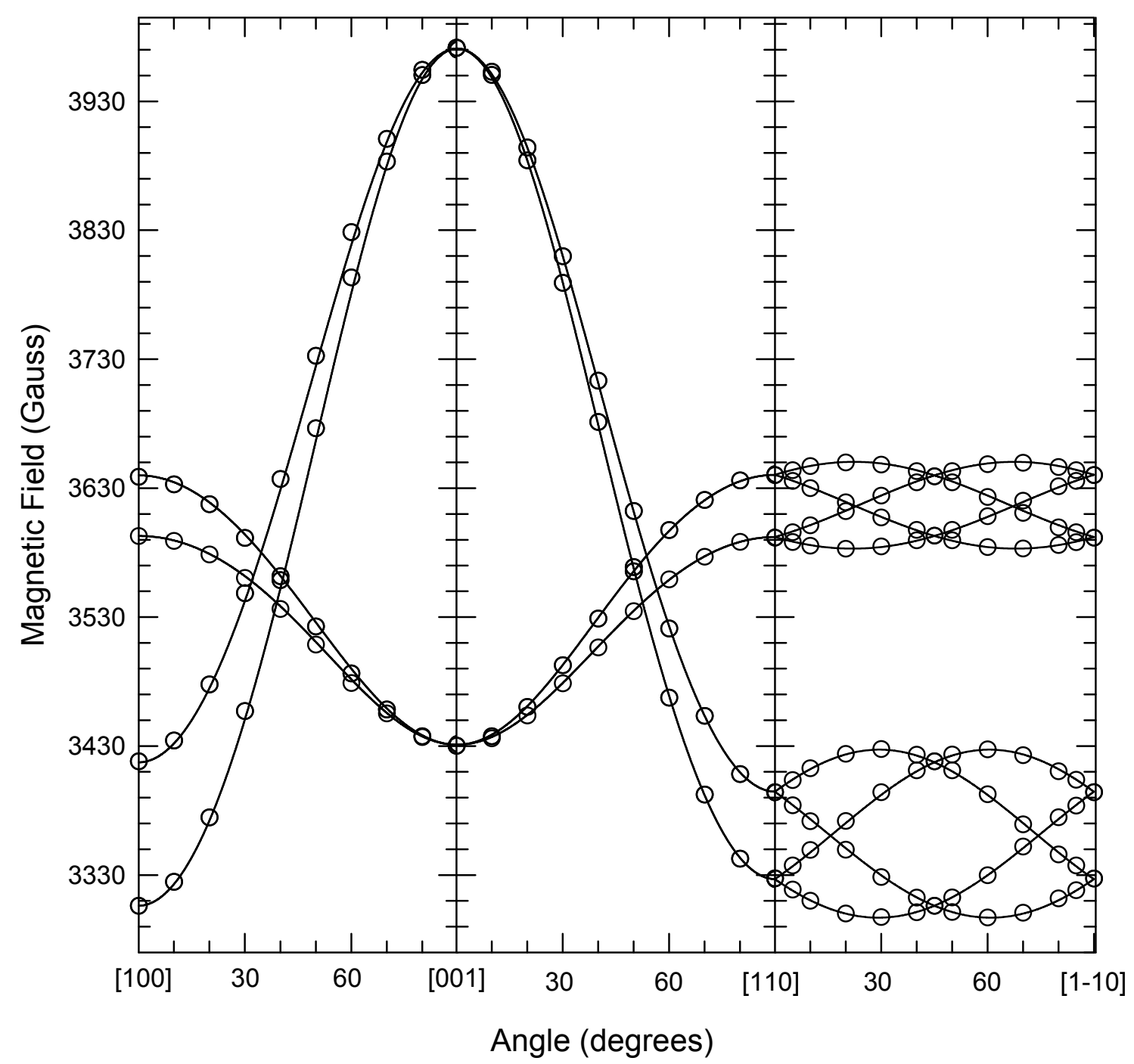

Figure 4.5. Angular dependence of the $g$ and $\mathrm{D}$ matrices for the neutral oxygen vacancy. These EPR data were acquired in the three high-symmetry planes of the crystal. The discrete open circles are experimental data, while the solid curves are computer generated using the best fit parameters.

For example, when rotating from [100] to [001], the sample was mounted in the microwave cavity with the [010] direction vertical, thus allowing the direction of the magnetic field to be varied from [100] to [001]. The open circles in Fig. 4.5 represent experimental data taken in steps of $10^{\circ}$ in each of the three planes (the complete set of 
experimental data is presented in Appendix B). Uncertainty in the angular positions is less than 1 degree. The solid curves in Fig. 4.5 are computer-generated using the final set of best parameters, which were produced by a least-squares fitting program. The follow-

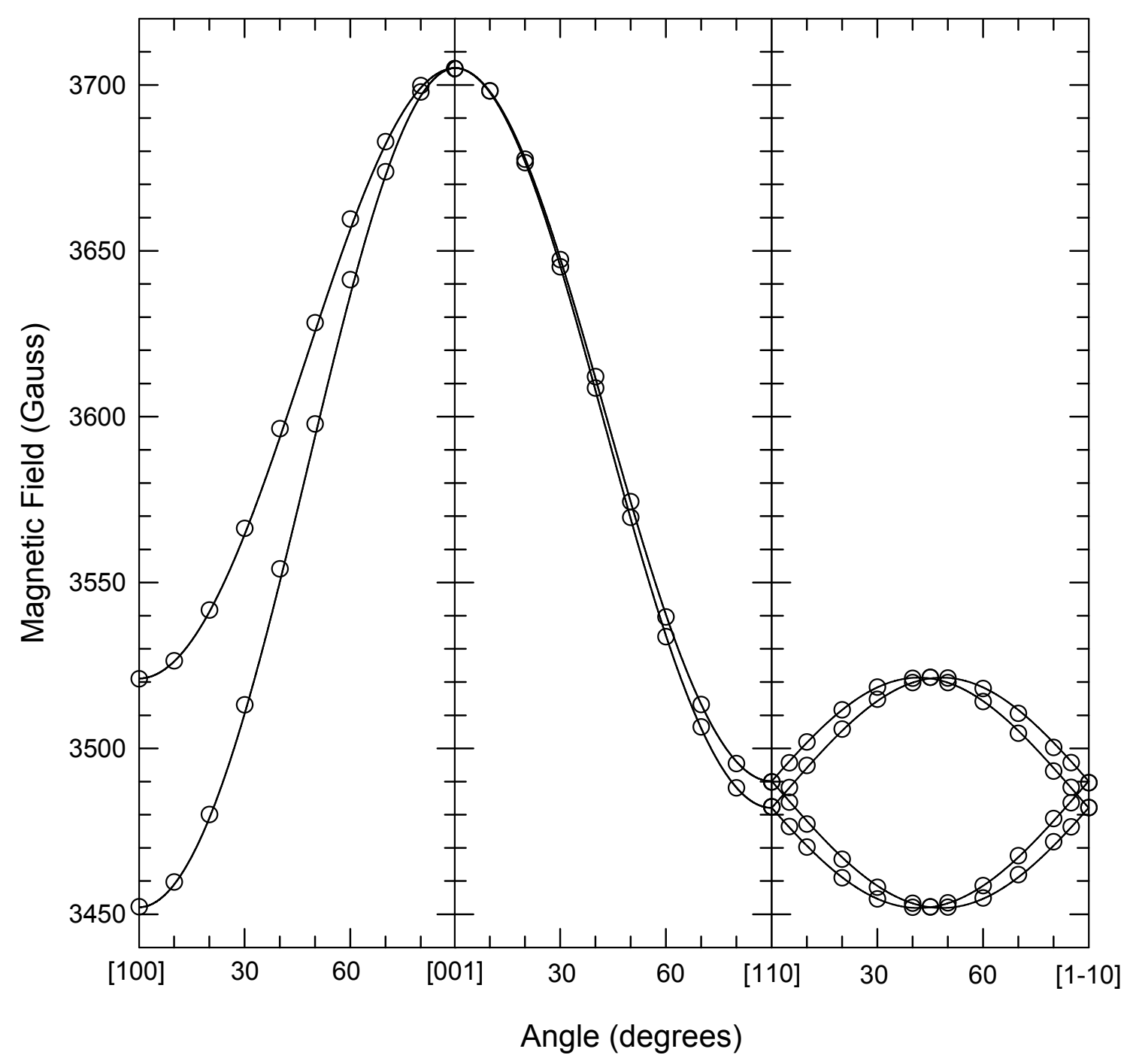

Figure 4.6. Angular dependence of the g matrix for the singly ionized oxygen vacancy center. These EPR data were acquired in the three high-symmetry planes of the crystal. The discrete open circles are experimental data, while the solid curves are computer generated using the best fit parameters. 
ing spin Hamiltonian was used to analyze the angular dependence data of the $\mathrm{V}_{\mathrm{O}}^{0}$ center.

$$
\mathbf{H}=\beta \mathbf{S} \cdot \mathbf{g} \cdot \mathbf{B}+\mathbf{S} \cdot \mathbf{D} \cdot \mathbf{S}
$$

The electron Zeeman term is $\beta \mathbf{S} \cdot \mathbf{g} \cdot \mathbf{B}$ and the fine structure (zero-field-splitting) term is $\mathbf{S} \cdot \mathbf{D} \cdot \mathbf{S}$ where $\mathbf{D}$ is a traceless matrix (the sum of the diagonal elements is zero).

Figure 4.6 describes the EPR angular dependence data from the $\mathrm{V}_{\mathrm{O}}^{+}$center (the complete set of experimental data is presented in Appendix B). The following spin Hamiltonian was used to fit this data.

$$
\mathbf{H}=\beta \mathbf{S} \cdot \mathbf{g} \cdot \mathbf{B}
$$

The angular dependence data for the $\mathrm{V}_{\mathrm{O}}^{0}$ and $\mathrm{V}_{\mathrm{O}}^{+}$centers, shown in Figs. 4.5 and 4.6, have very similar behaviors. Each line in the angular dependence of the $\mathrm{S}=1 / 2 \mathrm{~V}_{\mathrm{O}}^{+}$ center becomes two lines in the angular dependence of the $\mathrm{S}=1 \mathrm{~V}_{\mathrm{O}}^{0}$ center. The lines in the $\mathrm{V}_{\mathrm{O}}^{+}$center are centered on the corresponding lines in the $\mathrm{V}_{\mathrm{O}}^{0}$ center. These nearly

Table 4.1. Spin-Hamiltonian parameters of the $\mathrm{V}_{\mathrm{O}}^{0}$ center and the $\mathrm{V}_{\mathrm{O}}^{+}$center. These parameters are generated by fitting the EPR angular dependence data using a least-squares MATLAB program. The angle $\phi$ is relative to the [100] direction.

\begin{tabular}{|c|c|c|c|c|}
\hline \multirow{2}{*}{ Center } & \multicolumn{2}{|c|}{ Principal Values } & \multicolumn{2}{c|}{ Principal Axes } \\
\cline { 3 - 5 } & \multicolumn{2}{|c|}{$\theta(\mathrm{deg})$} & $\phi(\mathrm{deg})$ \\
\hline \multirow{3}{*}{$\mathrm{V}_{\mathrm{O}}^{0}$} & $\mathrm{~g}_{1}$ & 1.9581 & 90.0 & 3.8 \\
\cline { 2 - 5 } & $\mathrm{g}_{2}$ & 1.9136 & 90.0 & 93.8 \\
\cline { 2 - 5 } & $\mathrm{g}_{3}$ & 1.8260 & 0.0 & 0.0 \\
\cline { 2 - 5 } & $\mathrm{D}_{1}$ & 291.52 & 90 & 60.5 \\
\cline { 2 - 5 } & $\mathrm{D}_{2}$ & 168.51 & 90 & 150.5 \\
\cline { 2 - 5 } & $\mathrm{D}_{3}$ & -460.03 & 0.0 & 0.0 \\
\hline \multirow{3}{*}{$\mathrm{V}_{\mathrm{O}}^{+}$} & $\mathrm{g}_{1}$ & 1.9578 & 90.0 & 3.3 \\
\cline { 2 - 5 } & $\mathrm{g}_{2}$ & 1.9192 & 90.0 & 93.3 \\
\cline { 2 - 5 } & $\mathrm{g}_{3}$ & 1.8240 & 0.0 & 0.0 \\
\hline
\end{tabular}




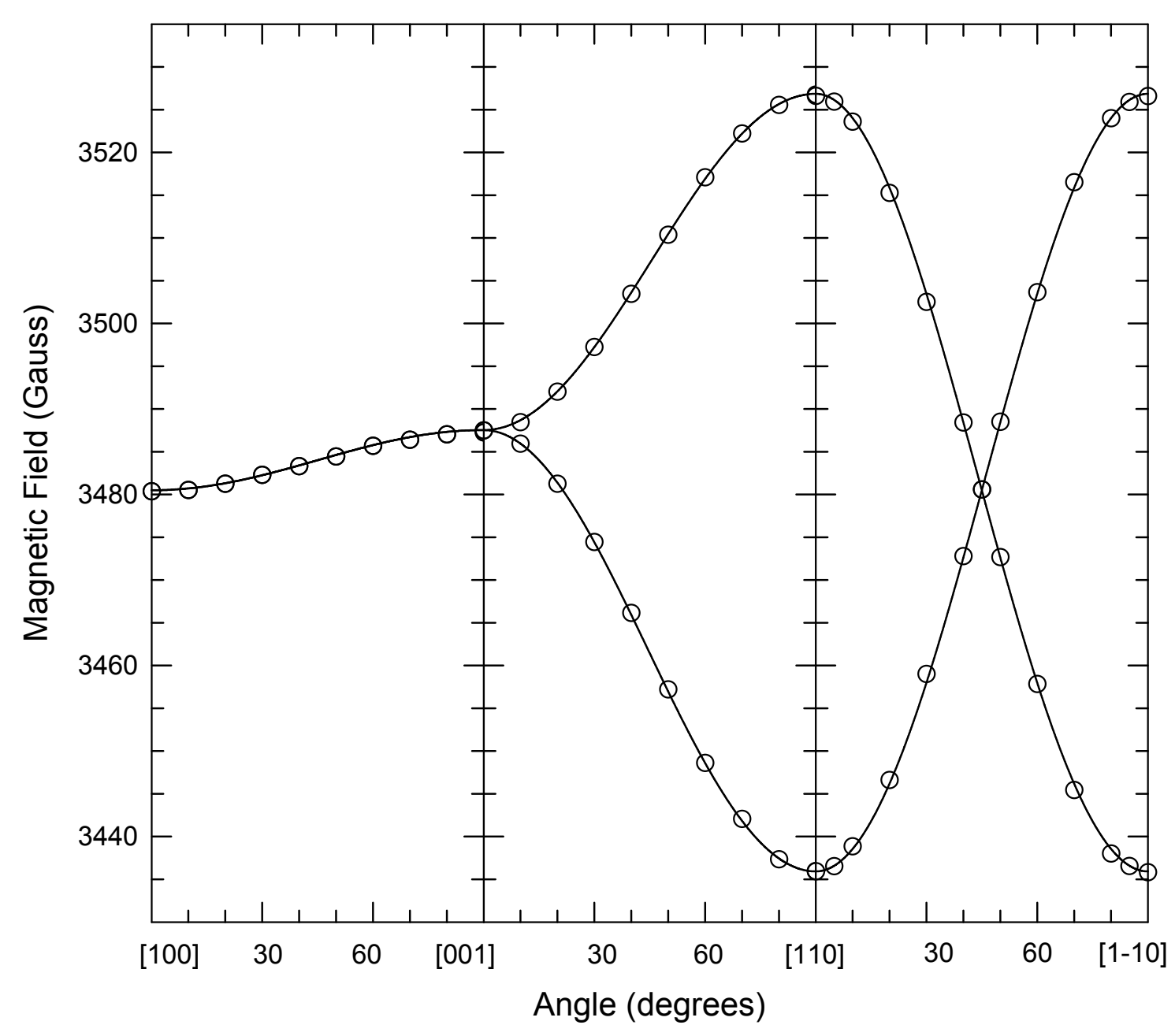

Figure 4.7. EPR angular dependence data from the $\mathrm{Ti}^{3+}-\mathrm{Si}^{4+}$ center. These EPR data were acquired in the three high-symmetry planes of the crystal. The discrete open circles are experimental data, while the solid curves are computer generated using the best fit parameters.

identical angular dependences suggest that the two centers have similar models. The spin-Hamiltonian parameters of the $\mathrm{V}_{\mathrm{O}}^{0}$ and $\mathrm{V}_{\mathrm{O}}^{+}$centers are listed in Table 4.1. The $\mathrm{g}$ matrices of these two centers have almost the same principal values and similar principalaxis directions.

Normally, six parameters including three principal values and three principal directions (represented by three Euler angles) are needed to describe an $\mathrm{S}=1 / 2$ system, 
and a total of eleven parameters are needed for an $\mathrm{S}=1$ system. An $\mathrm{S}=1$ system contains a g matrix and a $\mathrm{D}$ matrix. The angular dependence data from the $\mathrm{V}_{\mathrm{O}}^{0}$ and $\mathrm{V}_{\mathrm{O}}^{+}$ centers show that the EPR signals are at turning points when the magnetic field is along the [001] direction in the crystal. Thus, only four parameters are needed to describe the $\mathrm{V}_{\mathrm{O}}^{+}$center $(\mathrm{S}=1 / 2)$, including three principal values and one angle $\phi$ relative to the [100] direction in the plane perpendicular to [001] axis (the other two Euler angles are zero). Similarly, seven parameters are needed to describe the $\mathrm{V}_{\mathrm{O}}^{0}(\mathrm{~S}=1)$ center. For both the $\mathrm{V}_{\mathrm{O}}^{0}$ and the $\mathrm{V}_{\mathrm{O}}^{+}$centers, four crystallographically equivalent sites are needed to analyze their angular dependences. The Euler angle $\phi$ for one of these sites is related to the Euler angle at the other three sites. The four sites are described by $\phi,-\phi, 90^{\circ}+\phi$, and $90^{\circ}-\phi$.

Figure 4.7 shows the EPR angular dependence data obtained from the $\mathrm{Ti}^{3+}-\mathrm{Si}^{4+}$ center (the complete set of experimental data is presented in Appendix B). The open circles represent experimental data and solid curves are generated by the best set of $g$ parameters. A spin Hamiltonian including only an electron Zeeman term was used to analyze the EPR angular dependence data. Only two crystallographically equivalent sites are needed to describe the angular dependence of the $\mathrm{g}$ matrix for $\mathrm{Ti}^{3+}-\mathrm{Si}^{4+}$ center. These sites are magnetically equivalent when rotating from [010] to [001], and are magnetically inequivalent when rotating in the other two planes. The principal axes of the $g$ matrix are high symmetry directions in the crystal, i.e., the [001], [110], and [1-10] directions. The three principal values of the $g$ matrix are $\mathrm{g}_{[001]}=1.9378, \mathrm{~g}_{[110]}=1.9669$, and $\mathrm{g}_{[1-10]}=$ 1.9162 .

\subsection{Defect Models}

Divalent and trivalent transitions ions $\left(\mathrm{Cu}^{2+}, \mathrm{Fe}^{3+}\right.$, and $\left.\mathrm{Cr}^{3+}\right)$ are common point defects in $\mathrm{TiO}_{2}$ single crystals. They most likely originate with the starting materials (ilmenite ores) and can not be easily removed. Doubly ionized oxygen vacancies (with no trapped electrons) are introduced during growth to charge-compensate these divalent 
or trivalent cations (e.g., the $\mathrm{Fe}^{3+}$ and $\mathrm{Cr}^{3+}$ ions). Oxygen vacancies are effectively double positive charged in an ionic picture of $\mathrm{TiO}_{2}$, and they can trap two electrons to form a local neutral charge state. An oxygen vacancy that traps one electron will form a singly ionized charge state. These trapped electrons are expected to localize on the nearest $\mathrm{Ti}^{4+}$ ions and change their charge state to $\mathrm{Ti}^{3+}$ ions, instead of being localized in the center of the vacancy. There are three nearest-neighbor $\mathrm{Ti}^{4+}$ ions around each oxygen ion. Two of the three $\mathrm{Ti}^{4+}$ ions are along the [001] direction in the crystal, as shown in Fig. 4.8. The distances from the oxygen ion to each of the two $\mathrm{Ti}^{4+}$ ions along [001] direction are the same (1.949 $\AA)$. This is slightly smaller than the distance (1.980 $\AA)$ from the oxygen ion to the third neighboring $\mathrm{Ti}^{4+}$ ion.

I consider three observations when developing the defect models for the $\mathrm{V}_{\mathrm{O}}{ }^{0}$ and the $\mathrm{V}_{\mathrm{O}}{ }^{+}$centers. The first result is that both centers contain paramagnetic $\mathrm{Ti}^{3+}$ ions, as determined by their hyperfine structure. The second result is that the $\mathrm{V}_{\mathrm{O}}{ }^{0}$ center is an $\mathrm{S}=$ 1 system while the $\mathrm{V}_{\mathrm{O}}{ }^{+}$center is an $\mathrm{S}=1 / 2$ system, this requires that the defect can trap either one or two electrons. The third result is that both the $\mathrm{V}_{\mathrm{O}}{ }^{0}$ and the $\mathrm{V}_{\mathrm{O}}{ }^{+}$centers have their unique principal axis along the [001] direction in the crystal. The possible candidates for these defects are interstitial $\mathrm{Ti}^{4+}$ ion and oxygen vacancies. However, interstitial $\mathrm{Ti}^{4+}$ ion is not a common defect in other titanium compounds and is not expected in $\mathrm{TiO}_{2}$ because a $\mathrm{Ti}^{4+}$ ion carries a charge of $4+$, and thus will require nearby charge compensation. An interstitial $\mathrm{Ti}^{4+}$ will also produce a large lattice distortion. When an interstitial $\mathrm{Ti}^{4+}$ traps two electrons, it converts to $\mathrm{Ti}^{2+}$ ion, which is not easily observed by EPR (because of large crystal-field splittings and short lattice-relaxation times). Another possibility is to have the two electrons trapped separately, one on an interstitial $\mathrm{Ti}^{4+}$ ion and one on a neighboring substitutional $\mathrm{Ti}^{4+}$ ion. This results in two adjacent $\mathrm{Ti}^{3+}$ ions. This latter model does not agree with the observation that the unique principal axis of the D matrix is along the [001] direction in the crystal. This then leaves the oxygen vacancy as the only viable model. Oxygen vacancies are common defects in 
(a)

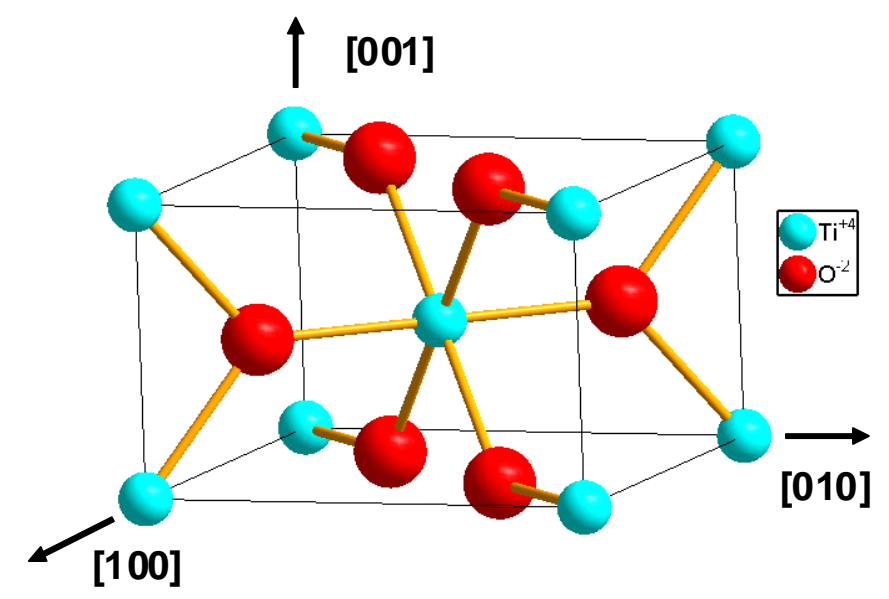

(b)

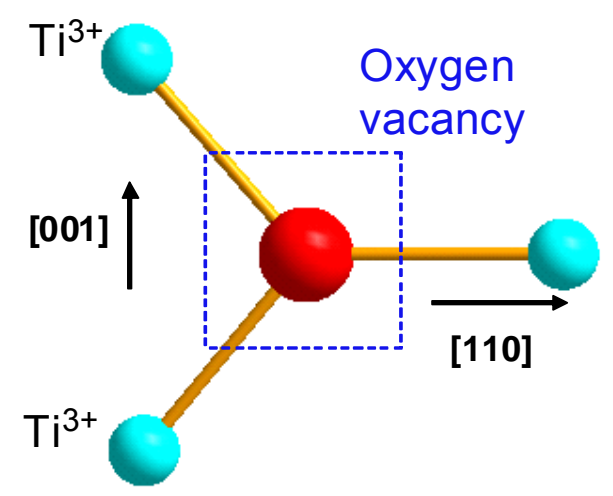

Figure 4.8. Structural model of an oxygen vacancy in a $\mathrm{TiO}_{2}$ crystal. (a) Upper part shows a unit cell of rutile $\mathrm{TiO}_{2}$. (b) Lower part shows the model for the neutral oxygen vacancy.

oxide crystals. A doubly ionized oxygen vacancy (an $\mathrm{S}=0$ defect) can trap one electron and become a singly ionized oxygen vacancy (an $\mathrm{S}=1 / 2 \mathrm{defect}$ ). Alternatively, a doubly ionized oxygen vacancy can trap two electrons and become a neutral oxygen vacancy (an $\mathrm{S}=1$ defect). The simplest explanation, which agrees with my experiments, is to have two electrons localize on the two $\mathrm{Ti}^{4+}$ ions having their internuclear axis along the [001] direction. These two titanium ions have the smallest separation of any pair in the rutile lattice, and they are also closer to the center of the oxygen vacancy than the third 
neighboring $\mathrm{Ti}^{4+}$ ion.

In general, the equilibrium concentration of $\mathrm{V}_{\mathrm{O}}^{0}$ centers produced during an illumination represents a "balance" between the formation and thermal decay rates and thus depends sensitively on both the temperature and the intensity and wavelength of the light. As expected, the $\mathrm{V}_{\mathrm{O}}^{0}$ and $\mathrm{V}_{\mathrm{O}}^{+}$centers were produced in the CrysTec sample at 26 $\mathrm{K}$ with $442 \mathrm{~nm}$ laser light, but their intensities were much lower than in the Namiki sample. This is consistent with EPR results that showed reduced amounts of $\mathrm{Fe}^{3+}$ and $\mathrm{Cr}^{3+}$ ions in the CrysTec sample. In an early study, Kerssen and Volger described $\mathrm{Ti}^{3+}$ centers that have EPR spectra similar to our $\mathrm{V}_{\mathrm{O}}^{+}$and $\mathrm{V}_{\mathrm{O}}^{0}$ centers, and they proposed models for these centers that contained nearby $\mathrm{Al}^{3+}$ ions. Their suggested models are, however, questionable because they did not observe any hyperfine splittings due to the $100 \%$ abundant ${ }^{27} \mathrm{Al}$ nuclei. It is now known [4.11] that an $\mathrm{Al}^{3+}$ ion substituting for a $\mathrm{Ti}^{4+}$ ion will trap a hole on an adjacent oxygen ion, and will not produce electronlike $\mathrm{Ti}^{3+}$ centers.

The mechanism by which below-band-gap light (442 $\mathrm{nm})$ produces trapped electron centers in $\mathrm{TiO}_{2}$ is not fully understood. The most likely scenario involves the direct excitation of electrons from $\mathrm{Fe}^{3+}$ and $\mathrm{Cr}^{3+}$ ions to the conduction band. The $\mathrm{Fe}^{3+}$ and $\mathrm{Cr}^{3+}$ ions, acting as deep singly ionized acceptors, are expected to have near-edge optical transitions (i.e., absorption bands) that correspond to an electron moving from the acceptor to the conduction band. A decrease of about $50 \%$ in the intensities of the $\mathrm{Fe}^{3+}$ and $\mathrm{Cr}^{3+}$ EPR signals occurred when the Namiki sample was illuminated at $26 \mathrm{~K}$ with $442 \mathrm{~nm}$ laser light. This decrease coincided with the production of the $\mathrm{V}_{\mathrm{O}}^{0}$ and $\mathrm{Ti}^{3+}-\mathrm{Si}^{4+}$ centers in Fig. 4.3(a). The trivalent impurity ions convert to $\mathrm{Fe}^{4+}$ and $\mathrm{Cr}^{4+}$ ions and serve as the compensating hole centers for the photoinduced electron centers, thus allowing the crystal to remain electrically neutral. EPR signals from $\mathrm{Fe}^{4+}$ and $\mathrm{Cr}^{4+}$ ions in $\mathrm{TiO}_{2}$ have not been reported. 


\section{Chapter 5}

\section{EPR Study of Self-Trapped Electron and Hole Centers}

\subsection{Introduction}

Oxygen vacancies and $\mathrm{Ti}^{3+}$ ions next to substitutional $\mathrm{Si}^{4+}$ ions were discussed in Chapter 4. In the present chapter, I describe photoinduced EPR experiments that identify self-trapped electron centers and self-trapped hole centers. This work represents an extension of the studies reported in the previous chapter. The experimental techniques and conditions are the same except the measuring temperatures are different. The same $\mathrm{TiO}_{2}$ samples were used in Chapters 4 and 5.

The self-trapped electron center in $\mathrm{TiO}_{2}$ represents an electron that is trapped on a substitutional $\mathrm{Ti}^{4+}$ ion, thus forming a $\mathrm{Ti}^{3+}$ ion in the otherwise perfect lattice. The selftrapped hole center represents a hole trapped on the oxygen sublattice without any nearby defects. Complete sets of spin-Hamiltonian parameters are obtained for the self-trapped electron center and the self-trapped hole center.

\subsection{EPR Spectra of Self-Trapped Centers}

In Chapter 4, I showed that laser light is necessary to populate the paramagnetic charge states of three EPR centers, i.e., the $\mathrm{V}_{\mathrm{O}}^{0}, \mathrm{~V}_{\mathrm{O}}^{+}$, and $\mathrm{Ti}^{3+}-\mathrm{Si}^{4+}$ centers. The spectra of these three centers, taken from the Namiki sample at $26 \mathrm{~K}$, were shown in Fig. 4.3. Two additional $\mathrm{S}=1 / 2 \mathrm{EPR}$ signals were observed when the temperature of the sample was reduced to $18 \mathrm{~K}$ while keeping all the other conditions the same (i.e., the magnetic field along the [001] direction and illuminating with $442 \mathrm{~nm}$ laser light). As shown in Fig. 5.1(a), these two additional $\mathrm{S}=1 / 2$ signals are located at 3345 and $3443 \mathrm{G}$. The signal located at lower field $(3345 \mathrm{G})$ is a holelike center and has a $\mathrm{g}$ value of $\mathrm{g}_{[001]}=$ 2.013. The signal at higher field $(3443 \mathrm{G})$ is an electronlike center and has a $\mathrm{g}$ value of $\mathrm{g}_{[001]}=1.956$. I refer to the center responsible for this electronlike signal as the self- 

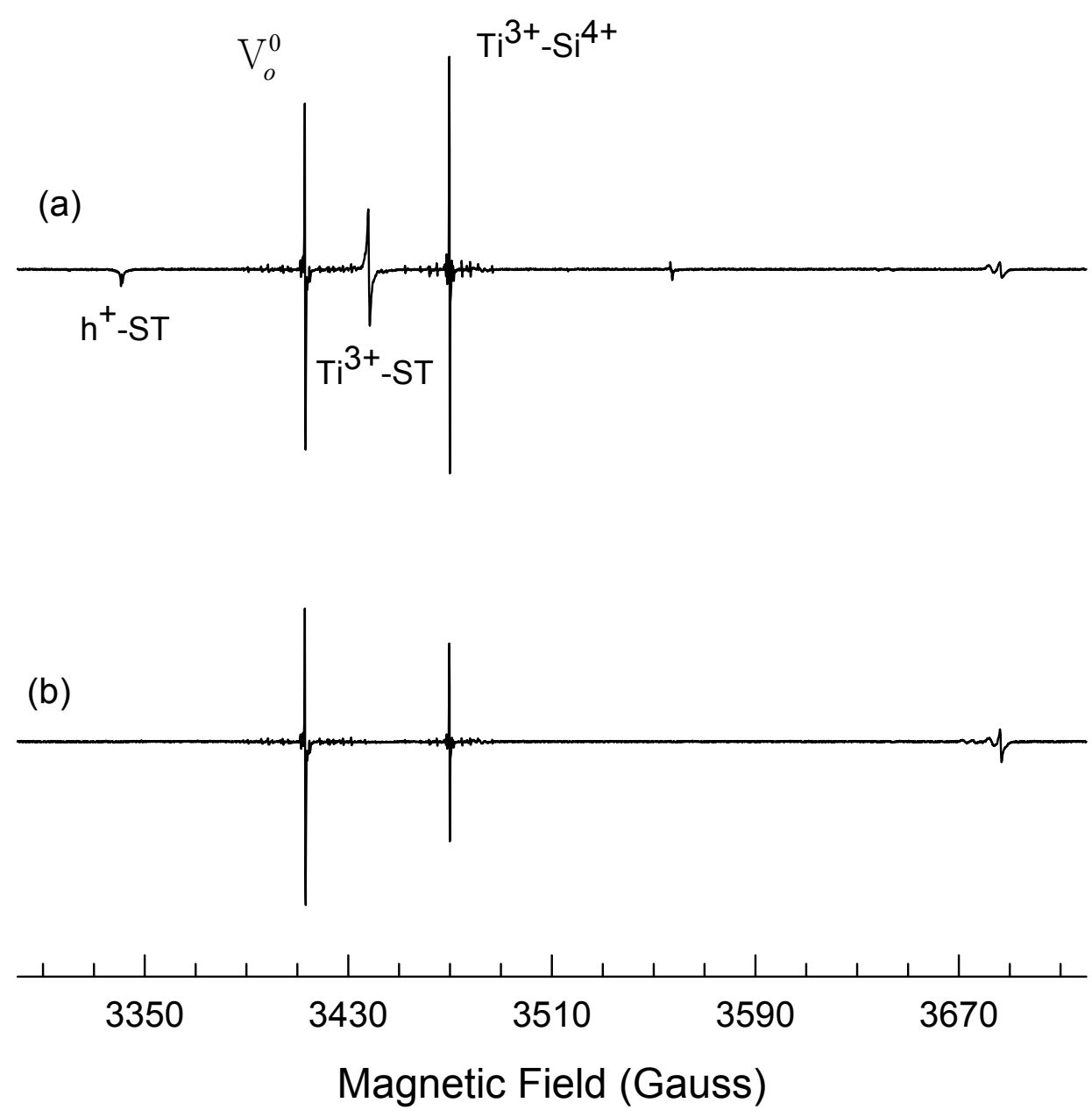

Figure 5.1. EPR spectra of the $\mathrm{STE}\left(\mathrm{Ti}^{3+}\right)$ and $\mathrm{STH}$ centers in $\mathrm{TiO}_{2}$ crystals. These data were taken at $18 \mathrm{~K}$ with the magnetic field parallel to the [001] direction. Trace (a) was taken with the $442 \mathrm{~nm}$ laser light on. Trace (b) was taken after the $442 \mathrm{~nm}$ laser light was removed.

trapped electron center. Similarly, I refer to the center responsible for the holelike signal as the self-trapped hole center. Notations of $\mathrm{STE}\left(\mathrm{Ti}^{3+}\right)$ center and $\mathrm{STH}$ center are used to represent the self-trapped electron center and self-trapped hole center, respectively. Both the STE $\left(\mathrm{Ti}^{3+}\right)$ and the STH centers have very low thermal stabilities. They disappear instantly when the $442 \mathrm{~nm}$ laser light is removed at $18 \mathrm{~K}$, as shown in Fig. 5.1(b). Furthermore, neither the $\mathrm{STE}\left(\mathrm{Ti}^{3+}\right)$ center nor STH center is produced in measurable 


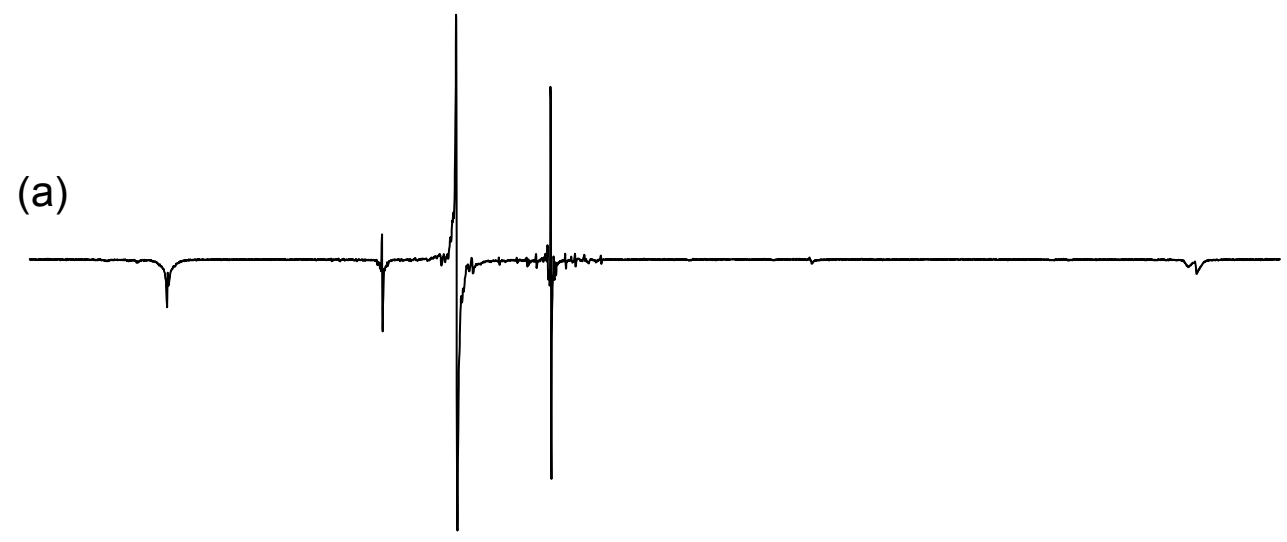

(b)
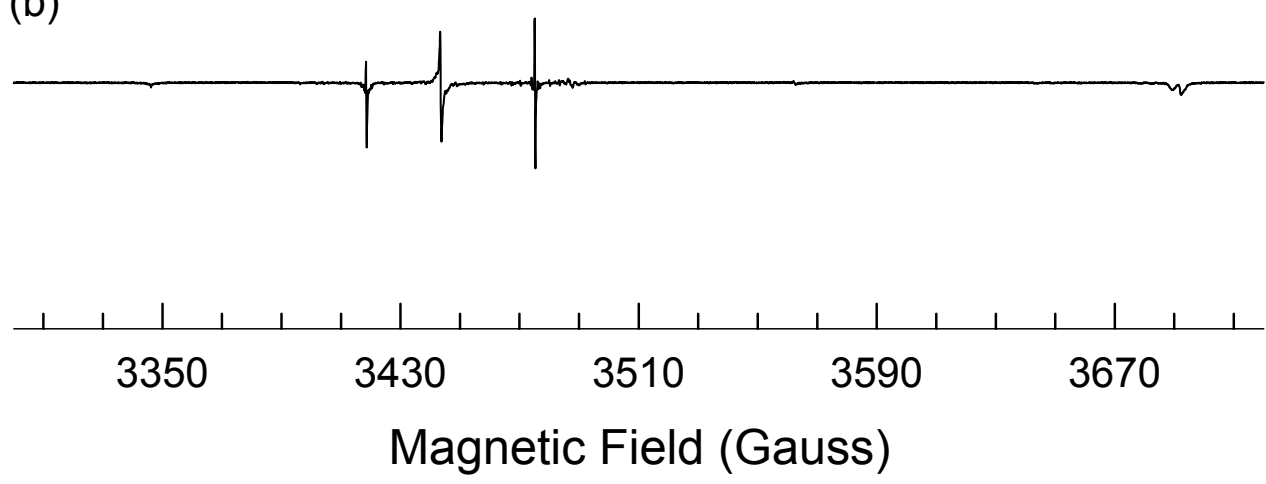

Figure 5.2. EPR spectra of the $\mathrm{STE}\left(\mathrm{Ti}^{3+}\right)$ and $\mathrm{STH}$ centers in $\mathrm{TiO}_{2}$ crystals. These data were taken at $14 \mathrm{~K}$ with the magnetic field parallel to the [001] direction. Trace (a) was taken with the $442 \mathrm{~nm}$ laser light on. Trace (b) was taken after the $442 \mathrm{~nm}$ laser light was removed.

concentrations when the $\mathrm{TiO}_{2}$ sample is illuminated with $442 \mathrm{~nm}$ laser light while the temperature of the sample is above $25 \mathrm{~K}$.

Figure 5.2 shows the EPR spectra of the $\mathrm{STE}\left(\mathrm{Ti}^{3+}\right)$ and STH centers taken at $14 \mathrm{~K}$ with the magnetic field parallel to the [001] direction in the crystal. Figure 5.2(a) was taken during illumination with $442 \mathrm{~nm}$ laser light while Fig. 5.2(b) was taken after the light was removed. At $14 \mathrm{~K}$, the $442 \mathrm{~nm}$ laser light produces EPR signals from these two centers that are much more intense than an illumination at $18 \mathrm{~K}$. These signals are also 
thermally more stable at $14 \mathrm{~K}$ than at $18 \mathrm{~K}$. The EPR signals can still be observed at 14 K several minutes after the $442 \mathrm{~nm}$ laser light is removed (see Fig. 5.2 (b)). The STH and the $\mathrm{STE}\left(\mathrm{Ti}^{3+}\right)$ centers have long spin-lattice relaxation times in the 14 to $25 \mathrm{~K}$ range and were very easily saturated at high microwave powers.

\subsection{Spin-Hamiltonian Analysis}

Figure 5.3 describes the EPR angular dependent data of the STE $\left(\mathrm{Ti}^{3+}\right)$ center (the complete set of experimental data is presented in Appendix B). The horizontal axis represents the direction of the magnetic field, and the vertical axis gives the values of magnetic field where EPR signals occur. The data were taken from three individual planes perpendicular to [010], [1-10] and [001] respectively. The open circles represent experimental data, while the solid curves are generated using the best-fit spinHamiltonian parameters.

Figure 5.4 describes the EPR angular dependence data of the STH centers (the complete set of experimental data is presented in Appendix B). Again, the horizontal axis represents the direction of the magnetic field, and the vertical axis gives the values of magnetic field where EPR signals occur. The data for this center were only taken from two individual planes (from [001] to [110], and from [110] to [1-10]). In the case of the $\mathrm{STE}\left(\mathrm{Ti}^{3+}\right)$ center, angular dependence data were taken in two planes using my sample from Namiki and data in the third plane were taken using a sample from CrysTec. In the case of the STH center, data were not taken in the third plane because the CrysTec sample did not show an easily observed spectrum because of its low concentration and its tendency to saturate at high microwave power. The two planes of data for the STH center show that the defect has the same symmetry as the lattice. Thus, these two planes (from [001] to [110] and from [110] to [1-10]) are sufficient to determine the EPR parameters for this defect. The three principal values of the $g$ matrix correspond to the EPR signal measured at three critical orientations of magnetic field, i.e., one data point 
from the spectrum taken with the magnetic field along the [001] direction and two data points from the spectrum taken with the magnetic field along the [110] direction. Fitting the angular dependence data from two planes (from [001] to [110] and from [110] to [110]) gives the same result as fitting data from three planes. I have verified that this is true for the $\mathrm{Ti}^{3+}-\mathrm{Si}^{4+}$ center and the $\operatorname{STE}\left(\mathrm{Ti}^{3+}\right)$ center.

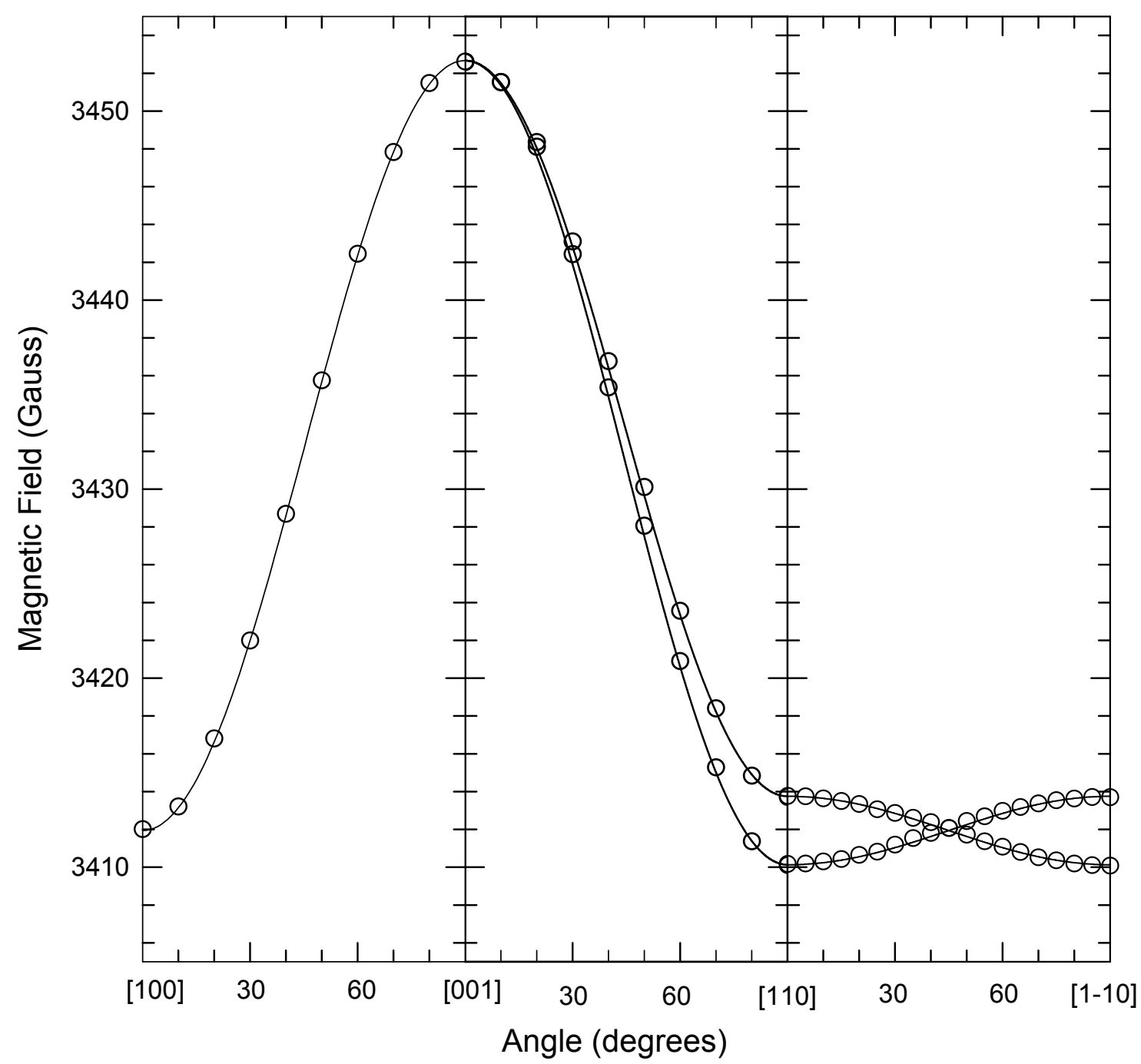

Figure 5.3. EPR angular dependence data of $\mathrm{STE}\left(\mathrm{Ti}^{3+}\right) \mathrm{ST}$ centers. The discrete open circles are experimental data, while the solid curves are computer generated using the best-fit $g$ matrix. 
The following spin Hamiltonian, with an electron Zeeman term only, was used to separately analyze the angular dependence data from the STE $\left(\mathrm{Ti}^{3+}\right)$ and STH centers.

$$
\mathbf{H}=\beta \mathbf{S} \cdot \mathbf{g} \cdot \mathbf{B}
$$

A least-squares fitting program written in MATLAB was employed to generate the bestfit parameters (i.e., the g matrix). The resulting best-fit parameters for the $\operatorname{STE}\left(\mathrm{Ti}^{3+}\right)$ and

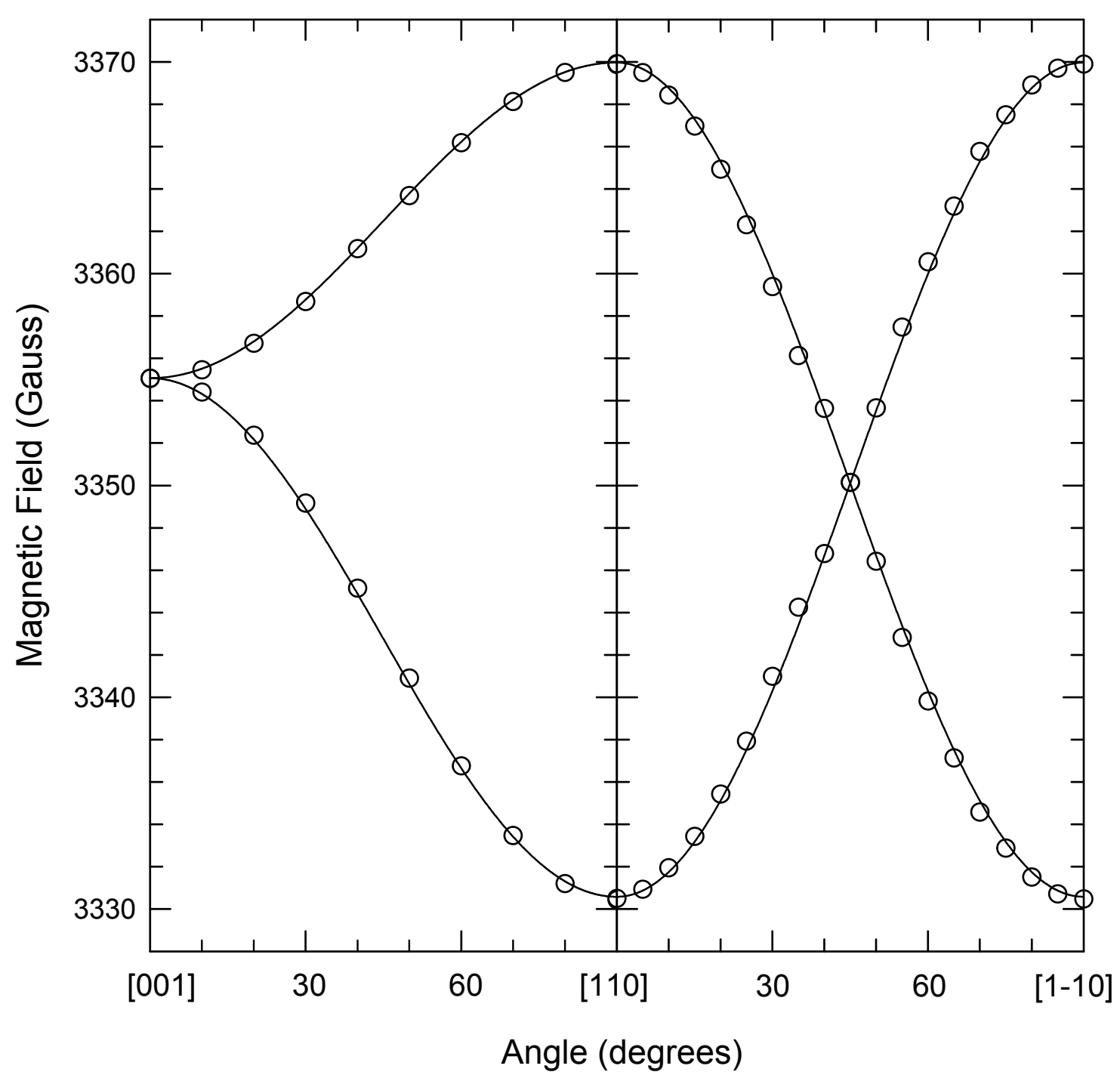

Figure 5.4. EPR angular dependence data of STH centers. The discrete open circles are experimental data, while the solid curves are computer generated using the best-fit g matrix. 
STH centers were used to generate the solid curves in Figs. 5.3 and 5.4, and they are listed in Table 5.1.

Table 5.1. Spin-Hamiltonian parameters for the self-trapped electron center and the self-trapped hole center.

\begin{tabular}{|c|c|c|}
\hline \multirow{2}{*}{ Center } & \multicolumn{2}{|c|}{ Principal Values } \\
\hline \multirow{3}{*}{ STE $\left(\mathrm{Ti}^{3+}\right)$} & $\mathrm{g}_{110}$ & 1.9783 \\
\cline { 2 - 3 } & $\mathrm{g}_{1-10}$ & 1.9804 \\
\cline { 2 - 3 } & $\mathrm{g}_{001}$ & 1.9560 \\
\hline \multirow{3}{*}{ STH } & $\mathrm{g}_{110}$ & 2.0277 \\
\cline { 2 - 3 } & $\mathrm{g}_{1-10}$ & 2.0040 \\
\cline { 2 - 3 } & $\mathrm{g}_{001}$ & 2.0129 \\
\hline
\end{tabular}

\subsection{Defect Models}

The STE $\left(\mathrm{Ti}^{3+}\right)$ center and the STH center were the dominant photoinduced EPR signals in the CrysTec sample with $0.5 \mathrm{~mm}$ thickness (see Chapter 4 for sample description) when it was illuminated with $442 \mathrm{~nm}$ laser light while being held at $14 \mathrm{~K}$. The two oxygen vacancy centers (i.e., the neutral and the singly ionized oxygen vacancies) were much less intense in the CrysTec sample during low temperature illuminations because the crystal contained fewer oxygen vacancies. Based on their low thermal stabilities and their appearance in both the Namiki and the CrysTec samples, I propose models involving the self trapping of photoinduced charges for the EPR signals at 3345 and $3443 \mathrm{G}$. The holelike signal has no resolved hyperfine structure, which indicates that there is no nearby trivalent cation impurity that serves to "stabilize" the hole. This leads me to assign the EPR signal at $3345 \mathrm{G}$ in $\mathrm{TiO}_{2}$ to a "self-trapped" hole, i.e., a hole localized on one oxygen ion or shared by two adjacent oxygen ions that have 
relaxed toward each other. In the later case, the two neighboring oxygen ions could have their internuclear axis along the [001] direction in the crystal. Thus, the self-trapped hole defect has the same symmetry as the crystal. An analogous self-trapped hole defect has been observed in $\mathrm{KD}_{2} \mathrm{PO}_{4}$ crystals [5.1]. Similarly, I assign the signal at $3443 \mathrm{G}$ to a "self-trapped" electron in the form of an isolated $\mathrm{Ti}^{3+}$ ion, i.e., an electron trapped at a $\mathrm{Ti}^{4+}$ ion in the otherwise perfect lattice.

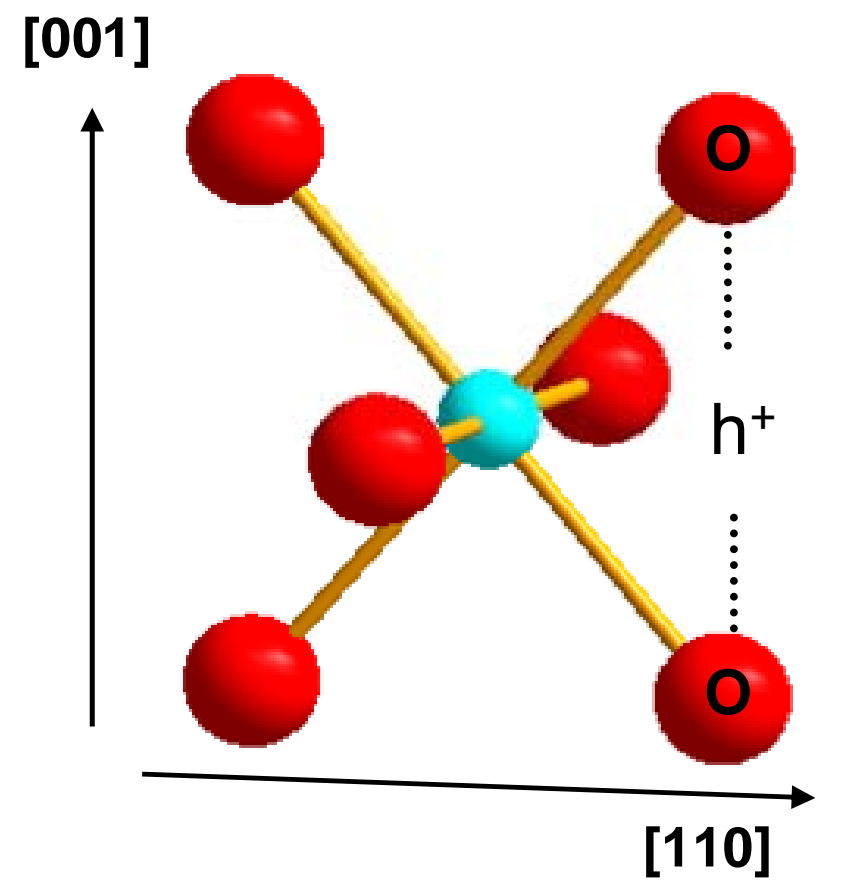

Figure 5.5. Defect model for the $\mathrm{STH}$ center in $\mathrm{TiO}_{2}$ crystals. The hole may be trapped on one oxygen ion or shared by two oxygen ions. 


\section{Chapter 6}

\section{Fluorine Donors and $\mathrm{Ti}^{3+}$ Ions in $\mathrm{TiO}_{2}$ Crystals}

\subsection{Introduction}

In my early experimental study of the photoinduced paramagnetic charge states of oxygen vacancies in $\mathrm{TiO}_{2}$ (rutile) crystals, I showed an important result that the ground state of the neutral oxygen vacancy is magnetic (i.e., the neutral oxygen vacancy has $\mathrm{S}=$ 1) [6.1-6.3]. The two electrons trapped at the vacancy are localized on two of the three neighboring titanium ions and are coupled ferromagnetically [6.3]. These observations may help explain the ferromagnetic behavior reported in recent studies of $\mathrm{TiO}_{2}$ [6.4-6.6].

In this chapter, EPR and ENDOR are used to determine the electronic structure of the neutral fluorine donor in single crystals of $\mathrm{TiO}_{2}$. Fluorine ions substitute for oxygen ions in this lattice [6.7-6.10] and act as shallow donors. In a fully oxidized sample, these isolated fluorine donors are initially in the singly ionized charge state (with no unpaired electrons to give an EPR signal). At low temperature, $442 \mathrm{~nm}$ laser light populates the neutral charge state (thus producing an $\mathrm{S}=1 / 2 \mathrm{EPR}$ signal). This EPR signal in a fully oxidized sample quickly decays at low temperature after removing the light when selftrapped holes thermally release and recombine with the electrons at the neutral fluorine donors. In contrast, the neutral fluorine donor EPR signal is present at low temperature without photoexcitation in a sample that has been reduced at high temperature. The principal values and directions of the principal axes for the $\mathrm{g}$ matrix and the ${ }^{19} \mathrm{~F}$ hyperfine matrix are obtained from the angular dependences of the EPR and ENDOR spectra. A model consisting of a substitutional $\mathrm{Ti}^{3+}$ ion adjacent to a substitutional $\mathrm{F}^{-}$ion is established for the ground state of the neutral fluorine donor in $\mathrm{TiO}_{2}$, i.e., the unpaired spin is localized primarily on a neighboring titanium ion instead of being centered on the fluorine ion. 


\subsection{Experimental Details}

The $\mathrm{TiO}_{2}$ crystals used in this investigation have the rutile structure and were purchased from MTI Corporation (Richmond, CA). They were grown by the floatingzone method. Three EPR-sized samples for this study, one with dimensions of $3 \times 5 \times 1$ $\mathrm{mm}^{3}$ and two with dimensions of $2 \times 2 \times 1 \mathrm{~mm}^{3}$, were cut from a larger [001] plate $(10 \mathrm{x}$ $10 \times 1 \mathrm{~mm}^{3}$ ) provided by MTI. Fluorine was present in all the crystals supplied by MTI and, to my knowledge, was an unintentional contaminant. Its concentration level in my crystals, estimated from EPR, was less than one ppm by weight. The crystals received from MTI were colorless at room temperature and only exhibited EPR signals from $\mathrm{Fe}^{3+}$ and $\mathrm{Cr}^{3+}$ ions, thus indicating that they were fully oxidized. Subsequent reducing treatments in our laboratory consisted of placing a sample in flowing nitrogen gas while heating to high temperature.

\subsection{EPR Results}

Figure 6.1 shows the EPR doublet produced at $6 \mathrm{~K}$ in my fully oxidized $3 \times 5 \times 1$ $\mathrm{mm}^{3} \mathrm{TiO}_{2}$ sample during exposure to $442 \mathrm{~nm}$ laser light. These spectra were taken with the magnetic field along the [001] direction. There were no EPR signals in this magnetic field region before illumination, as shown in Fig. 6.1(a). The photoinduced EPR doublet in Fig. 6.1(b) has a splitting of $1.80 \mathrm{G}$ and linewidths of $0.45 \mathrm{G}$ for this orientation of magnetic field. ENDOR experiments, described in Section 6.4, identify a hyperfine interaction with a ${ }^{19} \mathrm{~F}$ nucleus $(\mathrm{I}=1 / 2,100 \%$ abundant $)$ as the cause of the splitting. This, in turn, leads me to assign the EPR signal in Fig. 6.1(b) to a neutral fluorine donor.

I determined, with EPR, that trace amounts of $\mathrm{Fe}^{3+}$ and $\mathrm{Cr}^{3+}$ ions substitute for $\mathrm{Ti}^{4+}$ ions in my samples [6.11-6.13]. These trivalent transition-metal ions act as deep singly ionized acceptors and provide compensation for the singly ionized fluorine donors in the as-received crystals (i.e., one trivalent transition-metal ion charge compensates one $\mathrm{F}^{-}$ion replacing an oxygen ion). Additional electronlike EPR signals appear in my fully 
(a)
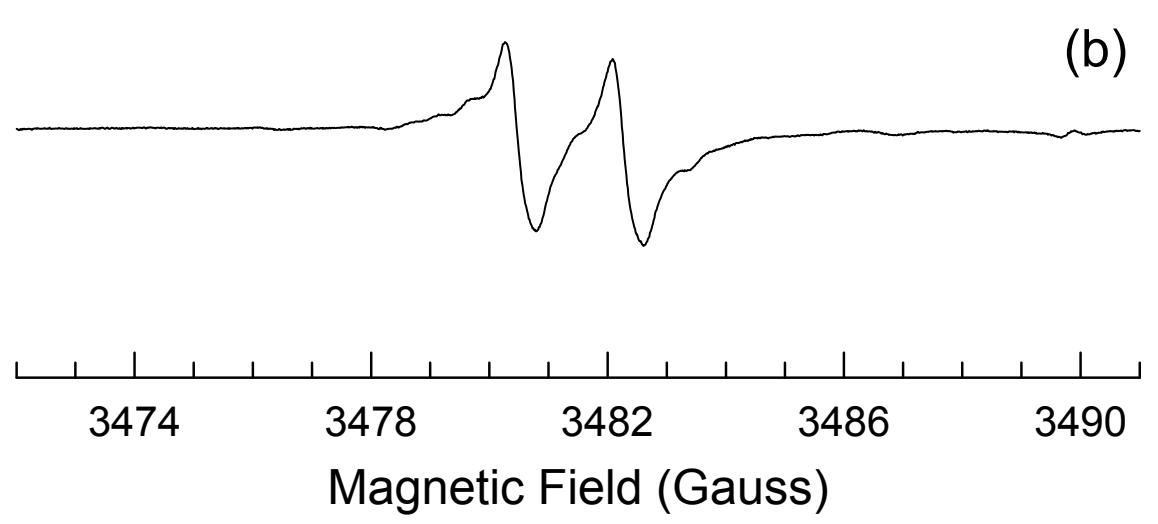

Figure 6.1. EPR spectrum of the photoinduced neutral fluorine donor in $\mathrm{TiO}_{2}$ (rutile). These data were taken at $6 \mathrm{~K}$ with the magnetic field along the [001] direction. (a) Upper spectrum was taken in the dark after cooling in the dark. (b) Lower spectrum was taken during exposure to $442 \mathrm{~nm}$ laser light.

oxidized samples during illumination at low temperature, including singly ionized oxygen vacancies, neutral oxygen vacancies, $\mathrm{Ti}^{3+}-\mathrm{Si}^{4+}$ centers, and $\mathrm{Ti}^{3+}$ self-trapped electrons [6.3]. Significant concentrations of self-trapped holes are also produced during illumination [6.3]. All of these EPR signals are easily saturated with microwave power at temperatures below $15 \mathrm{~K}$, and thus, although present, are not readily observed under the conditions used to obtain the spectra in Fig. 6.1. Self-trapped hole centers in $\mathrm{TiO}_{2}$ (rutile) consist of a hole localized on one or two oxygen ions in an otherwise perfect region of the crystal with lattice relaxation providing the trapping potential [6.3]. These selftrapped hole centers are important in the present study as they are the source of the photoexcited electrons that temporarily convert the singly ionized fluorine donors to their 


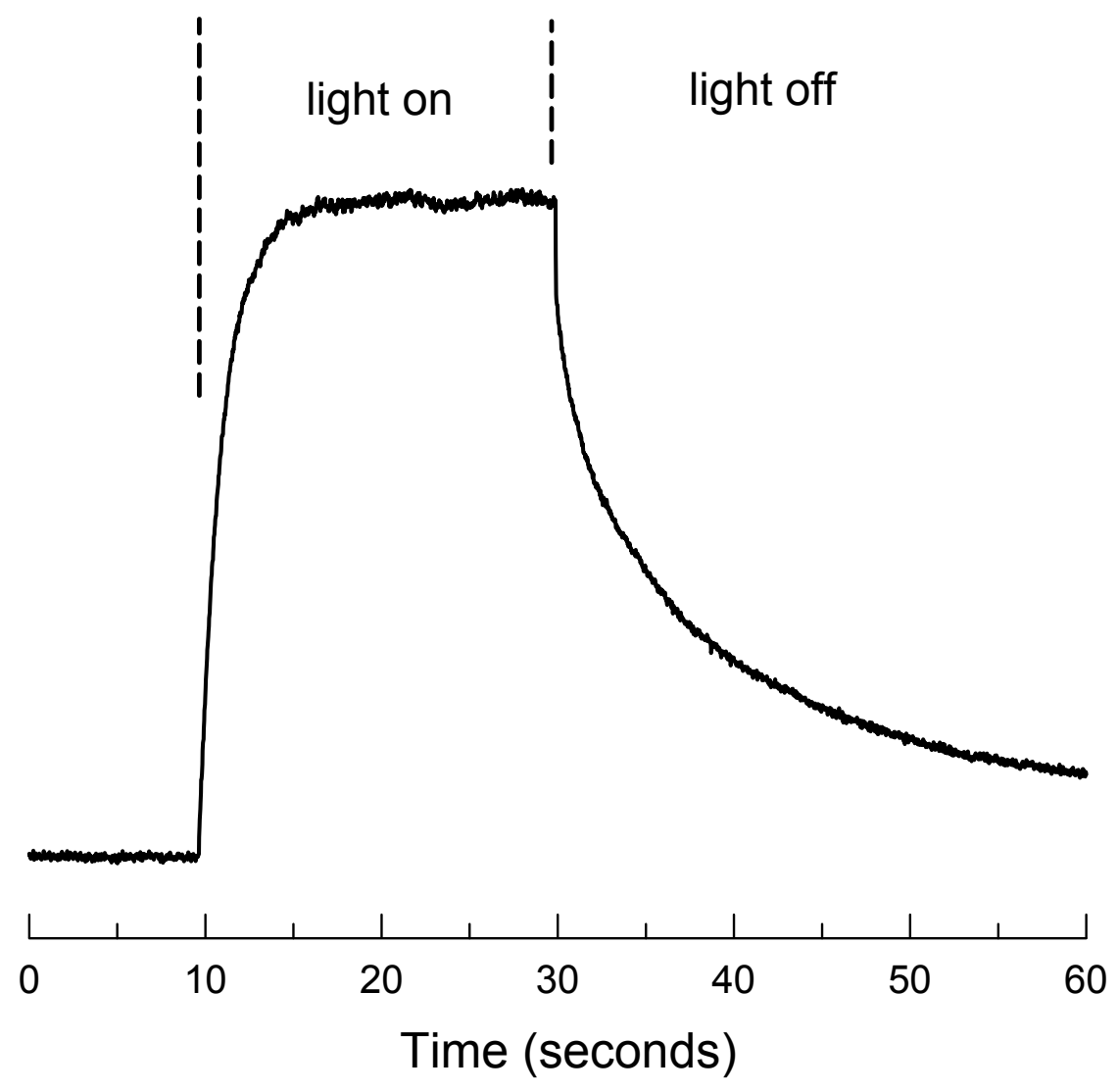

Figure 6.2. Production and decay of the neutral fluorine donor EPR spectrum as the $442 \mathrm{~nm}$ laser light was turned on and off. The monitoring temperature was $4.6 \mathrm{~K}$.

neutral charge state at the very low temperatures. Monitoring the $\mathrm{Fe}^{3+}$ and $\mathrm{Cr}^{3+} \mathrm{EPR}$ signals shows that they are not the primary source of electrons to form neutral fluorine donors when the temperature during illumination is below $15 \mathrm{~K}$. These transition-metal ions are expected, however, to be critical participants in the mechanism that allows below-band-gap light (442 nm) to form neutral fluorine donors in my crystals.

The production and thermal decay of the neutral fluorine donor in my fully oxidized $3 \times 5 \times 1 \mathrm{~mm}^{3}$ sample is illustrated in Fig. 6.2. These data were acquired by fixing the magnetic field at the peak of the derivative for one of the components of the fluorine doublet (see Fig. 6.1) and then monitoring the intensity of this EPR signal as a 

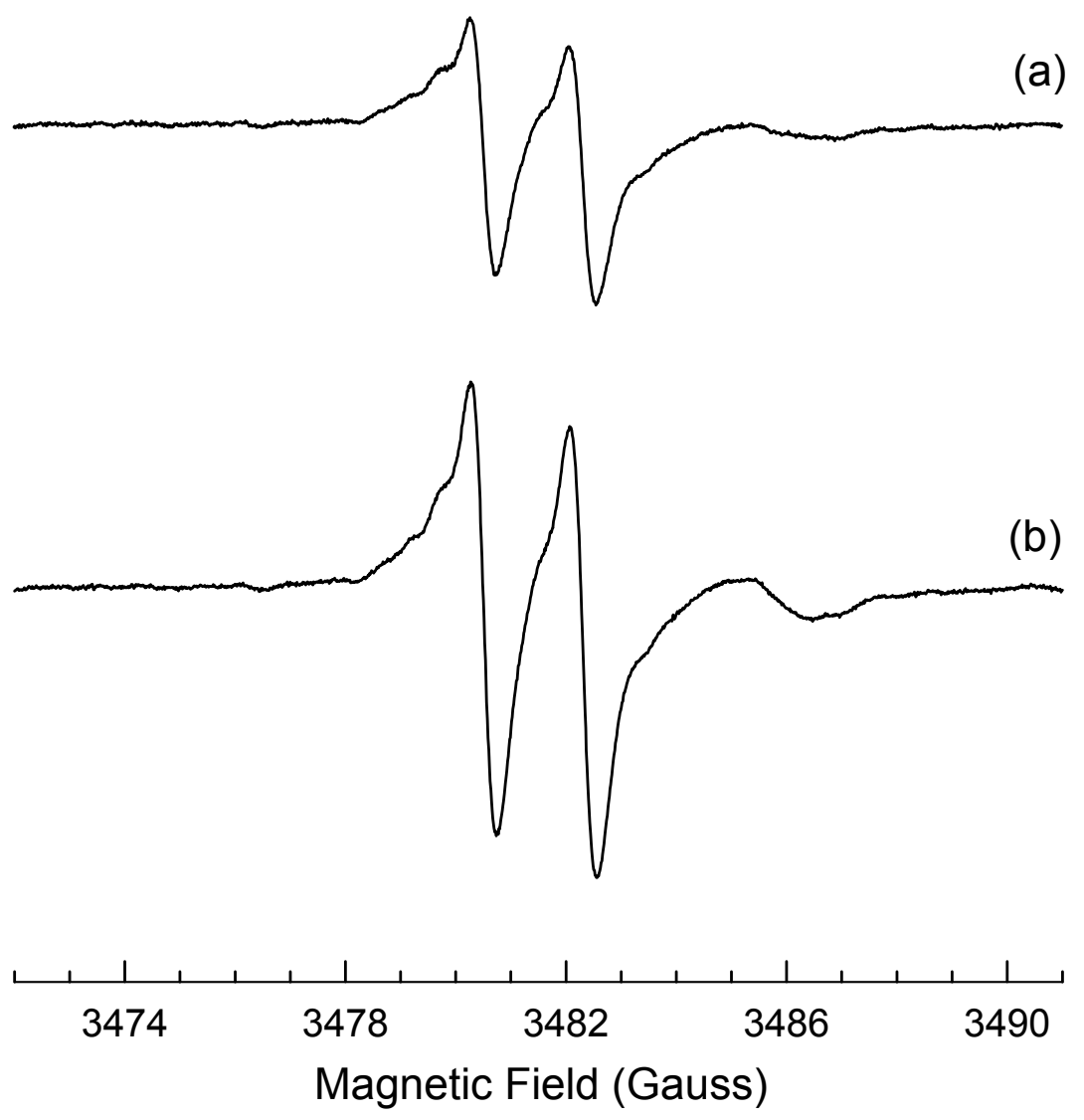

Figure 6.3. EPR spectra taken at $6 \mathrm{~K}$ after reducing a $\mathrm{TiO}_{2}$ crystal for 90 minutes at $600{ }^{\circ} \mathrm{C}$ in flowing nitrogen gas. The magnetic field was along the [001] direction. (a) Spectrum taken in the dark after cooling in the dark. (b) Spectrum taken during subsequent exposure to $442 \mathrm{~nm}$ laser light.

function of time while the $442 \mathrm{~nm}$ laser light was turned on and off. During this experiment, the temperature was maintained near $4.6 \mathrm{~K}$ and the magnetic field was along the [001] direction. The EPR signal reaches saturation within 5 seconds after the laser is turned on (the production rate depends on the incident laser power and the temperature). At $4.6 \mathrm{~K}$, the EPR signal decays over tens of seconds when the laser is turned off and the sample is held in the dark. Raising the temperature slightly results in a faster decay of the EPR signal. At $7 \mathrm{~K}$, the photoinduced EPR signal drops to a barely detectable level 
within a few seconds when the laser light is removed. I suggest that the rapid decay of the EPR signal in Fig. 6.2 occurs when holes are thermally released from the photoinduced self-trapped hole centers [6.3]. These thermally released holes move to the neutral fluorine donors and recombine with the trapped electrons, thus converting the neutral fluorine donors back to their singly ionized state. This release of holes in the dark at temperatures below $15 \mathrm{~K}$ is not a property of the fluorine donors in $\mathrm{TiO}_{2}$, and does not provide information about the ionization energy of these donors. Instead, the rapid decay of the neutral fluorine EPR signal at very low temperature indicates that the ionization energy of the self-trapped hole center is about 10 to $15 \mathrm{meV}$.

Following the characterization of the neutral fluorine EPR signals in the fully oxidized sample, one of the two smaller samples cut from the larger as-received $\mathrm{TiO}_{2}$ plate was held at $600{ }^{\circ} \mathrm{C}$ for 90 minutes in flowing $\mathrm{N}_{2}$ gas and then placed in the EPR spectrometer. The EPR signal from the neutral fluorine donor was easily observed in this reduced sample even though there was no light incident on the crystal during cooling or at low temperature prior to or during the time the spectrum was taken. These data are shown in Fig. 6.3(a) where the measuring temperature is $6 \mathrm{~K}$ and the magnetic field is along the [001] direction. The EPR signal became more intense when this sample was illuminated with $442 \mathrm{~nm}$ laser light while at $6 \mathrm{~K}$, as shown in Fig. 6.3(b). A similar reducing treatment of the second smaller sample at $650{ }^{\circ} \mathrm{C}$ resulted in an even more intense fluorine EPR signal without light, and there was no increase in the EPR signal when the sample was exposed to $442 \mathrm{~nm}$ light at $6 \mathrm{~K}$. In general, heating in a nitrogen atmosphere introduces shallow donors (i.e., neutral oxygen vacancies), thus making the $\mathrm{TiO}_{2}$ crystal more $\mathrm{n}$ type and significantly increasing the number of free carriers. At the low temperatures where I take EPR data, a portion of these electrons "freeze out" at the fluorine sites and produce the neutral fluorine donors that I observe with EPR in the absence of light. In other words, the reducing treatment raises the Fermi level and populates the neutral fluorine donors. An interesting feature in Fig. 6.3 is the weak EPR 


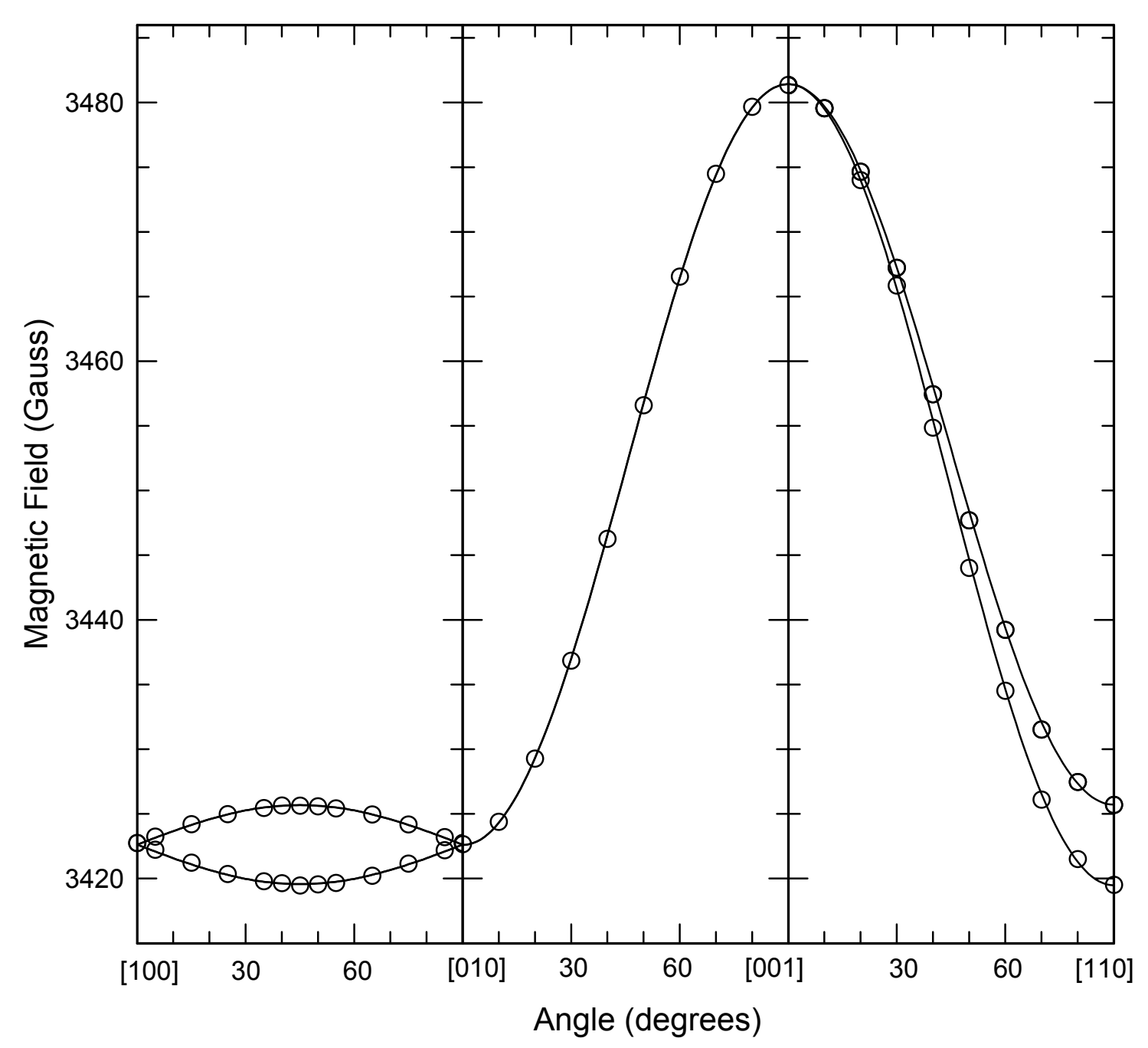

Figure 6.4. Angular dependence of the g matrix for the neutral fluorine donor in $\mathrm{TiO}_{2}$. These EPR data were acquired in the three high-symmetry planes of the crystal. Single points at the centers of the doublets are used for those angles where the fluorine hyperfine is resolved in the EPR spectra.

signal near $3486 \mathrm{G}$ (corresponding to an effective $\mathrm{g}$ value of 1.9408 when the magnetic field is parallel to the [001] direction). I also observe this EPR line in $\mathrm{TiO}_{2}$ crystals that do not contain detectable amounts of fluorine donors. ENDOR spectra taken in our laboratory show that this EPR line at $\mathrm{g}=1.9408$ when the magnetic field is along the 
[001] direction has an unresolved hyperfine interaction with one hydrogen ( $I=1 / 2$, nearly $100 \%$ abundant) [6.14]. I suggest that the responsible defect is a $\mathrm{Ti}^{3+}$ ion adjacent to an $\mathrm{OH}^{-}$molecular ion (i.e., a neutral hydrogen donor in rutile-structured $\mathrm{TiO}_{2}$ ). A complete angular dependence of the neutral fluorine donor EPR spectrum was taken at $6 \mathrm{~K}$ using the sample reduced at $600{ }^{\circ} \mathrm{C}$ for 90 minutes. As shown in Fig. 6.4, data were acquired in all three high-symmetry planes of the crystal (the complete set of experimental data is presented in Appendix B). The sample was illuminated with $442 \mathrm{~nm}$ light during the measurements. These plots provide information about the principal values of the $g$

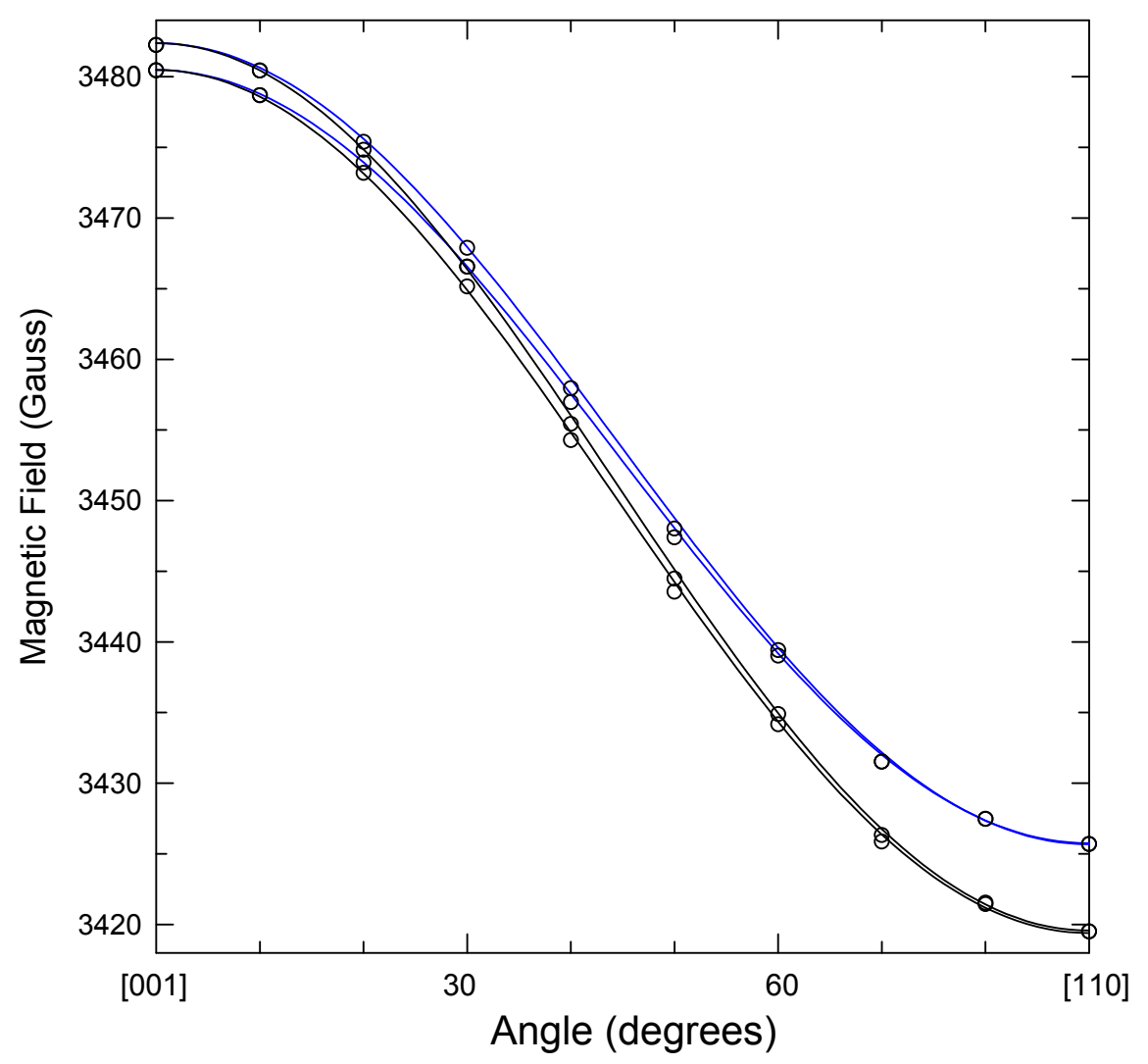

Figure 6.5. Angular dependence of the g matrix for the neutral fluorine donor in $\mathrm{TiO}_{2}$. These EPR data were acquired in the plane including [001] to [110] directions in the crystal. Open circles are data points and solid line are generated using best fitting g matrix. Blue and black indicate two different sites. 
matrix and the directions of the principal axes. The discrete points in Fig. 6.4 are experimental results and the solid curves are computer-generated using the final set of "best" parameters. Fluorine hyperfine splittings are resolved in the EPR spectra for orientations of the magnetic field within approximately $60^{\circ}$ of the [001] direction. At these angles, I have plotted the center positions of the resolved doublets. Thus, there is no hyperfine information in Fig. 6.4. In order to illustrate hyperfine splitting versus site splitting, a detailed view of the hyperfine splittings (i.e., angular dependence) for the plane of rotation from the [001] direction to the [110] direction is shown in Fig. 6.5. The pair of EPR lines occurring in the [001] spectrum is caused by a hyperfine interaction; whereas the pair of EPR lines in the [110] spectrum is caused by site splitting, which is a result of the two distinguishable octahedrons. The two sites are magnetically equivalent when the magnetic field is parallel to the [001] direction of the crystal, and nonequivalent when the magnetic field is parallel to the [110] direction of the crystal. I note that the site with low-field EPR lines during the rotation from [001] to [110] in Fig. 6.5 has the larger fluorine hyperfine splitting.

The following spin-Hamiltonian, with only an electron Zeeman term, was used to determine the parameters describing the angular dependence data in Fig. 6.4.

$$
\mathbf{H}=\beta \mathbf{S} \cdot \mathbf{g} \cdot \mathbf{B}
$$

Only two crystallographically equivalent sites are needed to describe the angular dependence of the g matrix. These sites are magnetically equivalent when rotating from [010] to [001], and are magnetically inequivalent when rotating in the other two planes in Fig. 6.4. The two sites are associated with the two equivalent distorted $\mathrm{TiO}_{6}$ octahedra that are present in the $\mathrm{TiO}_{2}$ (rutile) lattice. These $\mathrm{TiO}_{6}$ units are elongated in directions perpendicular to the [001] direction with the six oxygen ions separating into a set of two along the elongation direction and a set of four perpendicular to the elongation direction. The two octahedra are related by a $90^{\circ}$ rotation about the [001] direction. Figure 6.4 
shows that the directions of the principal axes of the g matrix for the fluorine donor are along high-symmetry directions in the crystal. Principal values of 1.9746, 1.9782, and 1.9430 are obtained from the magnetic fields and microwave frequencies measured at the turning points in Fig. 6.4, i.e., the two turning points along [110] and the one turning point along [001]. The principal axis corresponding to the 1.9430 principal value is along the [001] direction in the crystal. My ENDOR results in Section 6.4 show that the principal axis associated with the 1.9746 principal value is along the elongation direction containing the two equivalent oxygen ions in $\mathrm{TiO}_{6}$ octahedron. I specify this elongation direction as [110] for this octahedron. The principal values and the directions of the principal axes for the g matrix at one site are summarized in Table 6.1.

\subsection{ENDOR Results}

The [001] ENDOR spectrum of the neutral fluorine donor is shown in Fig. 6.6. These data were taken at $5 \mathrm{~K}$ from the sample that had been reduced at $650{ }^{\circ} \mathrm{C}$. This choice of sample eliminated the need to use the $442 \mathrm{~nm}$ laser light at low temperature to maximize the fluorine donor EPR signal. The two lines in Fig. 6.6 are separated by 4.915 MHz and are centered on $13.986 \mathrm{MHz}$. Their linewidths are $55 \mathrm{kHz}$. For a weak hyperfine interaction, the first-order ENDOR spectrum from an $\mathrm{I}=1 / 2$ nucleus consists of two lines separated by the hyperfine parameter A and centered on the "free" nuclear spin frequency $v_{N}\left(\right.$ where $\left.v_{N}=g_{N} \beta_{N} H / h\right)$. The ENDOR spectrum in Fig. 6.6 was taken at a magnetic field of $3487.24 \mathrm{G}$, and the corresponding value of $v_{\mathrm{N}}$ for a ${ }^{19} \mathrm{~F}$ nucleus is 13.976 MHz (values of $v_{\mathrm{N}}$ for all nuclei are tabulated in Appendix A of Reference [6.2]). This known value of $v_{\mathrm{N}}$ for ${ }^{19} \mathrm{~F}$ is very close to the experimental value for the center of the two lines in Fig. 6.6, and thus conclusively shows that a ${ }^{19} \mathrm{~F}$ nucleus is responsible for the hyperfine splitting observed in the [001] EPR spectrum.

Figure 6.7 shows the angular dependence of the ${ }^{19} \mathrm{~F}$ ENDOR spectrum. Results from all three high-symmetry planes are included (the complete set of experimental data 


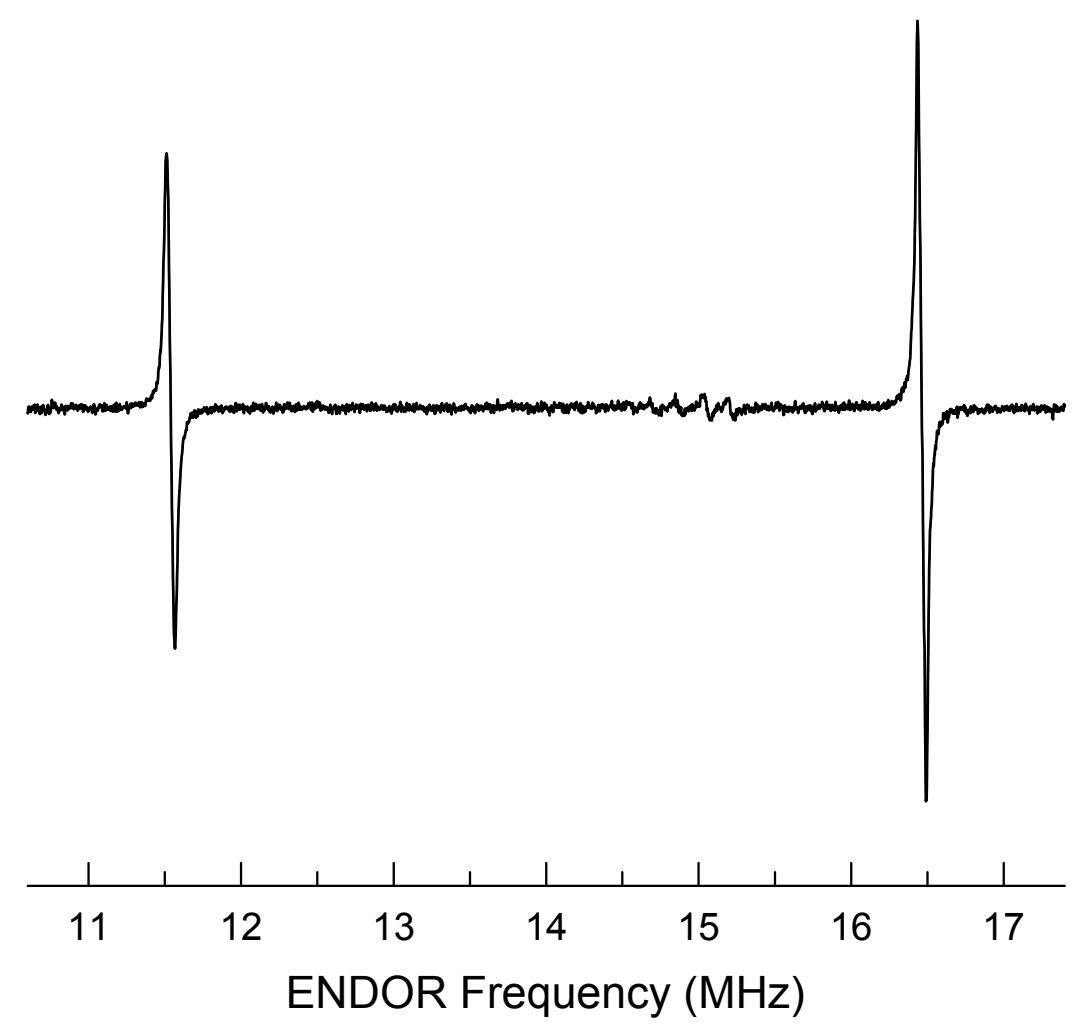

Figure 6.6. ENDOR spectrum from the neutral fluorine donor in the $\mathrm{TiO}_{2}$ crystal, taken at $5 \mathrm{~K}$ with the magnetic field parallel to the [001] direction.

is presented in Appendix B). The discrete points are experimental data taken at $5 \mathrm{~K}$ with no illumination and the solid curves are computer-generated using the final set of "best" parameters. Four crystallographically equivalent sites are needed to describe the ${ }^{19} \mathrm{~F}$ hyperfine matrix. Two of these sites are associated with one distorted $\mathrm{TiO}_{6}$ octahedron and two are associated with the second distorted $\mathrm{TiO}_{6}$ octahedron generated from the first by a $90^{\circ}$ rotation about the [001] direction. As shown in Fig. 6.7, the four sites are pairwise magnetically equivalent when the magnetic field is rotated from [100] to [010] and from [010] to [001]. The four sites divide into three sets (with degeneracies of one, two, and one) when rotating the magnetic field from [001] to [110]. Along the [110] direction of magnetic field in Fig. 6.7, the inner pair of ENDOR lines at 13.68 and 13.89 


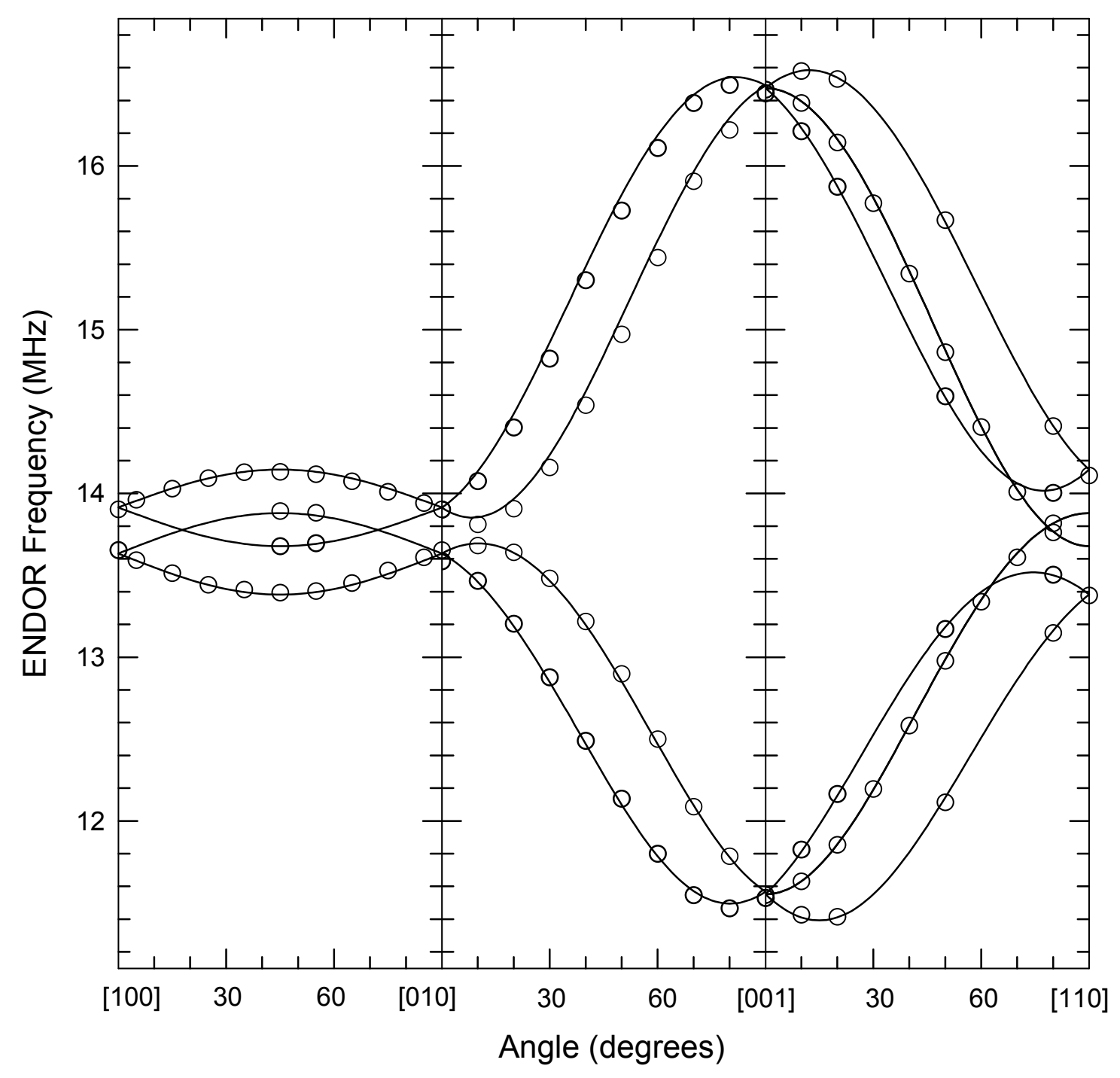

Figure 6.7. Angular dependence of the hyperfine matrix for the neutral fluorine donor in $\mathrm{TiO}_{2}$. These ENDOR data were acquired in the three high-symmetry planes of the crystal.

$\mathrm{MHz}$ are obtained from the EPR line at $3440.1 \mathrm{G}$ while the outer pair of ENDOR lines at 13.38 and $14.14 \mathrm{MHz}$ are obtained from the EPR line at $3433.9 \mathrm{G}$. This inner pair of ENDOR lines comes from the two sites that are magnetically equivalent when the magnetic field is rotated from [001] to [110]. 
Table 6.1. Spin-Hamiltonian parameters for the neutral fluorine donor in a $\mathrm{TiO}_{2}$ (rutile) crystal. The [110] principal axes of $g_{1}$ and $\mathrm{A}_{1}$ are arbitrarily defined to be parallel to the elongation direction of a distorted $\mathrm{TiO}_{6}$ octahedron (see Section 6.3). Directions of the principal axes for $A_{2}$ and $A_{3}$ are in the (110) plane (see the model in Fig. 6.8).

\begin{tabular}{lcc}
\hline & Principal Value & Principal-Axis Direction \\
\hline g matrix & $1.9746 \pm 0.0001$ & {$[110]$} \\
$\mathrm{g}_{1}$ & $1.9782 \pm 0.0001$ & {$[\overline{1} 10]$} \\
$\mathrm{g}_{2}$ & $1.9430 \pm 0.0001$ & {$[001]$} \\
$\mathrm{g}_{3}$ & $-0.23 \mathrm{MHz} \pm 0.01 \mathrm{MHz}$ & {$[110]$} \\
${ }^{19} \mathrm{~F}$ hyperfine matrix & $13.8^{\circ}$ from [ $\left.\overline{1} 10\right]$ \\
$\mathrm{A}_{1}$ & $0.47 \mathrm{MHz} \pm 0.01 \mathrm{MHz}$ & $13.8^{\circ}$ from [001] \\
$\mathrm{A}_{2}$ & $5.15 \mathrm{MHz} \pm 0.01 \mathrm{MHz}$ & \\
$\mathrm{A}_{3}$ & & \\
\hline
\end{tabular}

The following spin-Hamiltonian, with electron Zeeman, hyperfine, and nuclear Zeeman terms, was used to analyze the angular data in Fig. 6.7.

$$
\mathbf{H}=\beta \mathbf{S} \cdot \mathbf{g} \cdot \mathbf{B}+\mathbf{I} \cdot \mathbf{A} \cdot \mathbf{S}-\mathrm{g}_{\mathrm{N}} \beta_{\mathrm{N}} \mathbf{I} \cdot \mathbf{B}
$$

Final values for the hyperfine parameters were obtained from a least squares fitting procedure that involved exact diagonalizations of the $4 \times 4$ Hamiltonian matrix $(S=1 / 2$, I $=1 / 2$ ). When fitting the ENDOR data, the $g$ matrix was fixed at the values and directions given in Table 6.1. From the data in Fig. 6.7, one of the principal axes of the ${ }^{19} \mathrm{~F}$ hyperfine matrix must be along the [110] direction. Specifically, I find that the 1.9746 principal $g$ value and the $-0.23 \mathrm{MHz}$ principal hyperfine value have principal axes along 
[001]
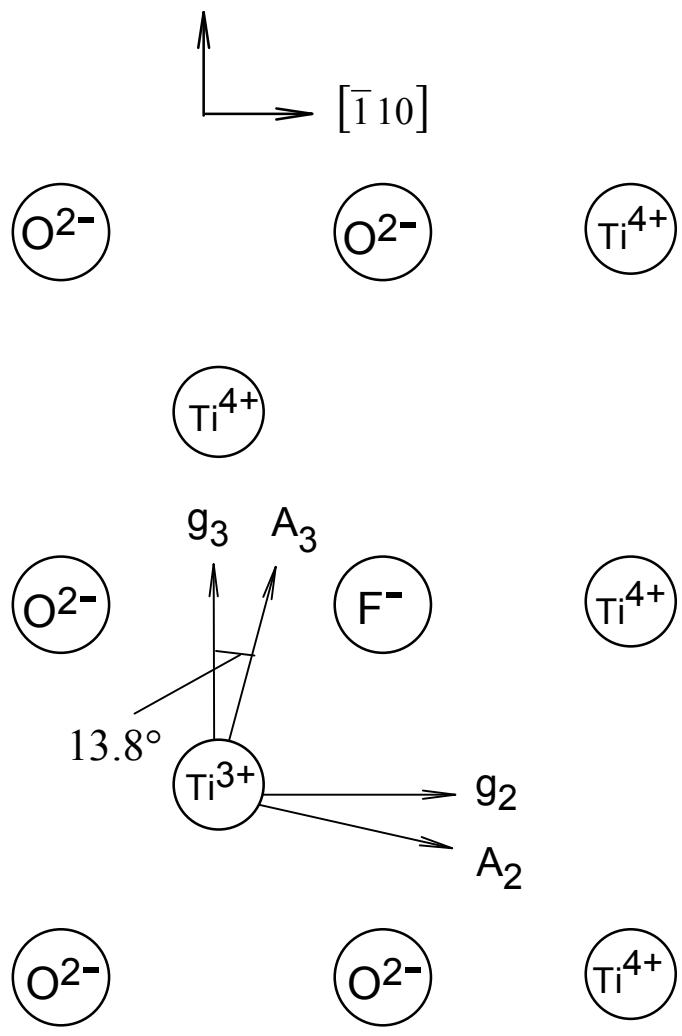

Figure 6.8. Model of the neutral fluorine donor illustrating the localization of the unpaired electron on the neighboring titanium ion. This is a projection on the (110) plane. The $g_{1}$ and $A_{1}$ principal axes are along the [110] direction.

the [110] direction (i.e., the elongation direction containing the two equivalent oxygen ions in a distorted $\mathrm{TiO}_{6}$ octahedron). Turning points in Fig. 6.7 indicate that the remaining two hyperfine principal axes are not along high-symmetry directions of the crystal. These principal axes must, however, be in the (110) plane, and one angle suffices to define their directions. Thus, during the ENDOR fitting process, only four parameters were varied (three principal values and one angle). Results obtained from fitting the experimental data in Fig. 6.7 are listed for one site in Table 6.1. 


\subsection{Discussion}

Fluorine ions substitute for oxygen ions in $\mathrm{TiO}_{2}$ (rutile) and form shallow donors. All of the fluorine donors are in the singly ionized charge state (with no unpaired spins, and thus no EPR signal) in fully oxidized crystals cooled in the dark when a sufficient number of transition-metal-ion acceptors are present to provide compensation.

Photoexcited electrons produced during an illumination at very low temperature convert a portion of these fluorine donors to the neutral charge state which is EPR-active.

However, unlike a classic donor, the unpaired electron associated with the neutral charge state of the fluorine donor is not centered on the fluorine ion in a hydrogenlike wave function that spreads out over many shells of neighboring ions and reflects the symmetry of the lattice. Instead, I find that the unpaired electron is localized, to a first approximation, on one titanium ion immediately adjacent to the fluorine ion. In other words, it is energetically more favorable to have the "extra" electron in a d orbital on the titanium ion than delocalized in an effective-mass (i.e., hydrogenlike) wave function centered on the fluorine ion.

My primary evidence for a $\mathrm{Ti}^{3+}$ model for the neutral fluorine donor comes from the fluorine hyperfine matrix. Table 6.1 shows that the ${ }^{19} \mathrm{~F}$ matrix has significant anisotropy, with the principal axis associated with the largest principal value $(5.15 \mathrm{MHz})$ deviating $13.8^{\circ}$ from the [001] direction. If a hydrogenic model for the fluorine donor were correct, then all of the principal axes of the hyperfine matrix would be along highsymmetry directions in the crystal and the anisotropy would be minimal. A model for the neutral fluorine donor is shown in Fig. 6.8, including principal axes of the $\mathrm{g}$ and ${ }^{19} \mathrm{~F}$ hyperfine matrices. A substitutional fluorine ion in $\mathrm{TiO}_{2}$ has three nearest-neighbor titanium ions. The two neighboring titanium ions along the [001] direction are equivalent, and they are closer to the fluorine ion than the remaining titanium ion along the $[\overline{1} 10]$ direction. In the regular $\mathrm{TiO}_{2}$ lattice at room temperature, these Ti-O distances are 
1.9485 and $1.9800 \AA$, respectively [6.15]. As I show in Fig. 6.8, the neutral fluorine donor has the electron localized on one of these two titanium ions along the [001] direction. The Coulomb energy is minimized in this configuration. This Coulomb energy would be higher if the electron was localized on the slightly more distant titanium ion along the $[\overline{1} 10]$ direction. Also, the ${ }^{19} \mathrm{~F}$ hyperfine matrix would not exhibit the measured $13.8^{\circ}$ angle if the electron was localized on the titanium ion in the [ $\left.\overline{1} 10\right]$ direction.

The measured g matrix is consistent with my model of a substitutional $\mathrm{Ti}^{3+}$ ion next to an $\mathrm{F}^{-}$ion. Figure 6.9(a) shows one of the two equivalent distorted $\mathrm{TiO}_{6}$ octahedra in the $\mathrm{TiO}_{2}$ (rutile) lattice. $\mathrm{The}_{\mathrm{Ti}}{ }^{3+}$ ion is at the center and the six neighboring oxygens are represented by the dark solid circles. I consider the case when six oxygen ions are present (replacing one of the four equivalent oxygen ions in the (110) plane with a fluorine does not change my analysis). The $x, y, z$ coordinate system in Fig. 6.9(a) has $x$ along the $[\overline{1} 10]$ direction, $\mathrm{y}$ along the [001] direction, and $\mathrm{z}$ along the [110] direction. As shown in Appendix A, the five d orbitals can be written as:

$$
\begin{aligned}
& |x y\rangle=-\frac{i}{\sqrt{2}}(|2,2\rangle-|2,-2\rangle) \\
& |x z\rangle=\frac{1}{\sqrt{2}}(|2,-1\rangle-|2,1\rangle) \\
& |y z\rangle=\frac{i}{\sqrt{2}}(|2,-1\rangle+|2,1\rangle) \\
& \left|x^{2}-y^{2}\right\rangle=\frac{1}{\sqrt{2}}(|2,2\rangle+|2,-2\rangle) \\
& \left|z^{2}\right\rangle=|2,0\rangle
\end{aligned}
$$

Note that the $\mathrm{x}$ and $\mathrm{y}$ axes do not point toward the nearest-neighbor negative ions in Fig. 6.9(a). The choice of coordinate system and the positions of the nearest-neighbor negative ions in Fig. 6.9(a) produce the relative energy ordering shown in Fig. 6.9(b) for these five $d$ orbitals $\left(\left|x^{2}-y^{2}\right\rangle\right.$ is lowest in energy and $\left|z^{2}\right\rangle$ is highest in energy). As described by Kasai, [6.16] the $\left|\mathrm{x}^{2}-\mathrm{y}^{2}\right\rangle$ orbital is lowest in energy because of two nearest- 


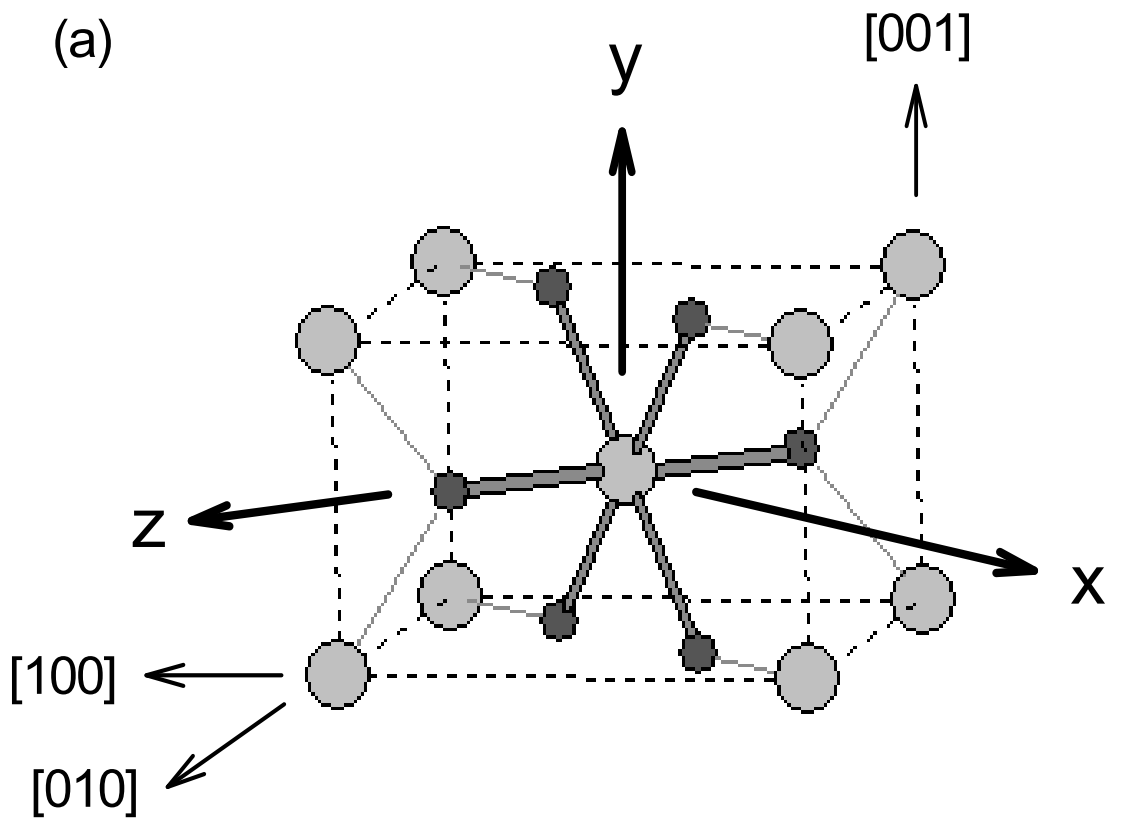

(b)

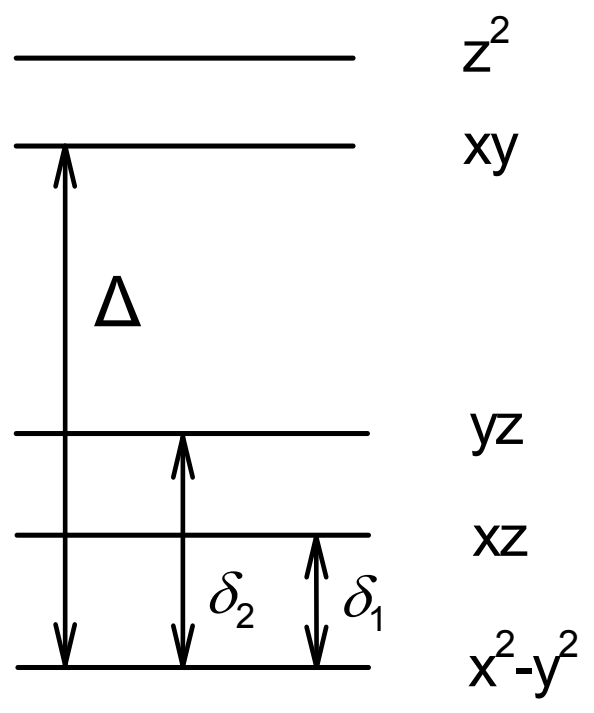

Figure 6.9. (a) Distorted $\mathrm{TiO}_{6}$ octahedron (the center ion is the $\mathrm{Ti}^{3+}$ ion, the six smaller dark solid circles are oxygen ions, and the eight larger shaded circles are $\mathrm{Ti}^{4+}$ ions). The $\mathrm{x}, \mathrm{y}, \mathrm{z}$ coordinate system is used in the g matrix analysis. (b) Energy ordering of the five d orbitals. 
neighbor $\mathrm{Ti}^{4+}$ ions along the [001] direction, one above and one below the $\mathrm{Ti}^{3+}$ ion. The g matrix for a $\mathrm{d}^{1}$ electron is then given, to first order, by the following expressions [6.17]:

$$
\mathbf{g}=\mathrm{g}_{\mathrm{e}} \hat{\mathbf{i}}+2 \lambda \hat{\Lambda} \quad \text { where } \quad \Lambda_{\mathrm{ij}}=-\sum_{\mathrm{n} \neq \mathrm{G}} \frac{\left\langle\mathrm{G}\left|\mathrm{L}_{\mathrm{i}}\right| \mathrm{n}\right\rangle\left\langle\mathrm{n}\left|\mathrm{L}_{\mathrm{j}}\right| \mathrm{G}\right\rangle}{\mathrm{E}_{\mathrm{n}}-\mathrm{E}_{\mathrm{G}}}
$$

From these expressions (as shown in Appendix A), I arrive at the principal values of the $g$ matrix.

$$
g_{x x}=g_{e}-\frac{2 \lambda}{\delta_{2}}, \quad g_{y y}=g_{e}-\frac{2 \lambda}{\delta_{1}}, \quad g_{z z}=g_{e}-\frac{8 \lambda}{\Delta}
$$

The energy differences $\delta_{1}, \delta_{2}$, and $\Delta$ are defined in Fig. 6.9(b). In Eq. (6.9), $\mathrm{g}_{\mathrm{e}}=2.0023$ and $\lambda$ is the spin-orbit coupling constant $\left(154 \mathrm{~cm}^{-1}\right.$ for a $\mathrm{Ti}^{3+}$ ion). To partially account for covalency, I include an orbital reduction factor $\mathrm{k}$ that reduces the spin-orbit constant $\left(\lambda^{\prime}=\mathrm{k} \lambda\right)$. I take $\mathrm{k}$ to be 0.6 , thus making $\lambda^{\prime}=92.4 \mathrm{~cm}^{-1}$. The measured $\mathrm{g}_{1}, \mathrm{~g}_{2}$, and $\mathrm{g}_{3}$ values in Table I correspond to $g_{z z}, g_{x x}$, and $g_{y y}$, respectively. Substituting these measured $\mathrm{g}$ values and my values for $\mathrm{k}$ and $\lambda$ into Eq. (6.9) gives $\delta_{1}=3120 \mathrm{~cm}^{-1}, \delta_{2}=$

$7670 \mathrm{~cm}^{-1}$, and $\Delta=26700 \mathrm{~cm}^{-1}$. A cursory examination of the measured principal $\mathrm{g}$ values in Table 6.1 suggests that the g matrix is very nearly axial. My analysis, however, verifies the orthorhombic nature of a titanium site and shows that a fortuitous splitting of the $\mathrm{d}$ levels causes $\mathrm{g}_{1}$ and $\mathrm{g}_{2}$ to have similar values.

\subsection{Summary}

A detailed EPR and ENDOR study of the neutral fluorine donor in $\mathrm{TiO}_{2}$ (rutile) bulk crystals is reported. These neutral donors, along with self-trapped hole centers, are produced in fully oxidized crystals by illumination with below-band-gap laser light (442 $\mathrm{nm})$ at low temperature ( $10 \mathrm{~K}$ and below). The EPR signals from these defects quickly decay at the low temperatures when the light is removed, as holes thermally release from the self-trapped hole centers and recombine with electrons trapped at the fluorine ions. 
Reducing a $\mathrm{TiO}_{2}$ crystal in a nitrogen atmosphere introduces neutral oxygen-vacancy donors that raise the Fermi level and populate the neutral fluorine donors. In the reduced crystal, electrons are available from these oxygen vacancies to form the neutral fluorine donors, and photoexcitation is not needed. From the angular dependences of the EPR and ENDOR spectra, I determine that the neutral fluorine donor in $\mathrm{TiO}_{2}$ consists of a substitutional $\mathrm{Ti}^{3+}$ ion adjacent to a $\mathrm{F}^{-}$ion substituting for an oxygen ion. My analysis of the principal values of the $g$ matrix shows that the unpaired $\mathrm{d}$ electron on the $\mathrm{Ti}^{3+}$ ion occupies a $\left|x^{2}-y^{2}\right\rangle$ orbital. 


\section{Chapter 7}

\section{Hydrogen Donors and $\mathrm{Ti}^{3+}$ Ions in $\mathrm{TiO}_{2}$ Crystals}

\subsection{Introduction}

Hydrogen is a common impurity in oxide crystals such as $\mathrm{ZnO}$ and $\mathrm{MgO}$ [7.1]. In semiconductor materials, hydrogen often acts as a shallow donor [7.2, 7.3]. Infrared absorption studies have shown the existence of hydrogen in $\mathrm{TiO}_{2}$ single crystals $[7.1,7.4]$. Understanding the influence of hydrogen on the electronic structure of $\mathrm{TiO}_{2}$ crystals may result in improvements in their applications. EPR and ENDOR are suitable techniques to identify and characterize point defects such as hydrogen. Until now, there have been no EPR signals assigned to hydrogen in $\mathrm{TiO}_{2}$. In this chapter, an EPR signal is assigned to the neutral hydrogen donor in $\mathrm{TiO}_{2}$ (rutile) crystals, and the assignment is verified in ENDOR experiments. EPR signals with almost the same g values have been reported in earlier papers by different groups $[7.5,7.6]$, but those investigators did not know that hydrogen was part of the defect. I suggest that their spectra represent the same hydrogen donor signal identified in my studies. My results now allow their signals to be interpreted correctly.

\subsection{Experimental Details}

In this chapter, I describe experimental results from four $\mathrm{TiO}_{2}$ samples, including two as-received and two reduced samples. The first as-received sample, with dimensions $2.5 \times 3.5 \times 1.2 \mathrm{~mm}^{3}$, was obtained from Namiki and was used for the oxygen-vacancy studies in Chapter 4. The second as-received sample, with dimensions $2.5 \times 3.5 \times 2.0$ $\mathrm{mm}^{3}$, was obtained from CrysTec. The two reduced samples, with dimensions of $2.0 \mathrm{x}$ $3.0 \times 0.5 \mathrm{~mm}^{3}$ and $2.0 \times 2.0 \times 1.0 \mathrm{~mm}^{3}$, were from CrysTec and MTI, respectively.

The two reduced samples were fully oxidized when originally received. Reducing $\mathrm{TiO}_{2}$ generally means heating the sample to high temperature in an oxygen deficient 
atmosphere. In my reducing process, the samples are heated in a quartz tube while $\mathrm{N}_{2}$ gas flows through it. The long quartz tube containing the $\mathrm{TiO}_{2}$ sample is placed in a furnace, and its two ends sticking out of the furnace are sealed with two silicon rubber stoppers. Both rubber stoppers have a small hole at the center, where a smaller-diameter quartz tube was inserted. The quartz tube is purged with $\mathrm{N}_{2}$ gas for about 5 minutes before the furnace was turned on to remove the air inside it. It took 20 minutes for the furnace to reach its set temperature (usually between 650 and $700{ }^{\circ} \mathrm{C}$ ). The sample was kept at this temperature for times ranging from 30 to 90 minutes. The quartz tube was then removed from the furnace quickly with the $\mathrm{N}_{2}$ gas continuing to flow. This rapidly cooled the sample to room temperature.

The CrysTec sample with $0.5 \mathrm{~mm}$ thickness was annealed for 30 minutes at 700 ${ }^{\circ} \mathrm{C}$. The MTI sample was annealed for 60 minutes at $600{ }^{\circ} \mathrm{C}$ and then annealed for an additional 50 minutes at $650{ }^{\circ} \mathrm{C}$. I refer to these two samples as the $700{ }^{\circ} \mathrm{C}$ annealed CrysTec sample and the $650{ }^{\circ} \mathrm{C}$ annealed MTI sample in this chapter. The two samples that were not reduced are referred to as the as-received Namiki sample and the asreceived CrysTec sample.

\subsection{EPR Results}

All four samples show trace amounts of $\mathrm{Fe}^{3+}$ and $\mathrm{Cr}^{3+}$ impurities which are charge compensated by oxygen vacancies. Figure 7.1 shows EPR spectra taken from the asreceived CrysTec sample. These spectra were taken at $4 \mathrm{~K}$, with the magnetic field parallel to the [001] direction in the crystal. The upper trace (a) was taken before any illumination; no EPR signals were observed in the region shown in Fig. 7.1. In contrast, the lower trace (b) was taken after exposing the sample to $442 \mathrm{~nm}$ laser light while at $4 \mathrm{~K}$; a dominant sharp EPR signal was observed. The effective $g$ value for this signal is $g_{[001]}$ $=1.9408$. I refer to this signal as the neutral hydrogen donor. My ENDOR data shows that a hydrogen nucleus $(I=1 / 2,99.985 \%$ abundance $)$ participates in this EPR signal. 
(a)

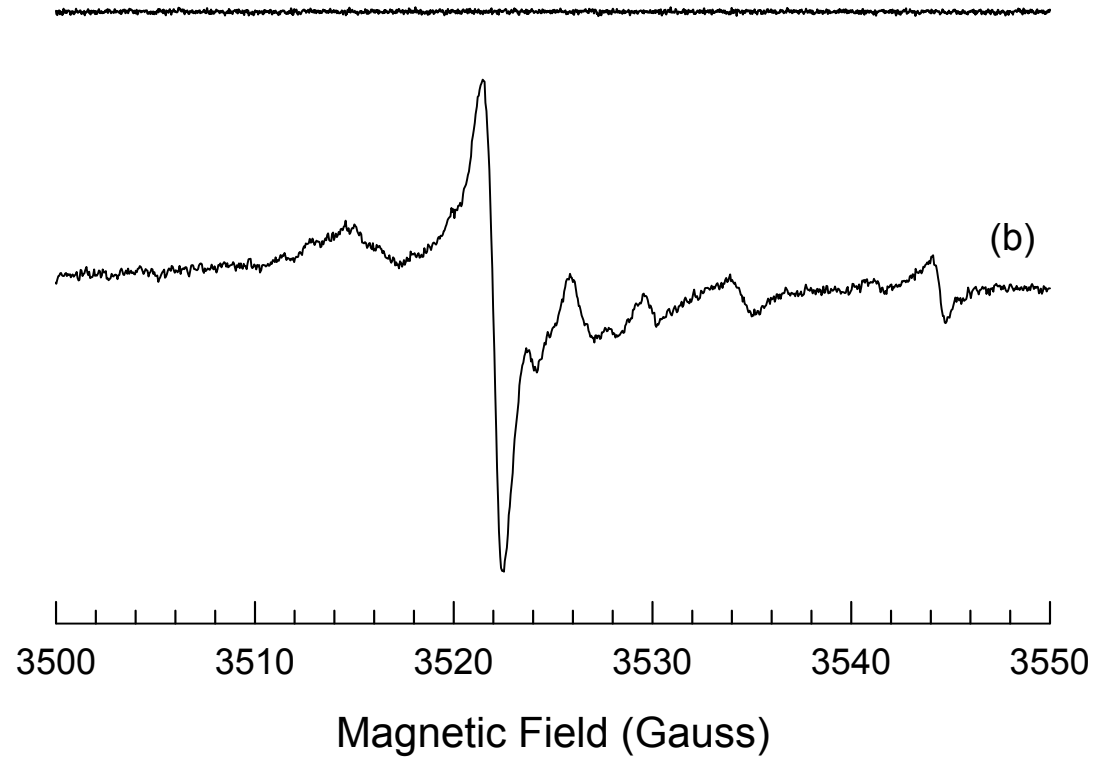

Figure 7.1. The EPR spectrum of the neutral hydrogen donor in an as-received CrysTec $\mathrm{TiO}_{2}$ crystal. The data were taken at $4 \mathrm{~K}$ with the magnetic field along the [001] direction. (a) Without illumination. (b) During illumination with 442 nm laser light.

(a)

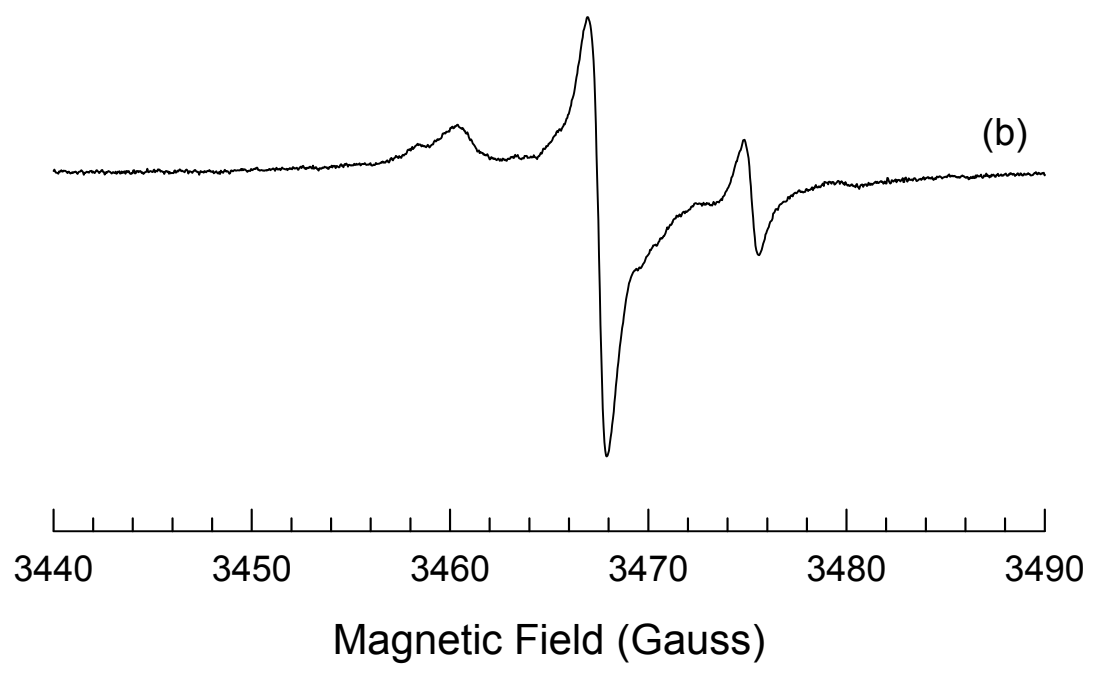

Figure 7.2. The EPR spectrum of the neutral hydrogen donor in an as-received Namiki $\mathrm{TiO}_{2}$ crystal. The data were taken at $4 \mathrm{~K}$ with the magnetic field along the [001] direction. (a) Without illumination. (b) During illumination with 442 $\mathrm{nm}$ laser light. 
The hydrogen donor EPR signal is not stable at this low temperature and disappears within a few minutes at $4 \mathrm{~K}$ when the laser light is removed. The signal can not be observed above $8 \mathrm{~K}$ during illumination with the $442 \mathrm{~nm}$ laser light. Figure 7.2 shows the similar result from the as-received Namiki sample. Several additional small EPR signals appeared in both samples, and they are not identified at this moment. I note that the magnetic field values for the neutral hydrogen donor signal are slightly shifted in Figs. 7.1 and 7.2 for the CrysTec and the Namiki samples. This field difference is caused by the change in microwave frequency that occurs when different-sized samples are put in the EPR cavity. $\mathrm{TiO}_{2}$ has a large dielectric constant $(\sim 170$ at $300 \mathrm{~K})$, thus its volume and shape will significantly affect the frequency of the microwave cavity.

Figure 7.3 shows the EPR signal taken from the CrysTec sample after 30 minutes

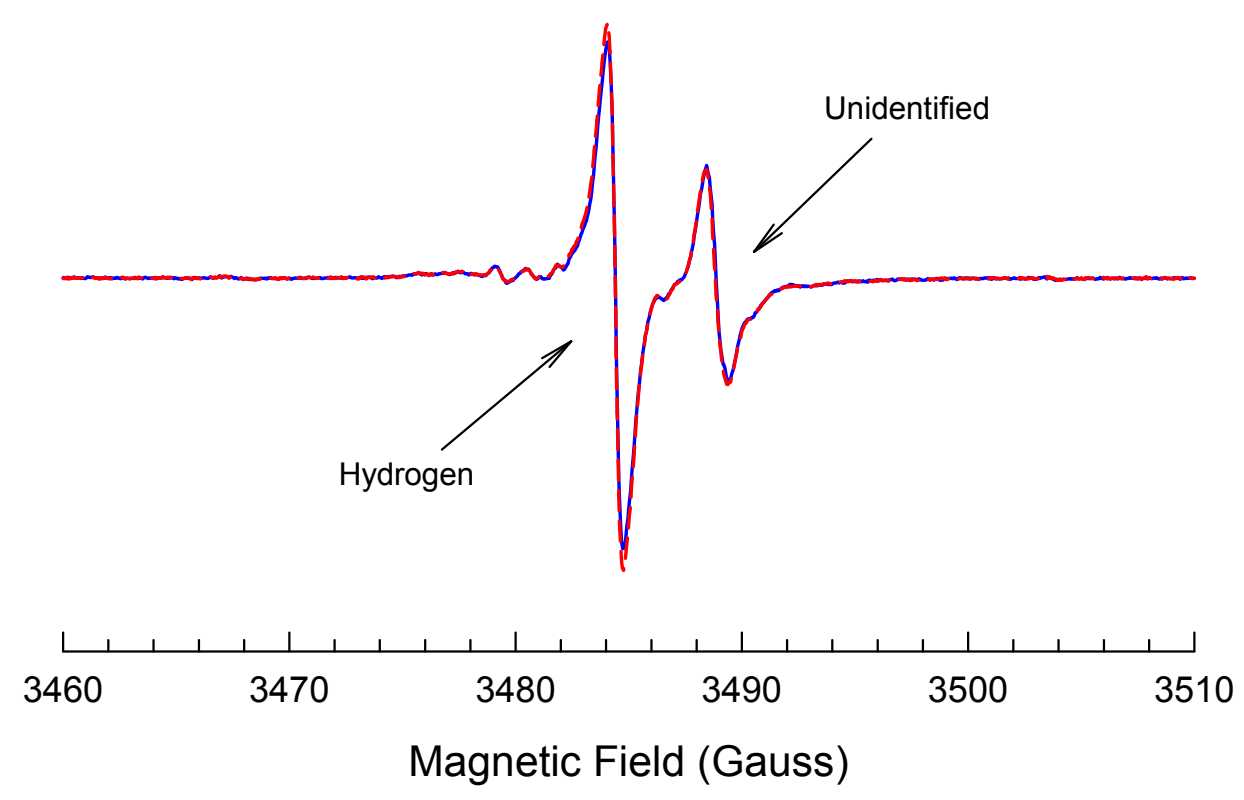

Figure 7.3. The EPR spectrum of the neutral hydrogen donor in the $700{ }^{\circ} \mathrm{C}$ annealed CrysTec sample. These data were taken at $4 \mathrm{~K}$ with the magnetic field parallel to the [001] direction. The solid blue trace was taken without any illumination, while the dashed red trace was taken during illumination with 442 $\mathrm{nm}$ laser light. The two spectra are very similar. 
of annealing in $\mathrm{N}_{2}$ gas at $700{ }^{\circ} \mathrm{C}$. The dominant EPR signal near $3484 \mathrm{G}$ has a g value along the [001] direction of $\mathrm{g}_{[001]}=1.9408$, indicating that it is the neutral hydrogen donor. This signal was present without $442 \mathrm{~nm}$ illumination. Turning on the $442 \mathrm{~nm}$ laser light at $4 \mathrm{~K}$ increased the intensity of this signal by only a small amount (less than $5 \%)$.

Figure 7.4 shows the EPR spectra taken from the $650{ }^{\circ} \mathrm{C}$ annealed MTI sample. These spectra were taken at $4 \mathrm{~K}$ with the magnetic field along the [001] direction in the crystal. The dominant EPR signal is the neutral hydrogen donor, while the doublet EPR signal on the lower field side of the hydrogen donor is the neutral fluorine donor which has been fully characterized in Chapter 6. The solid blue trace in Fig. 7.4 shows the EPR spectrum taken before any illumination, while the red dashed trace was taken during the illumination with $442 \mathrm{~nm}$ laser light. Figure 7.4 shows that the neutral fluorine donor in

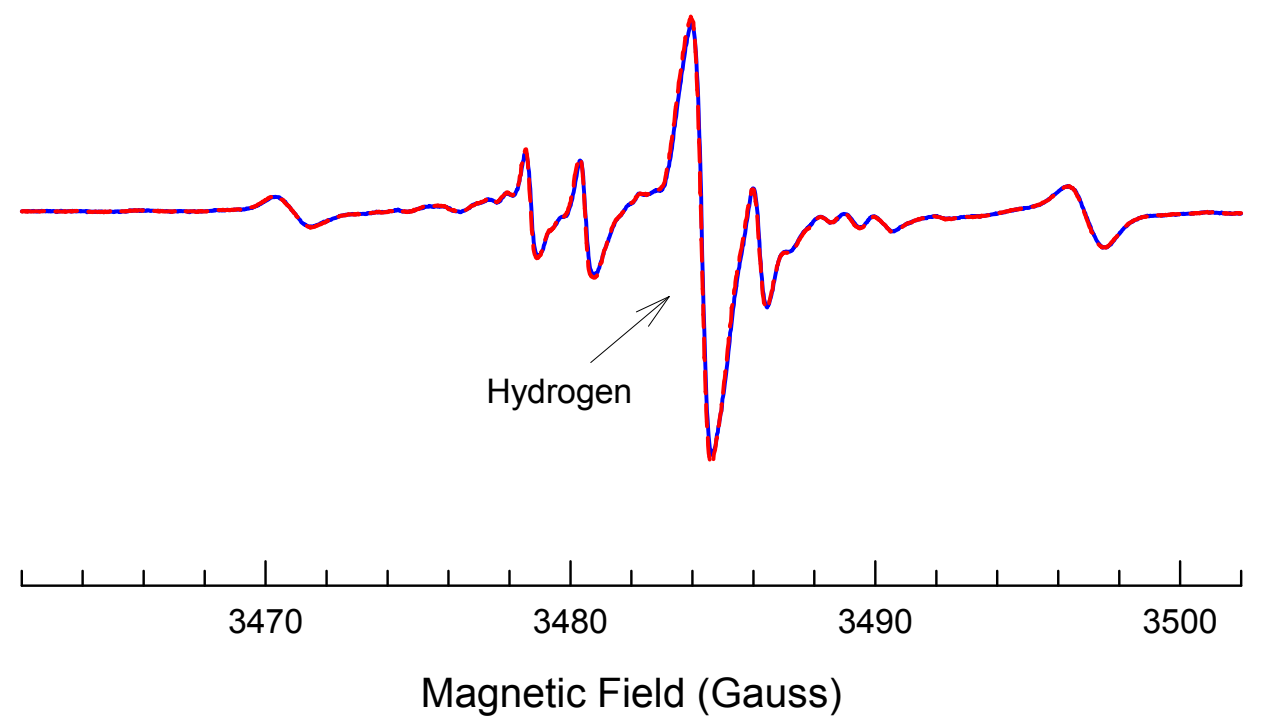

Figure 7.4. The EPR spectrum of the neutral hydrogen donor in the $650{ }^{\circ} \mathrm{C}$ annealed MTI sample. These data were taken at $4 \mathrm{~K}$ with the magnetic field along the [001] direction. The solid blue trace was taken without any illumination, while the dashed red trace was taken during illumination with $442 \mathrm{~nm}$ laser light. 
this reduced sample is not affected by the $442 \mathrm{~nm}$ laser light.

Figure 7.5 shows spectra of the neutral hydrogen donor taken from the $700{ }^{\circ} \mathrm{C}$ annealed CrysTec sample when the magnetic field is along the other two high-symmetry directions in the crystal. Trace (a) was taken with the magnetic field along the [110] direction, while trace (b) was taken with the magnetic field along the [100] direction. In Chapter 6, I showed that there are four crystallographically equivalent sites for fluorine donors. All four sites are magnetically equivalent when the magnetic field is along the [001] or [100] directions in the crystal, and the four sites fall into two sets when the magnetic field is along [110] direction. The symmetry of the neutral hydrogen donor is lower than the lattice (i.e., there are more than two crystallographically equivalent sites). My EPR data shows there are at least four crystallographically equivalent sites of the
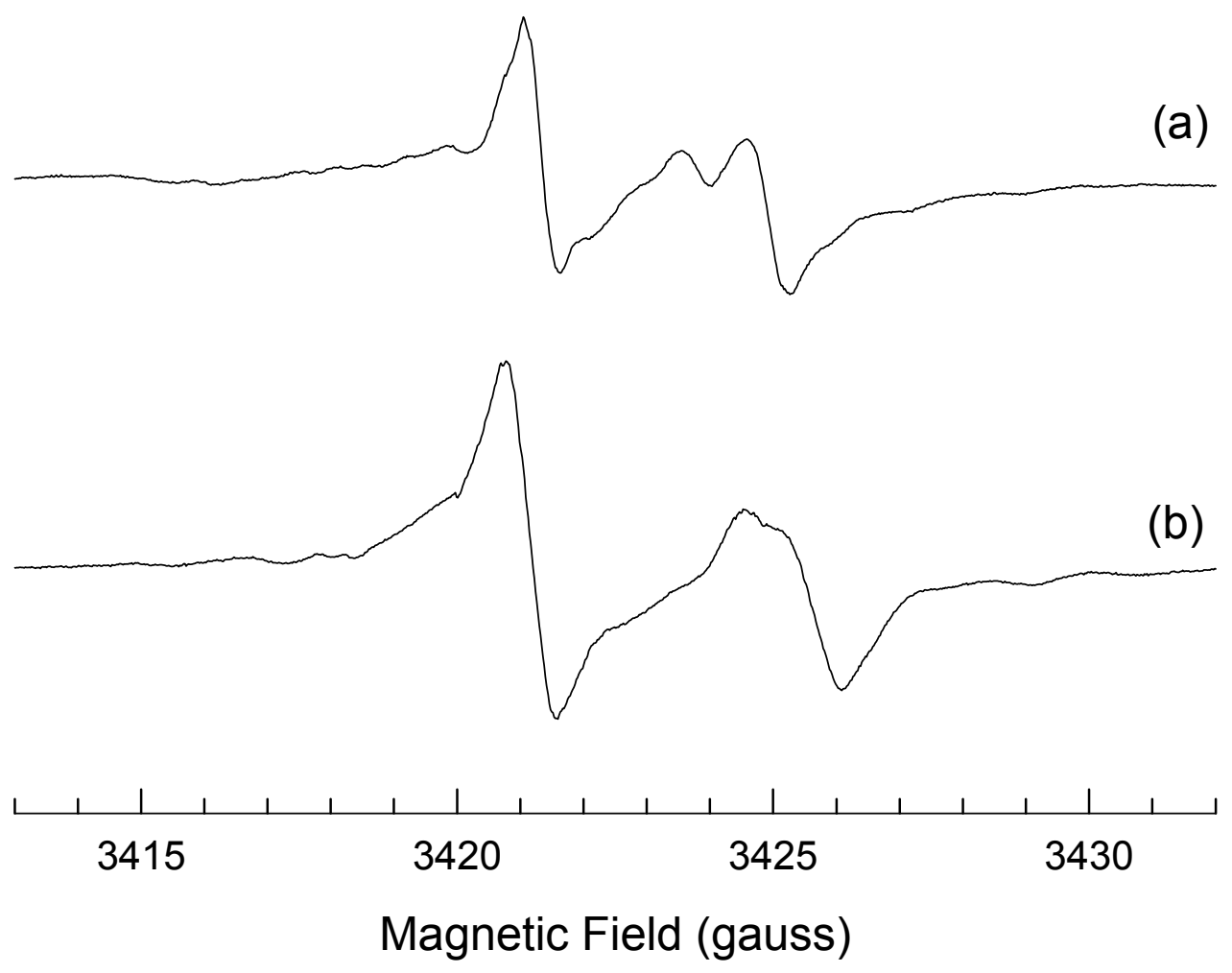

Figure 7.5. The EPR spectra of the neutral hydrogen donor in the $700{ }^{\circ} \mathrm{C}$ annealed CrysTec $\mathrm{TiO}_{2}$ crystal. These data were taken at $4 \mathrm{~K}$ with the magnetic field along (a) the [110] direction and (b) the [100] direction. 
hydrogen donor, with all four of the sites being magnetically equivalent when the magnetic field is along the [001] direction. However, these four sites fall into two sets when the magnetic field is along either the [110] or the [100] direction in the crystal, indicating that the neutral hydrogen donor has a lower symmetry than the neutral fluorine donor. The two dominant EPR signals in Fig. 7.5 are due to neutral hydrogen donors. The smaller EPR signal partly overlapping the hydrogen donor signal at higher field position in Fig. 7.5 is not identified. The [100] and [110] spectra taken from the annealed MTI sample $\left(650^{\circ} \mathrm{C}\right)$ do not show well-resolved hydrogen signals because they overlap with signals from the neutral fluorine donor.

\subsection{ENDOR Results}

Figure 7.6 shows an ENDOR spectrum taken from the $650{ }^{\circ} \mathrm{C}$ annealed MTI sample. The two dominant lines are due to the hyperfine interaction with a hydrogen

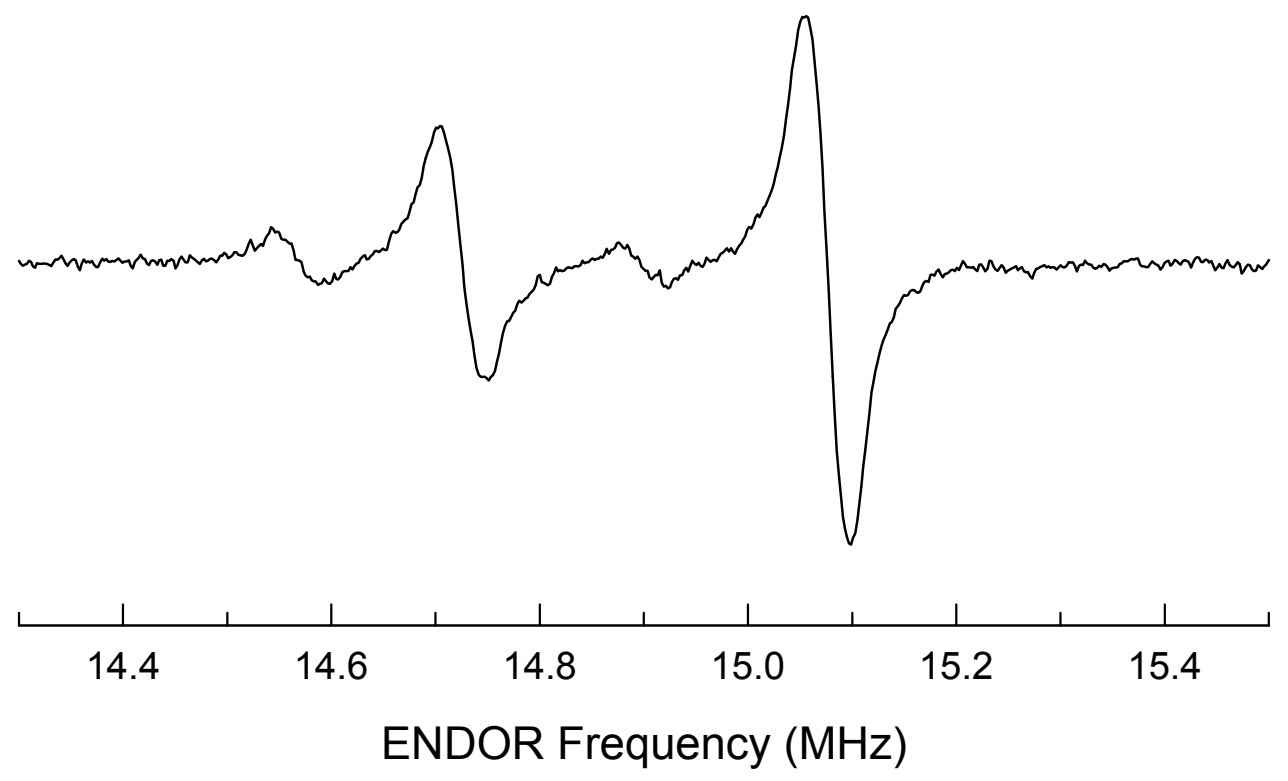

Figure 7.6. ENDOR spectrum of the neutral hydrogen donor in the $650{ }^{\circ} \mathrm{C}$ annealed $\mathrm{MTI} \mathrm{TiO}_{2}$ crystal. These data were taken at $4 \mathrm{~K}$ with the magnetic field parallel to the [001] direction. 

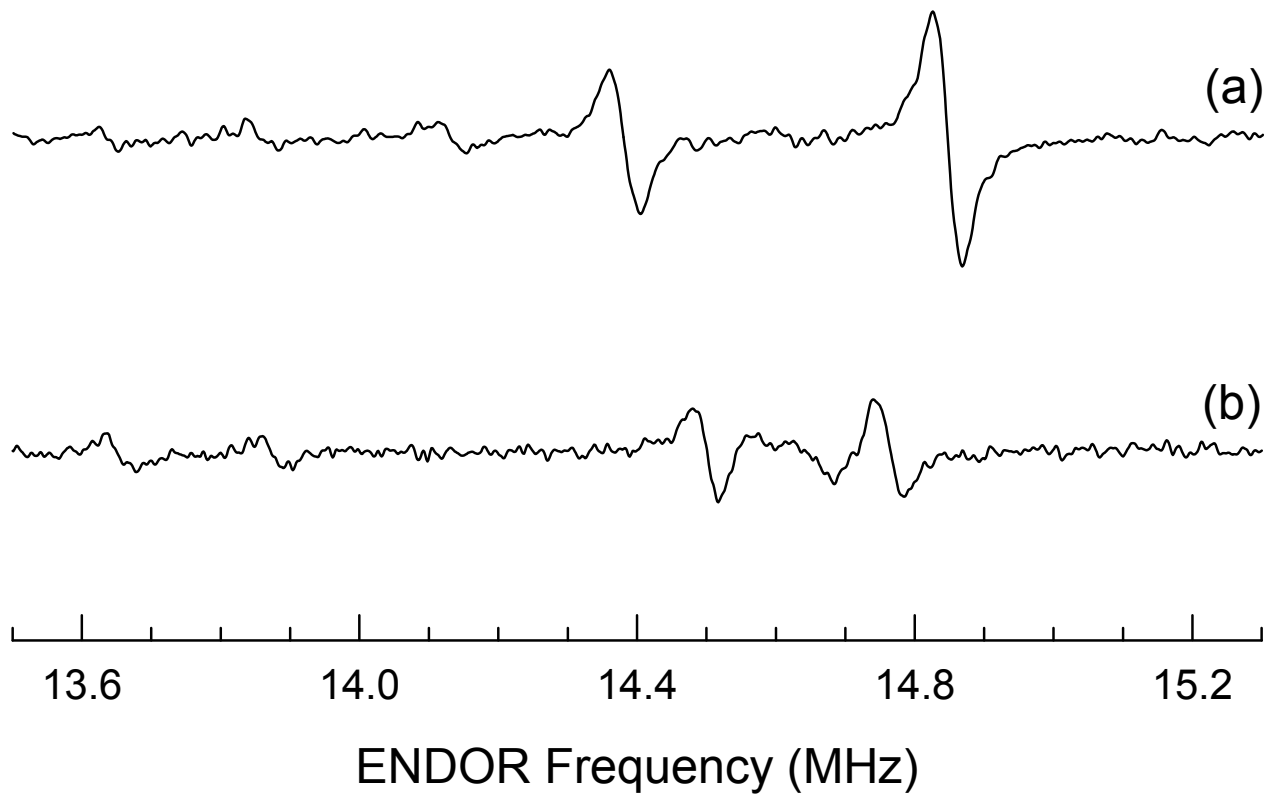

Figure 7.7. ENDOR spectrum of the neutral hydrogen donor in the $650{ }^{\circ} \mathrm{C}$ annealed $\mathrm{MTI} \mathrm{TiO}_{2}$ crystal. These data were taken at $4 \mathrm{~K}$ with the magnetic field parallel to the [110] direction.
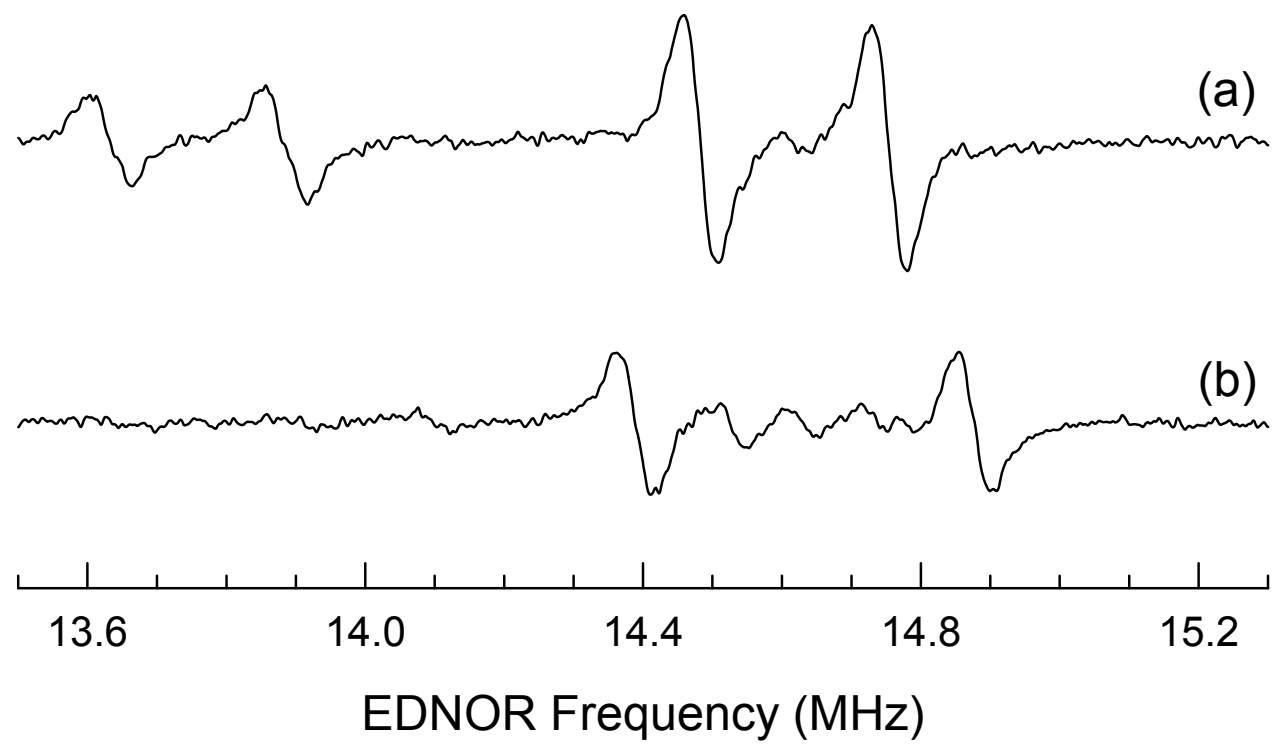

Figure 7.8. ENDOR spectrum of the neutral hydrogen donor in the $650{ }^{\circ} \mathrm{C}$ annealed $\mathrm{MTI} \mathrm{TiO}_{2}$ crystal. These data were taken at $4 \mathrm{~K}$ with the magnetic field parallel to the [100] direction. 
nucleus. The hyperfine constant is $0.35 \mathrm{MHz}$ for this direction. The small signal which resides at the center of the two dominant lines is the free spin signal of hydrogen nuclei. There is a very weak signal at $15.2 \mathrm{MHz}$, which pairs with the small signal at $14.6 \mathrm{MHz}$. These two small signals are also caused by a hyperfine interaction with hydrogen nuclei.

Figures 7.7 and 7.8 show the ENDOR spectra taken from the $650{ }^{\circ} \mathrm{C}$ annealed sample when the magnetic field is along the [110] and the [100] directions in the crystal, respectively. In both figures, the upper trace (a) was taken when the magnetic field was set on the EPR line at lower field, and the lower trace (b) was taken when the magnetic field was set on the EPR line at higher field. For example, trace (a) in Fig. 7.7 was taken with the magnetic field set at $3421 \mathrm{G}$ (see Fig. 7.5(a)), while trace (b) in Fig. 7.7 was taken with the magnetic field set on $3425 \mathrm{G}$ (see Fig. 7.5(a)).

\subsection{Discussion}

Hydrogen exists in $\mathrm{TiO}_{2}$ as interstitials and thus forms shallow donors. These shallow hydrogen donors behave very similar to the shallow fluorine donors discussed in Chapter 6 . In fully oxidized $\mathrm{TiO}_{2}$ crystals, all the hydrogen shallow donors are ionized, and thus are nonparamagnetic. The ionized hydrogen atom, which is a proton, will bond to an oxygen ion and thus form an $\mathrm{OH}^{-}$ion. At very low temperature, illumination with $442 \mathrm{~nm}$ laser light produces electron and hole pairs. The electrons can be trapped by $\mathrm{OH}^{-}$ions, where they form neutral hydrogen donors. However, instead of localizing on the proton, the trapped electron will localize on the nearest-neighbor $\mathrm{Ti}^{4+}$ ions and form $\mathrm{Ti}^{3+}$ ions. Annealing the sample at high temperature in an oxygen deficient atmosphere produces oxygen vacancies, and thus creates large concentrations of free electrons which make the sample more $n$ type. Those electrons can "freeze out" on singly ionized hydrogen donors when the sample is cooled to very low temperature and form neutral hydrogen donors. 
The site splitting pattern of the EPR spectra with the magnetic field along [100] and [110] indicate that the hydrogen donor has low symmetry. The EPR spectra along the three high-symmetry orientations show that the g matrix is nearly axial, and the unique axis is along the [001] direction of the crystal. This behavior is very similar to the fluorine donor. In addition, the $\mathrm{g}$ values of the hydrogen donor are very similar to those of the fluorine donor. The EPR lines of fluorine and of hydrogen are always next to each other when the magnetic field is along an arbitrary direction in the crystal. Together, these observations strongly suggest that the electron trapped by hydrogen is localized on an adjacent $\mathrm{Ti}^{3+}$ ion like in the case of the fluorine donor. A small hyperfine constant $(0.5 \mathrm{MHz})$ indicates the trapped electron interacts weakly with the hydrogen nucleus.

Both the hydrogen and the fluorine are shallow donors in $\mathrm{TiO}_{2}$ crystals. In particular, the hydrogen donor is closer to the conduction band than the fluorine donor. In oxidized crystals containing both hydrogen and fluorine impurities, only the neutral fluorine donor can be observed with $442 \mathrm{~nm}$ laser light illumination at low temperature (4 K). A slight reduction (e.g., 60 minutes at $600{ }^{\circ} \mathrm{C}$ ) drives oxygen out of the crystal and creates free electrons, which can "freeze out" at fluorine sites. Thus, $442 \mathrm{~nm}$ laser light is not needed to produce the neutral fluorine donor. Further reducing (e.g., 60 minutes at $600{ }^{\circ} \mathrm{C}+50$ minutes at $650{ }^{\circ} \mathrm{C}$ ) ensures more electrons are available for shallow donors when more oxygen vacancies are produced. A portion of the free electrons will be trapped by ionized hydrogen donors after all the fluorine donors are converted to the neutral charge state. The neutral hydrogen donor signals can then be observed. In the samples containing no fluorine impurities (e.g., in the CrysTec samples), neutral hydrogen donor signals can be easily observed with $442 \mathrm{~nm}$ laser light illumination at very low temperature $(4 \mathrm{~K})$.

In order to obtain a complete set of spin-Hamiltonian parameters (both the $\mathrm{g}$ matrix and the hyperfine matrix), angular dependence studies of the EPR and ENDOR spectra are necessary. Experimentally, I found that it was very difficult to produce an 
optimum sample for these studies. In other words, it was not easy to anneal the sample the "right amount" since the hydrogen signal intensity did not behave linearly with annealing temperature and time. An excess anneal (either too high a temperature or too long a time) and an insufficient anneal will both result in weak EPR and ENDOR signals. In over 12 annealed samples, the $650{ }^{\circ} \mathrm{C}$ annealed MTI sample showed the largest signals. The ENDOR spectrum due to hydrogen can be observed when the magnetic field is along any direction. However, the EPR signals of fluorine are not resolved from those of hydrogen for many directions of magnetic field, thus the angular dependence study of hydrogen donor is not as promising. The CrysTec samples are better candidates for angular dependence studies of the hydrogen donor because they do not contain fluorine donors. Systematic annealing could help select CrysTec samples for use in future studies of the hydrogen donor in $\mathrm{TiO}_{2}$. 


\section{Chapter 8}

\section{Sodium Interstitials in $\mathrm{TiO}_{2}$ Crystals}

\subsection{EPR Results}

Several very weak EPR signals exhibiting four-line patterns were observed in single crystals of $\mathrm{TiO}_{2}$ during my earlier studies of oxygen vacancies. These four-line EPR spectra can be easily observed in the as-received MTI samples, where their intensities are comparable to the neutral oxygen vacancies. Figure 8.1 shows a spectrum of these fourline EPR signals. These data were taken from as-received $\mathrm{MTI} \mathrm{TiO}_{2}$ crystal at $30 \mathrm{~K}$ with the magnetic field along the [001] direction in the crystal. Similar to oxygen vacancies, illumination with laser light is necessary in order to produce these four-line signals. A

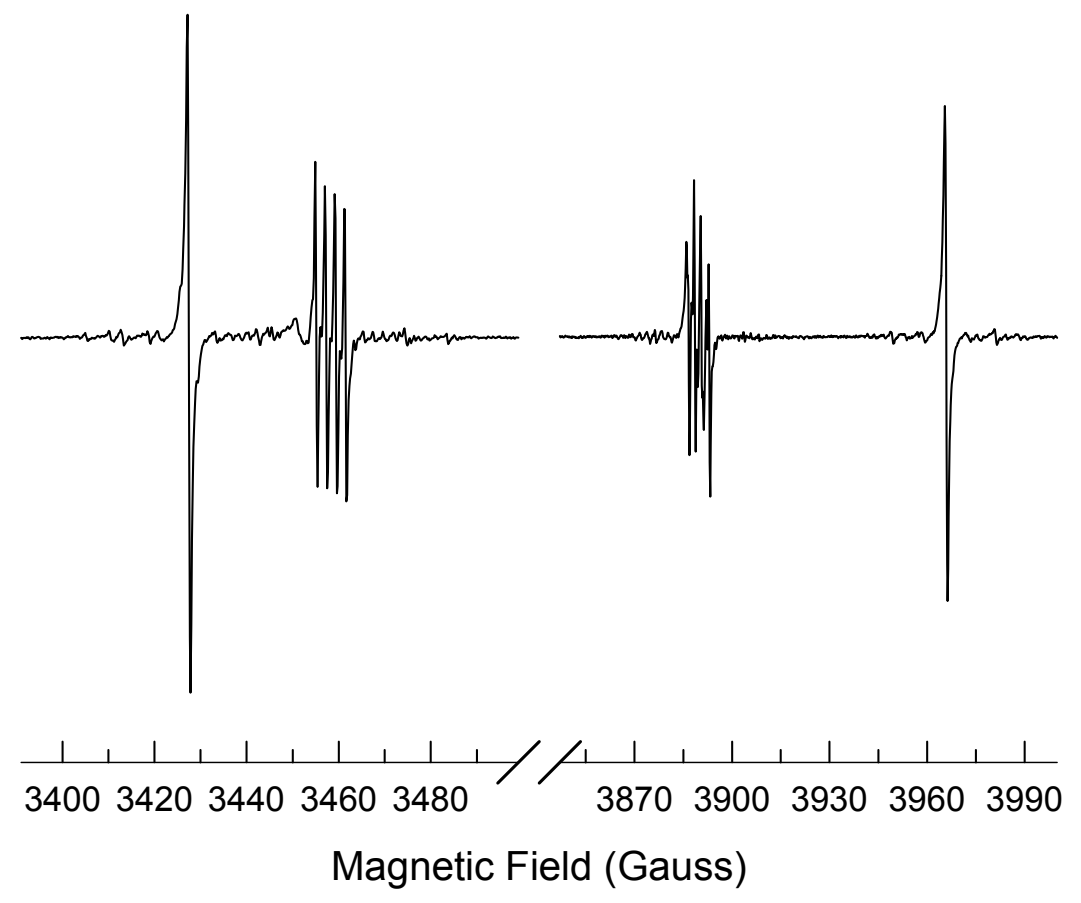

Figure 8.1. The four-line EPR spectrum taken from an as-received $\mathrm{MTI} \mathrm{TiO}_{2}$ crystals These data were taken at $30 \mathrm{~K}$ with the magnetic field along the [001] direction in the crystal. 
$442 \mathrm{~nm} \mathrm{He}-\mathrm{Cd}$ laser was used in this study. The two sharp single lines at the two ends of the spectrum are neutral oxygen vacancy EPR signals, while the two sets of four-line EPR signals are the same as those observed in the Namiki sample (shown in Chapter 4). The two sets of four-line EPR signals belong to a spin $\mathrm{S}=1$ system. This $\mathrm{S}=1$ electron
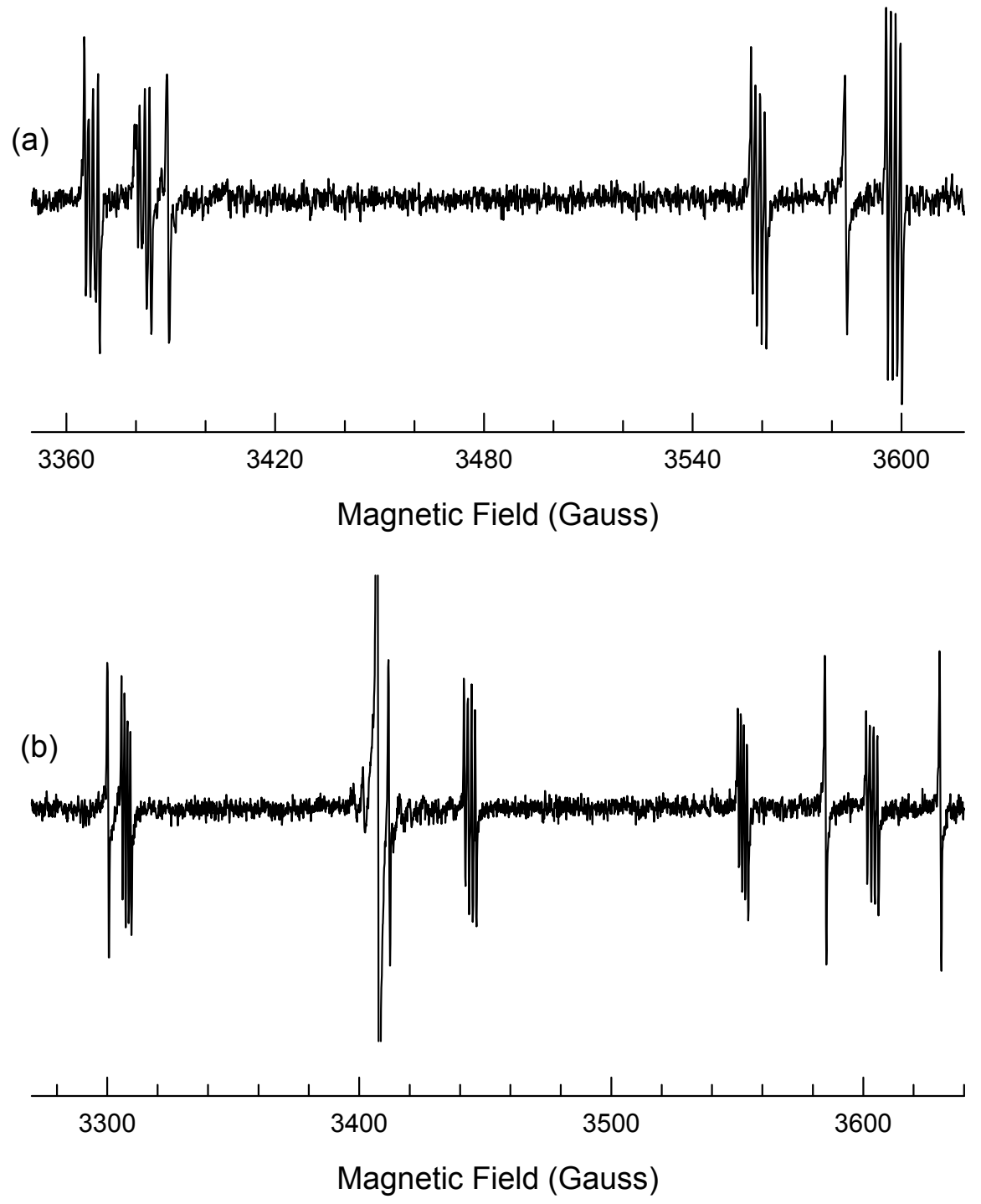

Figure 8.2. The four-line EPR spectrum taken from an as-received $\mathrm{MTI} \mathrm{TiO}_{2}$ crystals. These data were taken at $30 \mathrm{~K}$ with the magnetic field along (a) the [110] direction and (b) the [100] direction in the crystal. 
system interacts with a nucleus having $\mathrm{I}=3 / 2$ and nearly $100 \%$ abundance. The most likely candidates are sodium $\left({ }^{23} \mathrm{Na}\right)$ and lithium $\left({ }^{7} \mathrm{Li}\right)$ nuclei.

Figure 8.2 shows the EPR spectra of the four-line signals taken with the magnetic field along the [110] direction and the [100] direction in the crystal. The four sharp single-line signals in each trace are due to neutral oxygen vacancies. The fifth sharp single-line signal in trace (b) is the signal from singly ionized oxygen vacancies. In both traces, there are four sets of four-line signals instead of two sets as shown in Fig. 8.1. Similar to the neutral oxygen vacancy center discussed in Chapter 4, there are a total of four crystallographically equivalent defect sites for the four-line signals. The splitting from two sets of four-line signals into four sets is due to magnetically inequivalent sites. When the magnetic field is along the [001] direction in the crystal, all four sites are magnetically equivalent, therefore, only two set of four-line signals are observed. The four sites fall into two groups when the magnetic field rotates away from the [001] to the [110] direction or from the [001] to the [100] direction. The two sites in each group are magnetically equivalent, and thus two sets of lines for $\mathrm{S}=1$ are observed. When the magnetic field is along an arbitrary direction with lower symmetry, all four sites are not magnetically equivalent and thus a total of eight sets of four-line signals will be observed.

\subsection{Annealing Experiment}

The four-line signals disappear when the sample is annealed at high temperature (e.g., $600{ }^{\circ} \mathrm{C}$ ) in $\mathrm{N}_{2}$ gas. After annealing, illumination at $30 \mathrm{~K}$ does not regenerate the signals. One interpretation of these results requires the responsible defects to be mobile and easily diffuse out of the crystal. The hyperfine structure suggests that sodium (Na) and lithium (Li) are most likely candidates. A series of annealing studies were carried out to determine whether lithium or sodium is the active impurity. Three sodium and lithium compounds were used in these anneals, $\mathrm{LiF}$ (powder), $\mathrm{NaOH}$ (flakes), and $\mathrm{LiOH}$ (flakes). 
For the $\mathrm{LiF}$ anneal, the sample was buried under the $\mathrm{LiF}$ powder in an alumina boat, and then heated for 30 minutes at $700{ }^{\circ} \mathrm{C}$. The EPR results from the $\mathrm{LiF}$ annealed sample are similar to the result from the $\mathrm{N}_{2}$ gas annealed sample. All of the four-line EPR signals disappeared and the sample became very conductive.

For the $\mathrm{NaOH}$ anneal, the $\mathrm{TiO}_{2}$ sample was buried under a fine powder of $\mathrm{NaOH}$ (the flakes were ground into a fine powder) and then heated for 60 minutes at $450{ }^{\circ} \mathrm{C}$. More than $50 \%$ percent of the sample was destroyed (i.e., disappeared). The reaction is as following.

$$
\mathrm{TiO}_{2}+2 \mathrm{NaOH}=\mathrm{NaTiO}_{3}+\mathrm{H}_{2} \mathrm{O}
$$

Figure 8.3 shows the EPR spectra of the four-line signals taken from the $\mathrm{TiO}_{2}$
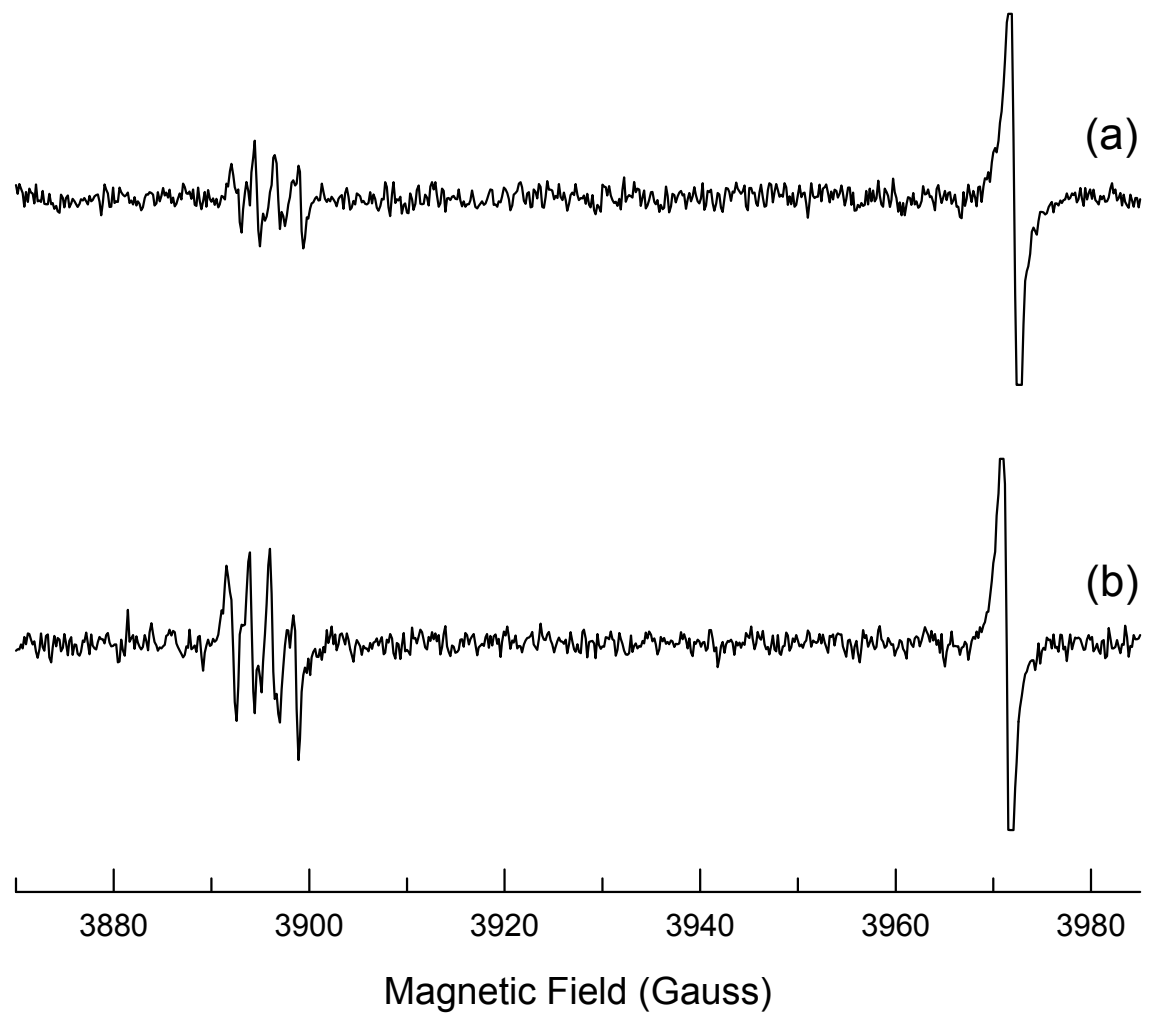

Figure 8.3. The four-line pattern EPR spectra taken from (a) the as-received MTI sample and (b) the $\mathrm{NaOH}$ annealed MTI sample. These data were taken at $30 \mathrm{~K}$ with the magnetic field along the [001] direction. 
samples before and after $\mathrm{NaOH}$ annealing. Trace (a) was taken from the as-received sample and trace (b) was taken after the anneal in $\mathrm{NaOH}$. These two traces were taken under the same conditions, i.e., at $30 \mathrm{~K}$ with the magnetic field along the [001] direction and during illumination with $442 \mathrm{~nm}$ laser light. For a clearer view, only the set of fourline signals at high field (near $3895 \mathrm{G}$ ) is shown. The single line signal near $3970 \mathrm{G}$ is due to neutral oxygen vacancies.

The annealing treatment using $\mathrm{LiOH}$ is similar to that of $\mathrm{NaOH}$. The sample was
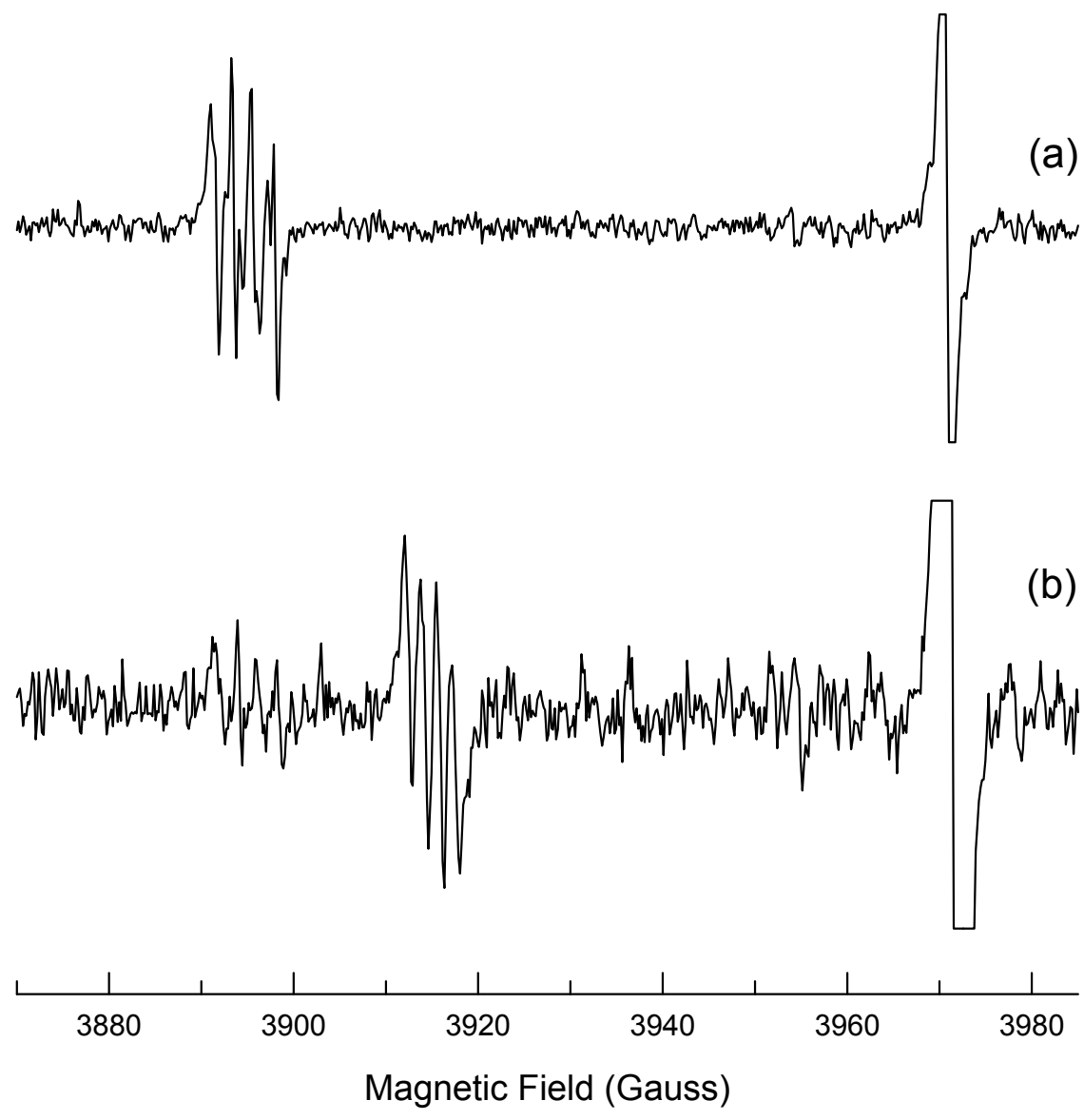

Figure 8.4. The four-line pattern EPR spectra taken from (a) the as-received MTI sample and (b) the $\mathrm{LiOH}$ annealed MTI sample. These data were taken at $30 \mathrm{~K}$ with the magnetic field along the [001] direction. 
heated at $450{ }^{\circ} \mathrm{C}$ for 60 minutes. The $\mathrm{LiOH}$ did not corrode the $\mathrm{TiO}_{2}$ sample. Figure 8.4 shows EPR spectra of the four-line pattern signals taken from the $\mathrm{TiO}_{2}$ sample before and after LiOH annealing. Trace (a) was taken from the as-received sample. Then the sample was annealed in $\mathrm{LiOH}$ and trace (b) was taken from the annealed sample. The conditions of the system were kept the same when taking traces (a) and (b). Comparing the two traces, the set of four-line signals near $3895 \mathrm{G}$ is decreased after annealing, and a new set of four-line signals appeared near 3915 G. Another new set of four-line signal appeared at the lower field value of $3485 \mathrm{G}$. These two new sets of four-line signals form an $\mathrm{S}=1$ system, which is very similar to the old $\mathrm{S}=1$ system shown in Fig. 8.1.

The production of a new $\mathrm{S}=1$ system and decrease of the previous $\mathrm{S}=1$ system strongly suggest that the four-line EPR signals in as received samples are a result of sodium $(\mathrm{Na})$ interstitials. Annealing the Na-containing $\mathrm{TiO}_{2}$ in $\mathrm{LiOH}$ diffuses $\mathrm{Na}$ ions out of the crystal and diffuses Li ions into the crystal. The Li ions that diffused into the crystal forms new interstitial defects and act as donors.

\subsection{Spin Hamiltonian}

Figure 8.5 shows the EPR angular dependence of the Na center. Comparing the angular dependence data of the $\mathrm{Na}$ center with that of neutral oxygen vacancies, one can conclude that these two centers have similar origins. I propose that the model for the $\mathrm{Na}$ center is an interstitial $\mathrm{Na}^{+}$ion next to a neutral oxygen vacancy. The effective charge state of a doubly ionized oxygen vacancy $\left(\mathrm{V}_{\mathrm{o}}^{++}\right)$is +2 , which will repel the +1 charged $\mathrm{Na}^{+}$ion. Thus, the $\mathrm{Na}^{+}$will stay away from doubly ionized oxygen vacancies. I suggest the interstitial $\mathrm{Na}^{+}$ion is mobile in $\mathrm{TiO}_{2}$ crystals. When two electrons are trapped on $\mathrm{V}_{\mathrm{o}}^{++}$and change it to $\mathrm{V}_{\mathrm{o}}{ }^{0}$, the local charge state becomes zero. Then the mobile $\mathrm{Na}^{+}$ion can be 'trapped' near the oxygen vacancy.

I also observed a four-line signal at magnetic field values near the singly ionized oxygen vacancies. This four-line signal is very unstable, it can only be observed for a 
very short moment when $442 \mathrm{~nm}$ laser light was removed and the Na center with $\mathrm{S}=1$ starts to decay away. The phenomenon indicates that once the trapped electron was thermally released, the $\mathrm{Na}^{+}$ion is repelled by the effectively positively charged oxygen vacancy.

The g matrix and the D matrix of the $\mathrm{Na}$ interstitial center is listed in Table 8.1. These matrices are generated by analyzing the experimental data using a least-squares fitting program. I note that the parameters of the $g$ matrix and $\mathrm{D}$ matrix are very similar to the matrices describing the neutral oxygen vacancy.

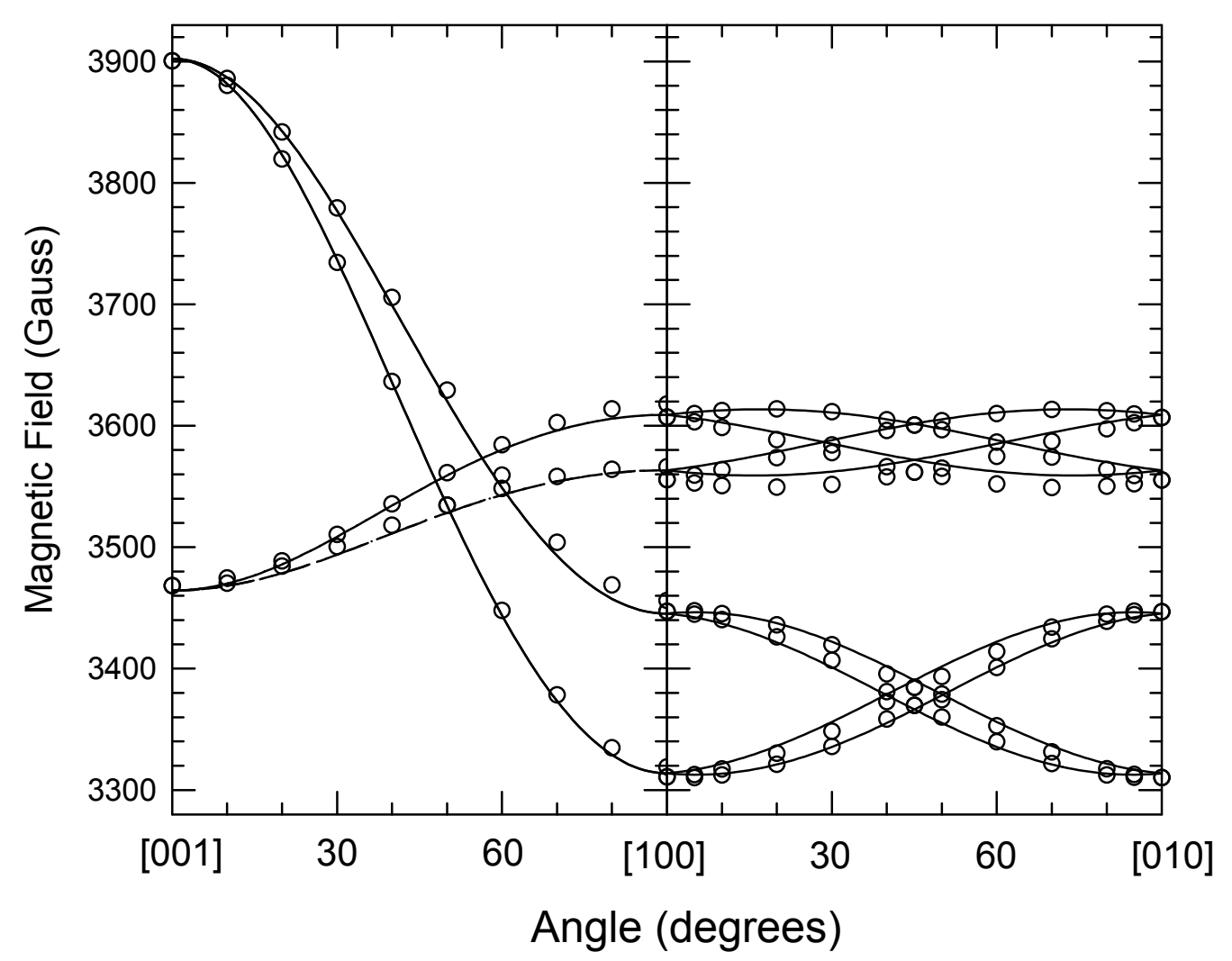

Figure 8.5. Angular dependence of the g matrix and $\mathrm{D}$ matrix for the $\mathrm{Na}^{+}-\mathrm{V}_{\mathrm{o}}{ }^{0}$ center in $\mathrm{TiO}_{2}$. These EPR data were acquired in the two high-symmetry planes of the crystal. The open circles are experimental data, and the solid curves are generated using the best-fit $g$ and $\mathrm{D}$ matrices. 
Table 8.1. Spin-Hamiltonian parameters of the $\mathrm{Na}^{+}-\mathrm{V}_{\mathrm{O}}^{0}$ center. These parameters are generated by analyzing the EPR angular dependence data using a least-squares fitting MATLAB program.

\begin{tabular}{|c|c|c|c|c|}
\hline \multirow{2}{*}{ Center } & \multicolumn{2}{|c|}{ Principal Values } & \multicolumn{2}{c|}{ Principal Axes } \\
\cline { 3 - 5 } & \multicolumn{2}{|c|}{} & $\theta(\mathrm{deg})$ & $\phi(\mathrm{deg})$ \\
\hline \multirow{3}{*}{$\mathrm{Na}^{+}-\mathrm{V}_{\mathrm{O}}^{0}$} & $\mathrm{~g}_{1}$ & 1.9551 & 90.0 & 1.7 \\
\cline { 2 - 5 } & $\mathrm{g}_{2}$ & 1.9306 & 90.0 & 91.7 \\
\cline { 2 - 5 } & $\mathrm{g}_{3}$ & 1.8378 & 0.0 & 0.0 \\
\cline { 2 - 5 } & $\mathrm{D}_{1}$ & 272.80 & 90 & 81.7 \\
\cline { 2 - 5 } & $\mathrm{D}_{2}$ & 102.70 & 90 & 171.7 \\
\cline { 2 - 5 } & $\mathrm{D}_{3}$ & -375.50 & 0.0 & 0.0 \\
\hline
\end{tabular}




\section{References}

1.1. F. A. Grant, Rev. Mod. Phys. 31, 646 (1959).

1.2. A. Fujishima, X. Zhang, and D. A. Tryk, Surf. Sci. Rep. 63, 515 (2008).

1.3. C. L. Pang, R. Lindsay, and G. Thornton, Chem. Soc. Rev. 37, 2328 (2008).

1.4. K. Shankar, J. I. Basham, N. K. Allam, O. K. Varghese, G. K. Mor, X. Feng, M. Paulose, J. A. Seabold, K. S. Choi, and C. A. Grimes, J. Phys. Chem. C 113, 6327 (2009).

1.5. V. N. Kuznetsov and N. Serpone, J. Phys. Chem. B 110, 25203 (2006).

1.6. C. Di Valentin, E. Finazzi, G. Pacchioni, A. Selloni, S. Livraghi, M. C. Paganini, and E. Giamello, Chem. Phys. 339, 44 (2007).

1.7. M. K. Nowotny, L. R. Sheppard, T. Bak, and J. Nowotny, J. Phys. Chem. C 112, 5275 (2008).

1.8. A. Fujishima and K. Honda, Nature 238, 37 (1972).

1.9. G. K. Mor, M. A. Garvalho, O. K. Varghese, M. V. Pishko, C. A. Grimes, J. Mater. Res. 19, 628 (2004)

1.10. R. Asahi, T. Morikawa, T. Ohwaki, K. Aoki, and Y. Taga, Science, 293, 269 (2001)

1.11. J. -M. Spaeth and H. Overhof, "Point Defects in Semiconductors and Insulators: Determination of Atomic and Electronic Structure from Paramagnetic Hyperfine Interactions," Springer Series of Materials Science, Vol. 51 (Springer Verlag, Heidelberg, 2003).

1.12. F. C. Verduin, US 2290922 patent (1942).

1.13. T. K. Pong, John Besida, T. A. O'Donnell, D. G. Wood, Ind. Eng. Chem. Res. 34, 308 (1995).

1.14. S. Zerfoss, R. G. Stokes, and C. H. Moore, Jr., J. Chem. Phys. 16, 1166 (1946).

1.15. C. Kooij and H. J. M. Couwenberg, Philips Technical Review 23, 161 (1962).

1.16. M. Higuchi, T. Hosokawa, and S. Kimura, J. Cryst. Growth 112, 354 (1991). 
1.17. M. E. Straumanis, T. Ejima, and W. J. James, Acta Cryst. 14, 493 (1961)

1.18. R. A. Parker, Phys. Rev. 124, 1719 (1961).

2.1. S. D. Setzler, K. T. Stevens, N. C. Fernelius, M. P. Scripsick, G. J. Edwards, and L. E. Halliburton, J. Phys.: Condens. Matter 15, 3969 (2003).

2.2. Shan Yang and L. E. Halliburton, Phys. Rev. B 81, 035204 (2010).

2.3. K. T. Stevens, Ph.D. Dissertation, West Virginia University (1999).

2.4. J. E. Wertz and J. R. Bolton, “Electron Spin Resonance Elementary Theory and Practical Applications," (McGraw-Hill Series in Advanced Chemistry, 1972).

2.5. S. D. Setzler, Ph.D. Dissertation, West Virginia University (1998).

2.6. J. -M. Spaeth, J. R. Niklas, and R. H. Bartram, "Structural Analysis of Point Defects in Solids" (Springer-Verlag, Berlin, 1992).

3.1. H. J. Gerritsen, S. E. Harrison, H. R. Lewis, and J. P. Wittke, Phys. Rev. Lett. 2, 153 (1959).

3.2. B. W. Faughnan and Z. J. Kiss, Phys. Rev. Lett. 21, 1331 (1968).

3.3. H. J. Gerritsen, and E. S. Sabisky, Phys. Rev. 125, 1853 (1962).

3.4. T. C. Ensign, Te-Tse Chang, and A. H. Kahn, Phys. Rev. 188, 703 (1969).

3.5. H. J. Gerritsen and H. R. Lewis, Phys. Rev. 119, 1010 (1960).

3.6. P. F. Chester, J. Appl. Phys. 32, 866 (1961).

3.7. P. H. Zimmermann, Phys. Rev. B 8, 3917, (1973).

3.8. Te-Tse Chang, Phys. Rev. 136, A1413 (1964).

3.9. Te-Tse Chang, Phys. Rev. 147, 264 (1966).

3.10. K. W. Blazey, K. A. Muller, and W. Berlinger, Solid State Commun. 54, 1039 (1985).

3.11. H. G. Andersen, Phys. Rev. 120, 1606 (1960).

3.12. S. Guler, B. Rameev, R. I. Khaibullin, O. N. Lopatin, and B. Aktas, J. Phys.: Conf. Ser. 153, 012052 (2009).

3.13. D. Zwingel, Solid State Commun. 20, 397 (1976).

3.14. D. Zwingel, Solid State Commun. 26, 775 (1978). 
3.15. H. J. Gerritsen and E. S. Sabisky, Phys. Rev. 125, 1853 (1962).

3.16. R. B. Bossoli, T. J. Welsh, and O. R. Gilliam, Phys. Rev. B 19, 4376 (1976).

3.17. P. I. Kingsbury, Jr., W. D. Ohlsen, and O. W. Johnson, Phys. Rev. 175, 1091 (1968).

3.18. P. F. Chester, J. Appl. Phys. 32, 2233 (1961).

3.19. J. Kerssen and J. Volger, Physica 69, 535 (1973).

4.1. P. F. Chester, J. Appl. Phys. 32, 2233 (1961).

4.2. P. I. Kingsbury, W. D. Ohlsen, and O. W. Johnson, Phys. Rev. 175, 1091 (1968).

4.3. J. Kerssen and J. Volger, Physica 69, 535 (1973).

4.4. M. Aono and R. R. Hasiguti, Phys. Rev. B 48, 12406 (1993).

4.5. H. J. Gerritsen, S. E. Harrison, H. R. Lewis, and J. P. Wittke, Phys. Rev. Lett. 2, 153 (1959).

4.6. D. L. Carter and A. Okaya, Phys. Rev. 118, 1485 (1960).

4.7. G. J. Lichtenberger and J. R. Addison, Phys. Rev. 184, 381 (1969).

4.8. S. S. Kim, S. S. Jun, and M. J. Park, J. Korean Phys. Soc. 23, 73 (1990).

4.9. B. W. Faughnan and Z. J. Kiss, Phys. Rev. Lett. 21, 1331 (1968).

4.10. T. Purcell and R. A. Weeks, J. Chem. Phys. 54, 2800 (1971).

4.11. D. Zwingel, Solid State Commun. 20, 397 (1976).

5.1. K. T. Stevens, N. Y. Garces, L. E. Halliburton, M. Yan, N. P. Zaitseva, J. J. DeYoreo, G. C. Catella, and J. R. Luken, Appl. Phys. Lett. 75, 1503 (1999).

6.1. K. Shankar, J. I. Basham, N. K. Allam, O. K. Varghese, G. K. Mor, X. Feng, M. Paulose, J. A. Seabold, K. S. Choi, and C. A. Grimes, J. Phys. Chem. C 113, 6327 (2009).

6.2. J. -M. Spaeth and H. Overhof, "Point Defects in Semiconductors and Insulators: Determination of Atomic and Electronic Structure from Paramagnetic Hyperfine Interactions," Springer Series of Materials Science, Vol. 51 (Springer Verlag, Heidelberg, 2003). 
6.3. Shan Yang, L. E. Halliburton, A. Manivannan, P. H. Bunton, D. B. Baker, M. Klemm, S. Horn, and A. Fujishima, Appl. Phys. Lett. 94, 162114 (2009).

6.4. H. J. Meng, D. L. Hou, L. Y. Jia, X. J. Ye, H. J. Zhou, and X. L. Li, J. Appl. Phys. 102, 073905 (2007).

6.5. Q. Zhao, P. Wu, B. L. Li, Z. M. Lu, and E. Y. Jiang, J. Appl. Phys. 104, 073911 (2008).

6.6. S. Zhou, E. Cizmar, K. Potzger, M. Krause, G. Talut, M. Helm, J. Fassbender, S. A. Zvyagin, J. Wosnitza, and H. Schmidt, Phys. Rev. B 79, 113201 (2009).

6.7. J. C. Yu, J. Yu, W. Ho, Z. Jiang, and L. Zhang, Chem. Mater. 14, 3808 (2002).

6.8. A. M. Czoska, S. Livraghi, M. Chiesa, E. Giamello, S. Agnoli, G. Granozzi, E. Finazzi, C. Di Valentin, and G. Pacchioni, J. Phys. Chem. C 112, 8951 (2008).

6.9. C. Di Valentin, E. Finazzi, G. Pacchioni, A. Selloni, S. Livraghi, A. M. Czoska, M. C. Paganini, and E. Giamello, Chem. Mater. 20, 3706 (2008).

6.10. Q. Wang, C. Chen, W. Ma, H. Zhu, and J. Zhao, Chem. Eur. J. 15, 4765 (2009).

6.11. H. J. Gerritsen, S. E. Harrison, H. R. Lewis, and J. P. Wittke, Phys. Rev. Lett. 2, 153 (1959).

6.12. D. L. Carter and A. Okaya, Phys. Rev. 118, 1485 (1960).

6.13. G. J. Lichtenberger and J. R. Addison, Phys. Rev. 184, 381 (1969).

6.14. Shan Yang and L. E. Halliburton, "Hydrogen Donors Studied by EPR in TiO2 Crystals" (to be published).

6.15. S. C. Abrahams and J. L. Bernstein, J. Phys. Chem. 55, 3206 (1971).

6.16. P. H. Kasai, Phys. Lett. 7, 5 (1963).

6.17. J. E. Wertz and J. R. Bolton, "Electron Spin Resonance: Elementary Theory and Practical Applications,” (McGraw-Hill, New York, 1972) pp. 278-281.

7.1. M. Wohlecke and L. Kovacs, Critical Reviews in Solid State and Material Sciences 25, $1(2001)$.

7.2. C. G. Van de Walle, Phys. Rev. Lett. 85, 1012 (2000). 
7.3. D. M. Hofmann, A. Hofstaetter, F. Leiter, H. Zhou, F. Henecker, B. K. Meyer, S. B. Orlinskii, J. Schmidt, and P. G. Baranov, Phys. Rev. Lett. 88, 045504 (2002).

7.4. O. W. Johnson, W. D. Ohlsen, and P. I. Kingsbury, Phys. Rev. 175, 1102 (1968).

7.5. Shengqiang Zhou, E. Cizmar, K. Potzger, M. Krause, G. Talu, M. Helm, J. Fassbender, S. A. Zvyagin, J. Wosnitza, and H. Schmidt, Phys. Rev. B 79, 113201 (2009).

7.6. P. F. Chester, J. Appl. Phys. 32, 2233 (1961). 


\section{Appendix A}

\section{The g Matrix Analysis of $\mathrm{Ti}^{3+}$ Ions in $\mathrm{TiO}_{2}$}

The $g$ matrix of a spin system, to first order, has the form

$$
\mathrm{g}=g_{e} 1+2 \lambda \Lambda
$$

where $\Lambda_{i j}=-\sum_{n \neq G} \frac{\left\langle G\left|\hat{L}_{i}\right| n\right\rangle\left\langle n\left|\hat{L}_{j}\right| G\right\rangle}{E_{n}-E_{G}}$ is a tensor resulting from the crystal field (the crystal field removes the degeneracy of the ground energy, thus cause the an isotropic of the $g$ values). $\hat{L}_{i}$ and $\hat{L}_{j}$ are orbital angular momentum operators. $\mathrm{i}, \mathrm{j}=\mathrm{x}, \mathrm{y}$, or $\mathrm{z}$.

In $\mathrm{TiO}_{2}, \mathrm{Ti}^{3+}$ ions possess a $3 \mathrm{~d}^{1}$ electron. This $\mathrm{Ti}^{3+}$ ion lies in the center of a distorted octahedron with one oxygen ion at each of six apexes. For simplicity, a new coordinate system of $\mathrm{x}, \mathrm{y}, \mathrm{z}$ is chosen. The relative relation between the $\mathrm{x}, \mathrm{y}$, and $\mathrm{z}$ axes and the three high-symmetry orientations in the $\mathrm{TiO}_{2}$ crystal is illustrated in Fig. A.1.

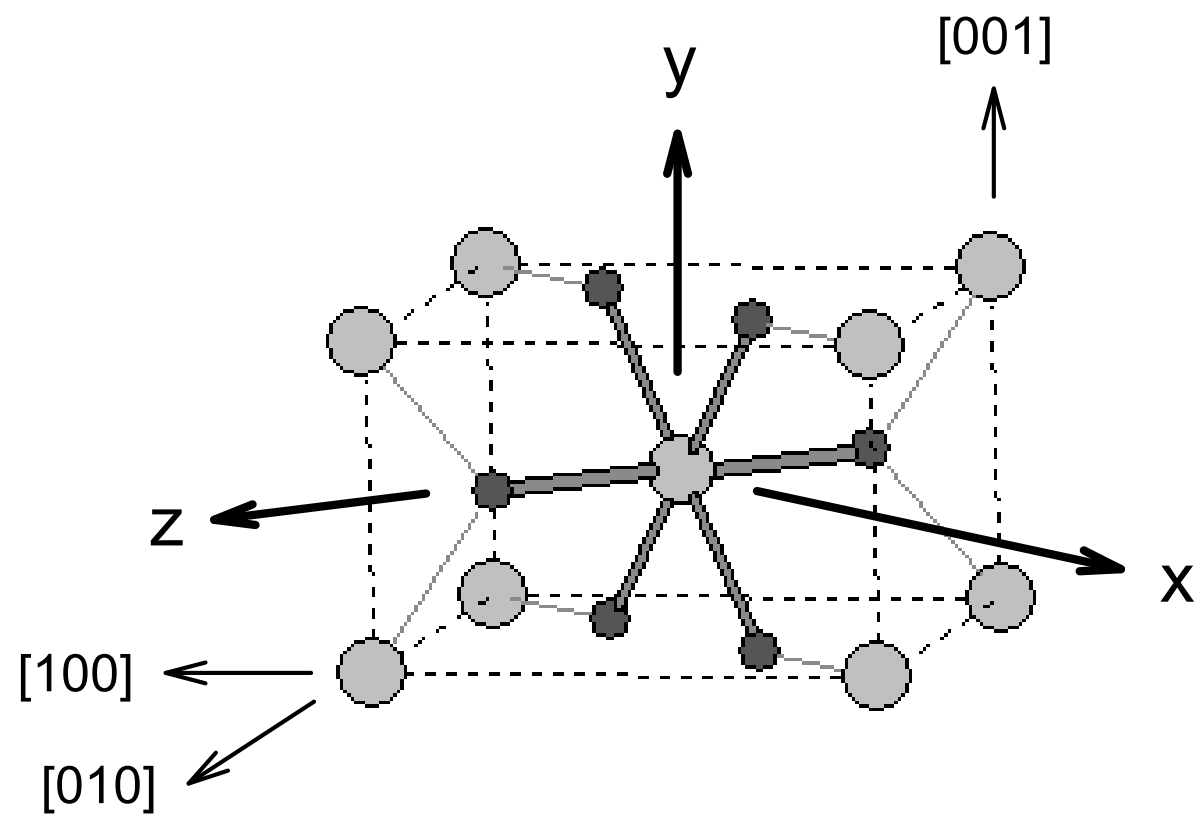

Figure A.1. Illustration of the $\mathrm{TiO}_{6}$ (octahedron) coordinate system in a $\mathrm{TiO}_{2}$ unit cell with rutile structure. 
Figure A.2 shows the electron distribution for the five d orbitals. In the coordinate system showed in Figure A.1, two oxygen ions lie on the $\mathrm{z}$ axis, and for oxygen ions lie in the $\mathrm{Z}=0$ plane. Therefore, an electron in a $d_{z^{2}}$ state on the $\mathrm{Ti}^{3+}$ ion will have a high energy, since the lobes are close to the two oxygen ions on the $\mathrm{z}$ axis. This argument applies to the $d_{x y}$ state as well.

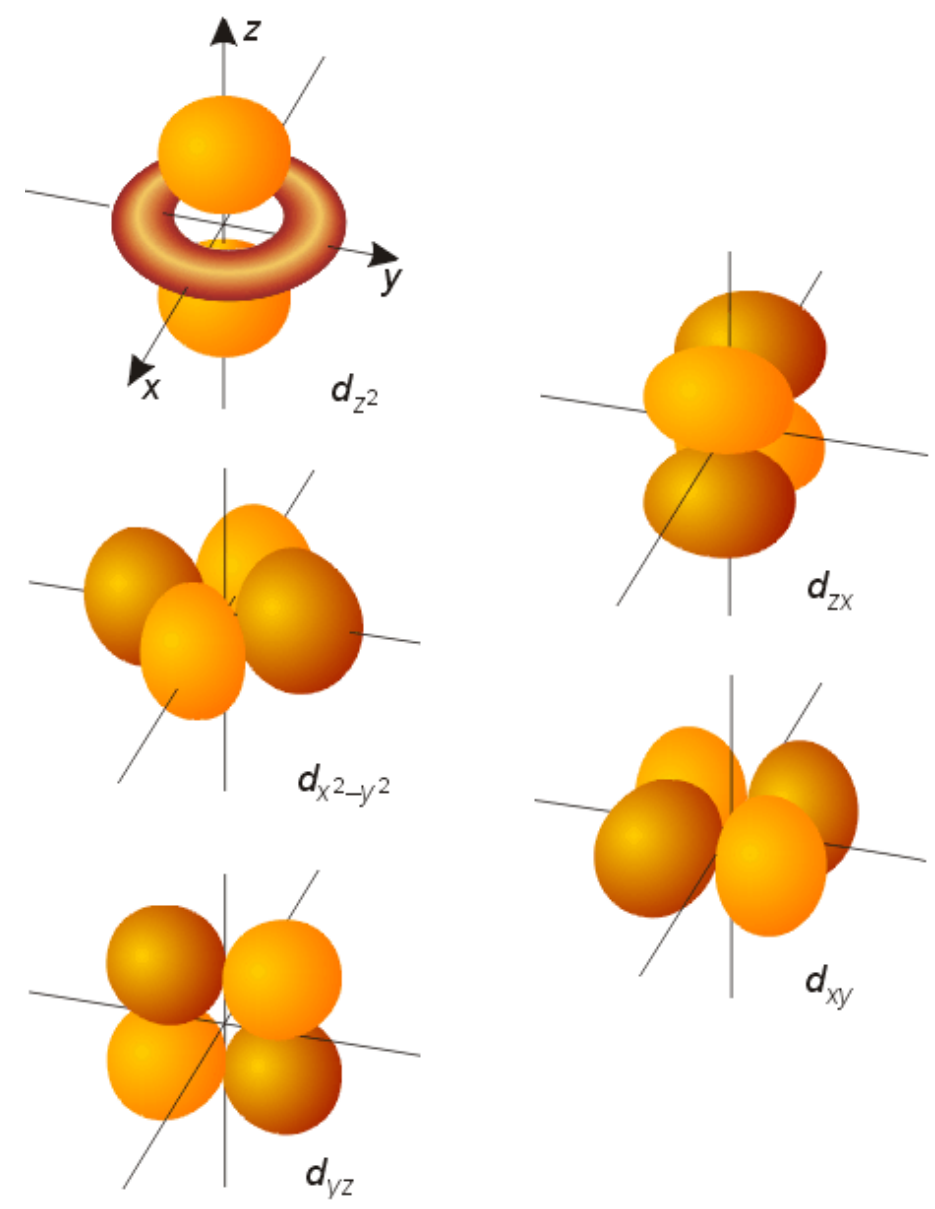

Figure A.2. Representations of the five $\mathrm{d}$ orbitals for a $\mathrm{d}^{1}$ electron in a $\mathrm{Ti}^{3+}$ ion in $\mathrm{TiO}_{2}$. Pictures from Scott Oliver http://www.chemistry.ucsc.edu/ soliver/

Which is the ground state, i.e. which d orbital has the lowest energy in the configuration illustrated in Figure A.1? It is not obvious which of the three states $d_{x z}$, $d_{y z}$ or $d_{x^{2}-y^{2}}$ is the ground state. The $d_{x z}$ and $d_{y z}$ orbitals are very similar to each 
another, so I assume $d_{x^{2}-y^{2}}$ is the ground state first. (I can assume $d_{x z}$ or $d_{y z}$ is the ground state, but the final expressions of the g matrix do not agree with experiment. )

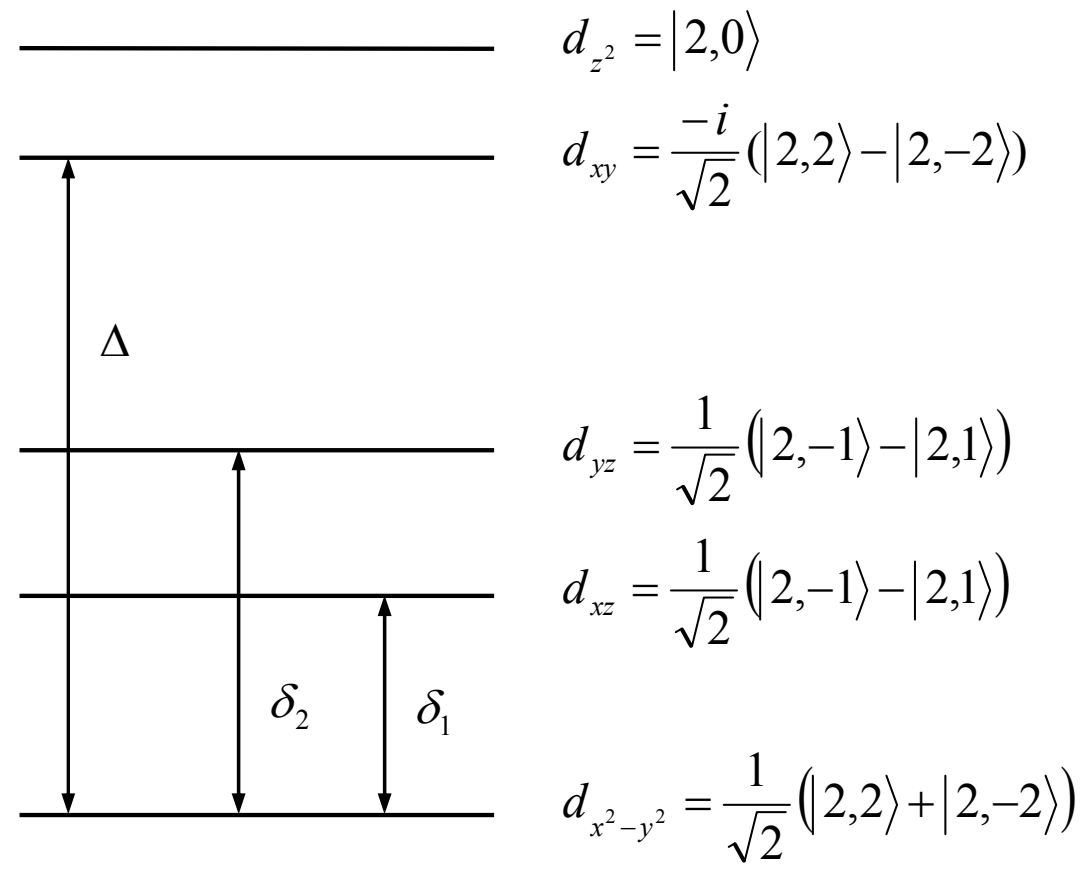

Figure A.3. Energy states splitting of a d-state ion in a crystal field.

Take $d_{x_{2}-y_{2}}=\frac{1}{\sqrt{2}}(|2,2\rangle+|2,-2\rangle)$ as ground, one gets

$$
\Lambda_{x x}=-\sum_{n \neq G} \frac{\left\langle G\left|\hat{L}_{x}\right| n\right\rangle\left\langle n\left|\hat{L}_{x}\right| G\right\rangle}{E_{n}-E_{G}}
$$

Where

$$
\begin{aligned}
\left\langle G\left|\hat{L}_{x}\right| y z\right\rangle=\frac{1}{\sqrt{2}}\left(\left\langle2,2|+\langle 2,-2|) \frac{1}{2}\left|\hat{L}_{+}+\hat{L}_{-}\right| \frac{i}{\sqrt{2}}(|2,-1\rangle+|2,1\rangle)\right.\right. \\
=\frac{i}{4}(\langle 2,2|+\langle 2,-2|)\left(\hat{L}_{+}(|2,-1\rangle+|2,1\rangle)+\hat{L}_{-}(|2,-1\rangle+|2,1\rangle)\right) \\
=\frac{i}{4}(\langle 2,2|+\langle 2,-2|)(\sqrt{6}|2,0\rangle+2|2,2\rangle+2|2,-2\rangle+\sqrt{6}|2,1\rangle) \\
=\frac{i}{4}(2\langle 2,2 \mid 2,2\rangle+2\langle 2,-2 \mid 2,-2\rangle)
\end{aligned}
$$




$$
=i
$$

Similarly, $\left\langle y z\left|\hat{L}_{x}\right| G\right\rangle=-i$

$$
\begin{aligned}
\left\langle G\left|\hat{L}_{x}\right| x z\right\rangle & =\frac{1}{\sqrt{2}}\left(\left\langle2,2|+\langle 2,-2|) \frac{1}{2}\left|\hat{L}_{+}+\hat{L}_{-}\right| \frac{1}{\sqrt{2}}(|2,-1\rangle-|2,1\rangle)\right.\right. \\
& =\frac{1}{4}(\langle 2,2|+\langle 2,-2|)\left(\hat{L}_{+}(|2,-1\rangle-|2,1\rangle)+\hat{L}_{-}(|2,-1\rangle-|2,1\rangle)\right) \\
& =\frac{1}{4}(\langle 2,2|+\langle 2,-2|)(\sqrt{6}|2,0\rangle-2|2,2\rangle+2|2,-2\rangle-\sqrt{6}|2,1\rangle) \\
& =\frac{1}{4}(2\langle 2,2 \mid 2,2\rangle-2\langle 2,-2 \mid 2,-2\rangle) \\
& =0
\end{aligned}
$$

Similarly, $\left\langle x z\left|\hat{L}_{x}\right| G\right\rangle=0$

$$
\begin{aligned}
\left\langle G\left|\hat{L}_{x}\right| x y\right\rangle & =\frac{1}{\sqrt{2}}\left(\left\langle2,2|+\langle 2,-2|) \frac{1}{2}\left|\hat{L}_{+}+\hat{L}_{-}\right| \frac{(-i)}{\sqrt{2}}(|2,2\rangle-|2,-2\rangle)\right.\right. \\
& =\frac{-i}{4}(\langle 2,2|+\langle 2,-2|)\left(\hat{L}_{+}(|2,2\rangle-|2,-2\rangle)+\hat{L}_{-}(|2,2\rangle-|2,-2\rangle)\right) \\
& =\frac{-i}{4}(\langle 2,2|+\langle 2,-2|)(0-2|2,-1\rangle+2|2,2\rangle-0) \\
& =0
\end{aligned}
$$

Similarly, $\left\langle x y\left|\hat{L}_{x}\right| G\right\rangle=0$

$$
\begin{aligned}
\left\langle G\left|\hat{L}_{x}\right| z^{2}\right\rangle & =\frac{1}{\sqrt{2}}\left(\left\langle2,2|+\langle 2,-2|) \frac{1}{2}\left|\hat{L}_{+}+\hat{L}_{-}\right|(|2,0\rangle)\right.\right. \\
& =\frac{\sqrt{6}}{2 \sqrt{2}}(\langle 2,2|+\langle 2,-2|)(|2,1\rangle+|2,-1\rangle) \\
& =0
\end{aligned}
$$

Similarly, $\left\langle z^{2}\left|\hat{L}_{x}\right| G\right\rangle=0$ 
$\Lambda_{x x}=-\sum_{n \neq G} \frac{\left\langle G\left|\hat{L}_{x}\right| n\right\rangle\left\langle n\left|\hat{L}_{x}\right| G\right\rangle}{E_{n}-E_{G}}=-\frac{i^{*}(-i)}{E_{y z}-E_{G}}=-\frac{1}{\delta_{2}}$

Therefore:

$$
g_{x x}=g_{e}-\frac{2 \lambda}{\delta_{2}}
$$

Similarly,

$$
\begin{aligned}
\left\langle G\left|\hat{L}_{y}\right| x z\right\rangle & =i,\left\langle x z\left|\hat{L}_{y}\right| G\right\rangle=-i \\
\left\langle G\left|\hat{L}_{y}\right| x y\right\rangle & =0,\left\langle x y\left|\hat{L}_{y}\right| G\right\rangle=0 \\
\left\langle G\left|\hat{L}_{y}\right| z^{2}\right\rangle & =0,\left\langle z^{2}\left|\hat{L}_{y}\right| G\right\rangle=0 \\
\left\langle G\left|\hat{L}_{z}\right| x y\right\rangle & =\frac{1}{\sqrt{2}}\left(\left\langle2,2|+\langle 2,-2|) \hat{L}_{z} \frac{(-i)}{\sqrt{2}}(|2,2\rangle-|2,-2\rangle)\right.\right. \\
& =\frac{-i}{2}(\langle 2,2|+\langle 2,-2|)(2|2,2\rangle-(-2)|2,-2\rangle) \\
& =\frac{-i}{2}(2\langle 2,2 \mid 2,2\rangle+2\langle 2,-2 \mid 2,-2\rangle) \\
& =-2 i \\
\left\langle G y\left|\hat{L}_{z}\right| G\right\rangle & =2 i \quad\left\langle z^{2}\left|\hat{L}_{z}\right| G\right\rangle=0 \\
\left\langle G\left|\hat{L}_{z}\right| x z\right\rangle & =0 \\
\left\langle G\left|\hat{L}_{z}\right| z^{2}\right\rangle & =0 \\
\left\langle x z\left|\hat{L}_{z}\right| G\right\rangle=0 &
\end{aligned}
$$

$$
g_{z z}=g_{e}-\frac{8 \lambda}{\Delta}
$$

The experimental $g$ values are $g_{x x}=1.9782, g_{y y}=1.9430$, and $g_{z z}=1.9746$, and the spinorbital coupling constant $\lambda$ for $\mathrm{Ti}^{3+}$ ions is $\lambda=154 \mathrm{~cm}^{-1}$. To account for covalency, an orbital reduction factor $k=0.6$ is used and this gives $\lambda^{\prime}=k \lambda=92.4 \mathrm{~cm}^{-1}$. Inserting in these numbers into theses equations derived above, I get: 


$$
\begin{aligned}
& g_{x x}=g_{e}-\frac{2 \lambda^{\prime}}{\delta_{2}} \Rightarrow \delta_{2}=7668 \mathrm{~cm}^{-1} \\
& g_{y y}=g_{e}-\frac{2 \lambda^{\prime}}{\delta_{1}} \Rightarrow \delta_{1}=3116 \mathrm{~cm}^{-1} \\
& g_{z z}=g_{e}-\frac{8 \lambda^{\prime}}{\Delta} \Rightarrow \Delta=26686 \mathrm{~cm}^{-1}
\end{aligned}
$$


Appendix B

Table B.1. EPR angular dependence data of neutral oxygen vacancies.

\begin{tabular}{|c|c|c|c|c|c|c|c|c|c|}
\hline Angle & B1 & B2 & B3 & B4 & B5 & B6 & B7 & B8 & Frequency \\
\hline$[100]$ & 3601.32 & 3647.41 & 3601.32 & 3647.41 & 3313.86 & 3426.11 & 3313.86 & 3426.11 & 9481.108 \\
\hline 0 & 3597.43 & 3641.44 & 3597.43 & 3641.44 & 3332.46 & 3442.28 & 3332.46 & 3442.28 & 9481.050 \\
\hline 10 & 3586.82 & 3625.83 & 3586.82 & 3625.83 & 3382.37 & 3485.75 & 3382.37 & 3485.75 & 9480.735 \\
\hline 20 & 3568.40 & 3599.47 & 3568.40 & 3599.47 & 3464.97 & 3556.55 & 3464.97 & 3556.55 & 9480.199 \\
\hline 30 & 3544.14 & 3569.50 & 3544.14 & 3569.50 & 3566.43 & 3645.11 & 3566.43 & 3645.11 & 9479.717 \\
\hline 40 & 3516.07 & 3530.16 & 3516.07 & 3530.16 & 3684.31 & 3740.64 & 3684.31 & 3740.64 & 9479.120 \\
\hline 50 & 3486.12 & 3493.54 & 3486.12 & 3493.54 & 3801.29 & 3836.41 & 3801.29 & 3836.41 & 9478.627 \\
\hline 60 & 3462.34 & 3465.41 & 3462.34 & 3465.41 & 3891.33 & 3908.93 & 3891.33 & 3908.93 & 9478.374 \\
\hline 70 & 3443.82 & 3444.49 & 3443.82 & 3444.49 & 3958.15 & 3962.25 & 3958.15 & 3962.25 & 9477.820 \\
\hline$[001]$ & 3438.50 & 3438.50 & 3438.50 & 3438.50 & 3977.99 & 3977.99 & 3977.99 & 3977.99 & 9478.044 \\
\hline
\end{tabular}

\begin{tabular}{|c|c|c|c|c|c|c|c|c|c|}
\hline Angle & B1 & B2 & B3 & B4 & B5 & B6 & B7 & B8 & Frequency \\
\hline$[001]$ & 3418.71 & 3418.71 & 3418.71 & 3418.71 & 3958.29 & 3958.29 & 3958.29 & 3958.29 & 9427.452 \\
\hline 10 & 3424.54 & 3424.54 & 3426.16 & 3426.16 & 3937.31 & 3937.31 & 3939.80 & 3939.80 & 9427.570 \\
\hline 20 & 3441.96 & 3441.96 & 3448.70 & 3448.70 & 3871.15 & 3871.15 & 3881.12 & 3881.12 & 9427.012 \\
\hline 30 & 3467.19 & 3467.19 & 3481.24 & 3481.24 & 3776.77 & 3776.77 & 3797.18 & 3797.18 & 9427.774 \\
\hline 40 & 3494.90 & 3494.90 & 3517.19 & 3517.19 & 3669.26 & 3669.26 & 3701.13 & 3701.13 & 9427.852 \\
\hline 50 & 3523.17 & 3523.17 & 3553.96 & 3553.96 & 3557.27 & 3557.27 & 3600.71 & 3600.71 & 9428.530 \\
\hline 60 & 3548.21 & 3548.21 & 3586.48 & 3586.48 & 3456.71 & 3456.71 & 3510.20 & 3510.20 & 9429.740 \\
\hline 70 & 3566.01 & 3566.01 & 3609.89 & 3609.89 & 3382.04 & 3382.04 & 3442.90 & 3442.90 & 9430.286 \\
\hline 80 & 3577.58 & 3577.58 & 3625.01 & 3625.01 & 3332.59 & 3332.59 & 3397.98 & 3397.98 & 9430.500 \\
\hline [110] & 3581.37 & 3581.37 & 3630.03 & 3630.03 & 3316.84 & 3316.84 & 3383.81 & 3383.81 & 9430.738 \\
\hline
\end{tabular}


Table B.1 continued.

\begin{tabular}{|c|c|c|c|c|c|c|c|c|c|}
\hline Angle & B1 & B2 & B3 & B4 & B5 & B6 & B7 & B8 & Frequency \\
\hline$[110]$ & 3594.73 & 3594.73 & 3643.28 & 3643.28 & 3330.21 & 3330.21 & 3397.21 & 3397.21 & 9467.125 \\
\hline 5 & 3591.31 & 3598.91 & 3638.76 & 3647.01 & 3321.51 & 3340.31 & 3387.08 & 3406.66 & 9467.124 \\
\hline 10 & 3588.34 & 3604.23 & 3633.07 & 3650.20 & 3312.80 & 3352.28 & 3374.53 & 3415.60 & 9466.896 \\
\hline 20 & 3585.99 & 3615.20 & 3621.93 & 3652.86 & 3302.79 & 3374.73 & 3352.28 & 3426.76 & 9466.717 \\
\hline 30 & 3587.54 & 3627.10 & 3610.14 & 3651.17 & 3299.93 & 3396.86 & 3331.08 & 3430.47 & 9466.437 \\
\hline 40 & 3592.16 & 3637.22 & 3600.32 & 3645.86 & 3303.64 & 3413.81 & 3314.87 & 3425.79 & 9465.992 \\
\hline 45 & 3595.88 & 3641.87 & 3595.88 & 3641.87 & 3308.48 & 3420.63 & 3308.48 & 3420.63 & 9465.971 \\
\hline 50 & 3600.30 & 3645.95 & 3592.16 & 3637.15 & 3315.10 & 3425.89 & 3303.77 & 3413.81 & 9466.008 \\
\hline 60 & 3610.79 & 3651.14 & 3586.85 & 3625.72 & 3332.14 & 3429.88 & 3299.33 & 3394.94 & 9465.509 \\
\hline 70 & 3622.69 & 3652.14 & 3585.44 & 3613.13 & 3354.26 & 3425.12 & 3302.88 & 3371.48 & 9465.065 \\
\hline 80 & 3633.77 & 3648.62 & 3588.11 & 3601.86 & 3376.93 & 3412.58 & 3313.98 & 3348.09 & 9464.849 \\
\hline 85 & 3638.35 & 3646.60 & 3590.70 & 3598.41 & 3386.25 & 3406.26 & 3320.66 & 3339.80 & 9465.848 \\
\hline$[1-10]$ & 3642.87 & 3642.87 & 3594.24 & 3594.24 & 3396.69 & 3396.69 & 3329.69 & 3329.69 & 9465.823 \\
\hline
\end{tabular}


Table B.2. EPR angular dependence data of singly ionized oxygen vacancies.

\begin{tabular}{|c|c|c|c|c|c|}
\hline Angle & B1 & B2 & B3 & B4 & Frequency \\
\hline$[100]$ & 3529.26 & 3460.35 & 3529.26 & 3460.35 & 9481.108 \\
\hline 10 & 3534.68 & 3467.82 & 3534.68 & 3467.82 & 9481.050 \\
\hline 20 & 3549.88 & 3488.12 & 3549.88 & 3488.12 & 9480.735 \\
\hline 30 & 3574.39 & 3521.07 & 3574.39 & 3521.07 & 9480.199 \\
\hline 40 & 3604.35 & 3561.95 & 3604.35 & 3561.95 & 9479.717 \\
\hline 50 & 3636.11 & 3605.50 & 3636.11 & 3605.50 & 9479.120 \\
\hline 60 & 3667.24 & 3648.92 & 3667.24 & 3648.92 & 9478.627 \\
\hline 70 & 3690.54 & 3681.44 & 3690.54 & 3681.44 & 9478.374 \\
\hline 80 & 3707.27 & 3705.26 & 3707.27 & 3705.26 & 9477.820 \\
\hline$[001]$ & 3712.30 & 3712.30 & 3712.30 & 3712.30 & 9478.044 \\
\hline
\end{tabular}

\begin{tabular}{|c|c|c|c|c|c|}
\hline Angle & B1 & B2 & B3 & B4 & Frequency \\
\hline$[001]$ & 3692.63 & 3692.63 & 3692.63 & 3692.63 & 9427.406 \\
\hline 10 & 3685.86 & 3685.86 & 3685.86 & 3685.86 & 9427.307 \\
\hline 20 & 3664.18 & 3664.18 & 3665.20 & 3665.20 & 9426.940 \\
\hline 30 & 3633.11 & 3633.11 & 3635.31 & 3635.31 & 9427.600 \\
\hline 40 & 3596.76 & 3596.76 & 3600.23 & 3600.23 & 9427.774 \\
\hline 50 & 3558.18 & 3558.18 & 3562.92 & 3562.92 & 9428.484 \\
\hline 60 & 3522.72 & 3522.72 & 3528.63 & 3528.63 & 9429.568 \\
\hline 70 & 3495.77 & 3495.77 & 3502.51 & 3502.51 & 9430.028 \\
\hline 80 & 3477.47 & 3477.47 & 3484.80 & 3484.80 & 9430.060 \\
\hline$[110]$ & 3471.96 & 3471.96 & 3479.40 & 3479.40 & 9430.598 \\
\hline
\end{tabular}

\begin{tabular}{|c|c|c|c|c|c|}
\hline Angle & B1 & B2 & B3 & B4 & Frequency \\
\hline$[110]$ & 3485.49 & 3485.49 & 3492.98 & 3492.98 & 9467.295 \\
\hline 5 & 3491.27 & 3479.47 & 3498.70 & 3486.80 & 9467.114 \\
\hline 10 & 3497.82 & 3473.18 & 3504.89 & 3480.15 & 9466.885 \\
\hline 20 & 3508.70 & 3463.85 & 3514.55 & 3469.46 & 9466.690 \\
\hline 30 & 3517.63 & 3457.36 & 3521.30 & 3460.90 & 9466.467 \\
\hline 40 & 3522.48 & 3454.68 & 3523.82 & 3455.94 & 9466.003 \\
\hline 45 & 3524.04 & 3454.81 & 3524.04 & 3454.81 & 9465.970 \\
\hline 50 & 3523.83 & 3456.03 & 3522.49 & 3454.75 & 9466.001 \\
\hline 60 & 3520.51 & 3461.05 & 3516.61 & 3457.33 & 9465.510 \\
\hline 70 & 3512.85 & 3469.95 & 3506.84 & 3464.20 & 9465.087 \\
\hline 80 & 3502.69 & 3481.24 & 3495.52 & 3474.23 & 9465.275 \\
\hline 85 & 3498.27 & 3486.21 & 3490.83 & 3478.88 & 9465.846 \\
\hline$[1-10]$ & 3492.22 & 3492.22 & 3484.71 & 3484.71 & 9465.816 \\
\hline
\end{tabular}


Table B.3. EPR angular dependence data of $\mathrm{Si}^{4+}-\mathrm{Ti}^{3+}$ center.

\begin{tabular}{|c|c|c|c|}
\hline Angle & B1 & B2 & Frequency \\
\hline$[100]$ & 3488.56 & 3488.56 & 9481.108 \\
\hline 10 & 3488.71 & 3488.71 & 9481.050 \\
\hline 20 & 3489.31 & 3489.31 & 9480.735 \\
\hline 30 & 3490.14 & 3490.14 & 9480.199 \\
\hline 40 & 3491.01 & 3491.01 & 9479.717 \\
\hline 50 & 3491.91 & 3491.91 & 9479.120 \\
\hline 60 & 3492.97 & 3492.97 & 9478.627 \\
\hline 70 & 3493.60 & 3493.60 & 9478.374 \\
\hline 80 & 3494.02 & 3494.02 & 9477.820 \\
\hline$[001]$ & 3494.34 & 3494.34 & 9478.044 \\
\hline
\end{tabular}

\begin{tabular}{|c|c|c|c|}
\hline Angle & B1 & B2 & Frequency \\
\hline$[001]$ & 3475.91 & 3475.91 & 9427.452 \\
\hline 10 & 3474.42 & 3476.91 & 9427.570 \\
\hline 20 & 3469.55 & 3480.25 & 9427.012 \\
\hline 30 & 3463.02 & 3485.76 & 9427.774 \\
\hline 40 & 3454.80 & 3491.97 & 9427.852 \\
\hline 50 & 3446.11 & 3499.11 & 9428.530 \\
\hline 60 & 3438.01 & 3506.24 & 9429.740 \\
\hline 70 & 3431.67 & 3511.57 & 9430.286 \\
\hline 80 & 3427.05 & 3514.97 & 9430.500 \\
\hline$[110]$ & 3425.77 & 3516.28 & 9430.738 \\
\hline
\end{tabular}

\begin{tabular}{|c|c|c|c|}
\hline Angle & B1 & B2 & Frequency \\
\hline$[110]$ & 3438.96 & 3529.68 & 9467.125 \\
\hline 5 & 3439.54 & 3529.01 & 9467.124 \\
\hline 10 & 3441.79 & 3526.58 & 9466.896 \\
\hline 20 & 3449.48 & 3518.18 & 9466.717 \\
\hline 30 & 3461.77 & 3505.32 & 9466.437 \\
\hline 40 & 3475.41 & 3491.03 & 9465.992 \\
\hline 45 & 3483.19 & 3483.19 & 9465.971 \\
\hline 50 & 3491.14 & 3475.30 & 9466.008 \\
\hline 60 & 3506.12 & 3460.28 & 9465.509 \\
\hline 70 & 3518.82 & 3447.71 & 9465.065 \\
\hline 80 & 3526.23 & 3440.21 & 9464.849 \\
\hline 85 & 3528.49 & 3439.10 & 9465.848 \\
\hline$[1-10]$ & 3529.16 & 3438.38 & 9465.823 \\
\hline
\end{tabular}


Table B.4. EPR angular dependence data of self-trapped $\mathrm{Ti}^{3+}$ center.

\begin{tabular}{|c|c|c|c|}
\hline Angle & B1 & B2 & Frequency \\
\hline$[100]$ & 3417.29 & 3417.29 & 9466.857 \\
\hline 10 & 3418.32 & 3418.32 & 9466.380 \\
\hline 20 & 3422.05 & 3422.05 & 9466.740 \\
\hline 30 & 3427.56 & 3427.56 & 9467.634 \\
\hline 40 & 3434.60 & 3434.60 & 9468.530 \\
\hline 50 & 3441.73 & 3441.73 & 9468.698 \\
\hline 60 & 3448.66 & 3448.66 & 9469.282 \\
\hline 70 & 3454.16 & 3454.16 & 9469.599 \\
\hline 80 & 3457.90 & 3457.90 & 9469.823 \\
\hline$[001]$ & 3459.07 & 3459.07 & 9469.900 \\
\hline
\end{tabular}

\begin{tabular}{|c|c|c|c|}
\hline Angle & B1 & B2 & Frequency \\
\hline$[001]$ & 3442.55 & 3442.55 & 9424.738 \\
\hline 10 & 3441.39 & 3441.39 & 9424.481 \\
\hline 20 & 3438.19 & 3438.44 & 9425.052 \\
\hline 30 & 3432.69 & 3433.36 & 9425.498 \\
\hline 40 & 3425.52 & 3426.91 & 9425.120 \\
\hline 50 & 3418.42 & 3420.46 & 9425.648 \\
\hline 60 & 3411.48 & 3414.13 & 9426.200 \\
\hline 70 & 3405.99 & 3409.11 & 9426.543 \\
\hline 80 & 3402.21 & 3405.67 & 9426.864 \\
\hline$[110]$ & 3401.04 & 3404.63 & 9427.059 \\
\hline
\end{tabular}

\begin{tabular}{|c|c|c|c|}
\hline Angle & B1 & B2 & Frequency \\
\hline$[110]$ & 3419.14 & 3415.54 & 9467.122 \\
\hline 5 & 3419.09 & 3415.53 & 9467.035 \\
\hline 10 & 3418.96 & 3415.61 & 9466.972 \\
\hline 20 & 3418.60 & 3415.90 & 9466.800 \\
\hline 30 & 3418.03 & 3416.35 & 9466.531 \\
\hline 40 & 3417.53 & 3416.96 & 9466.506 \\
\hline 45 & 3417.17 & 3417.17 & 9466.370 \\
\hline 50 & 3416.76 & 3417.48 & 9466.201 \\
\hline 60 & 3416.00 & 3417.90 & 9465.863 \\
\hline 70 & 3415.25 & 3418.11 & 9465.353 \\
\hline 80 & 3414.86 & 3418.30 & 9465.175 \\
\hline 85 & 3414.81 & 3418.41 & 9465.267 \\
\hline$[1-10]$ & 3414.75 & 3418.37 & 9465.177 \\
\hline
\end{tabular}


Table B.5. EPR angular dependence data of self-trapped hole center.

\begin{tabular}{|c|c|c|c|}
\hline Angle & B1 & B2 & Frequency \\
\hline$[001]$ & 3345.30 & 3345.30 & 9424.737 \\
\hline 10 & 3344.56 & 3345.61 & 9424.493 \\
\hline 20 & 3342.73 & 3347.06 & 9425.047 \\
\hline 30 & 3339.69 & 3349.18 & 9425.495 \\
\hline 40 & 3335.56 & 3351.54 & 9425.130 \\
\hline 50 & 3331.51 & 3354.23 & 9425.653 \\
\hline 60 & 3327.57 & 3356.91 & 9426.194 \\
\hline 70 & 3324.41 & 3358.97 & 9426.550 \\
\hline 80 & 3322.26 & 3360.45 & 9426.857 \\
\hline$[110]$ & 3321.57 & 3360.90 & 9427.063 \\
\hline
\end{tabular}

\begin{tabular}{|c|c|c|c|}
\hline Angle & B1 & B2 & Frequency \\
\hline$[110]$ & 3335.75 & 3375.22 & 9467.112 \\
\hline 5 & 3336.15 & 3374.77 & 9467.037 \\
\hline 10 & 3337.14 & 3373.68 & 9466.974 \\
\hline 15 & 3338.60 & 3372.19 & 9466.897 \\
\hline 20 & 3340.57 & 3370.11 & 9466.797 \\
\hline 25 & 3343.03 & 3367.45 & 9466.680 \\
\hline 30 & 3346.04 & 3364.47 & 9466.532 \\
\hline 35 & 3349.26 & 3361.16 & 9466.400 \\
\hline 40 & 3351.83 & 3358.69 & 9466.497 \\
\hline 45 & 3355.15 & 3355.15 & 9466.375 \\
\hline 50 & 3358.62 & 3351.37 & 9466.202 \\
\hline 55 & 3362.39 & 3347.71 & 9466.058 \\
\hline 60 & 3365.41 & 3344.64 & 9465.880 \\
\hline 65 & 3367.98 & 3341.89 & 9465.709 \\
\hline 70 & 3370.44 & 3339.21 & 9465.355 \\
\hline 75 & 3372.15 & 3337.47 & 9465.273 \\
\hline 80 & 3373.52 & 3336.07 & 9465.173 \\
\hline 85 & 3374.35 & 3335.30 & 9465.262 \\
\hline$[1-10]$ & 3374.50 & 3335.03 & 9465.171 \\
\hline
\end{tabular}


Table B.6. EPR angular dependence data of fluorine $(\mathrm{F})$ center in $\mathrm{TiO}_{2}$.

\begin{tabular}{|c|c|c|c|c|c|}
\hline Angle & B1 & B2 & B3 & B4 & Frequency \\
\hline & & & & & \\
\hline$[100]$ & 3424.55 & 3424.55 & 3424.55 & 3424.55 & 9472.859 \\
\hline 10 & 3426.38 & 3426.38 & 3426.38 & 3426.38 & 9473.151 \\
\hline 20 & 3431.23 & 3431.23 & 3431.53 & 3431.53 & 9473.448 \\
\hline 30 & 3438.77 & 3438.77 & 3439.18 & 3439.18 & 9473.512 \\
\hline 40 & 3448.30 & 3448.30 & 3448.95 & 3448.95 & 9474.117 \\
\hline 50 & 3458.68 & 3458.68 & 3459.68 & 3459.68 & 9474.738 \\
\hline 60 & 3468.64 & 3468.64 & 3470.01 & 3470.01 & 9475.232 \\
\hline 70 & 3476.59 & 3476.59 & 3478.21 & 3478.21 & 9475.584 \\
\hline 80 & 3481.77 & 3481.77 & 3483.51 & 3483.51 & 9475.725 \\
\hline$[001]$ & 3483.53 & 3483.53 & 3485.31 & 3485.31 & 9475.939 \\
\hline
\end{tabular}

\begin{tabular}{|c|c|c|c|c|c|}
\hline Angle & B1 & B2 & B3 & B4 & Frequency \\
\hline$[001]$ & 3484.18 & 3484.18 & 3485.96 & 3485.96 & 9477.783 \\
\hline 10 & 3482.39 & 3482.39 & 3484.15 & 3484.15 & 9477.735 \\
\hline 20 & 3476.83 & 3477.57 & 3478.46 & 3479.03 & 9477.575 \\
\hline 30 & 3468.66 & 3470.07 & 3470.07 & 3471.39 & 9477.227 \\
\hline 40 & 3457.68 & 3460.36 & 3458.83 & 3461.35 & 9476.949 \\
\hline 50 & 3446.79 & 3450.64 & 3447.72 & 3451.27 & 9476.560 \\
\hline 60 & 3437.26 & 3442.13 & 3438.00 & 3442.52 & 9476.194 \\
\hline 70 & 3429.21 & 3434.86 & 3429.67 & 3434.86 & 9476.860 \\
\hline 80 & 3424.35 & 3430.37 & 3424.45 & 3430.37 & 9475.666 \\
\hline$[110]$ & 3422.37 & 3428.56 & 3422.37 & 3428.56 & 9475.560 \\
\hline
\end{tabular}

\begin{tabular}{|c|c|c|c|c|c|}
\hline Angle & B1 & B2 & B3 & B4 & Frequency \\
\hline$[110]$ & 3417.07 & 3423.24 & 3417.07 & 3423.24 & 9461.018 \\
\hline 5 & 3417.16 & 3423.19 & 3417.16 & 3423.19 & 9461.013 \\
\hline 10 & 3417.26 & 3423.02 & 3417.26 & 3423.02 & 9461.000 \\
\hline 20 & 3417.79 & 3422.53 & 3417.79 & 3422.53 & 9460.939 \\
\hline 30 & 3418.70 & 3421.72 & 3418.70 & 3421.72 & 9460.850 \\
\hline 40 & 3419.66 & 3420.68 & 3419.66 & 3420.68 & 9460.640 \\
\hline 45 & 3420.21 & 3420.21 & 3420.21 & 3420.21 & 9460.627 \\
\hline 50 & 3420.70 & 3419.70 & 3420.70 & 3419.70 & 9460.642 \\
\hline 60 & 3421.58 & 3418.60 & 3421.58 & 3418.60 & 9460.390 \\
\hline 70 & 3422.28 & 3417.65 & 3422.28 & 3417.65 & 9460.177 \\
\hline 80 & 3422.70 & 3417.04 & 3422.70 & 3417.04 & 9460.016 \\
\hline 85 & 3422.90 & 3416.90 & 3422.90 & 3416.90 & 9460.050 \\
\hline$[1-10]$ & 3422.95 & 3416.80 & 3422.95 & 3416.80 & 9460.032 \\
\hline
\end{tabular}


Table B.7. ENDOR angular dependence data of fluorine (F) center in $\mathrm{TiO}_{2}$.

\begin{tabular}{|c|c|c|c|c|c|c|c|c|c|c|}
\hline Angle & Site1 (H) & & & & Site 2 $(\mathbf{H})$ & & & Site 3 (H) & & \\
\hline$[100]$ & 3428.27 & 13.585 & 13.901 & & 3428.27 & 13.585 & 13.901 & & & \\
\hline 10 & 3429.85 & 13.466 & 14.075 & & 3429.85 & 13.682 & 13.810 & & & \\
\hline 20 & 3434.70 & 13.204 & 14.402 & & 3434.70 & 13.640 & 13.907 & & & \\
\hline 30 & 3442.13 & 12.876 & 14.823 & & 3442.13 & 13.481 & 14.158 & & & \\
\hline 40 & 3451.67 & 12.489 & 15.302 & & 3451.67 & 13.218 & 14.539 & & & \\
\hline 50 & 3461.99 & 12.135 & 15.727 & & 3461.99 & 12.898 & 14.972 & & & \\
\hline 60 & 3471.12 & 11.799 & 16.110 & & 3471.12 & 12.499 & 15.440 & & & \\
\hline 70 & 3479.95 & 11.546 & 16.384 & & 3479.95 & 12.087 & 15.906 & & & \\
\hline 80 & 3485.03 & 11.466 & 16.494 & & 3485.03 & 11.784 & 16.220 & & & \\
\hline$[001]$ & 3487.24 & 11.529 & 16.444 & & 3487.24 & 11.529 & 16.444 & & & \\
\hline
\end{tabular}

\begin{tabular}{|c|c|c|c|c|c|c|c|c|c|}
\hline Angle & Site1 (H) & & & Site $2(\mathrm{H})$ & & & Site $3(\mathbf{H})$ & & \\
\hline [001] & 3491.59 & 11.547 & 16.465 & 3491.59 & 11.547 & 16.465 & & & \\
\hline 10 & 3490.10 & 11.427 & 16.579 & 3489.67 & 11.631 & 16.384 & 3490.10 & 11.825 & 16.212 \\
\hline 20 & 3486.62 & 11.425 & 16.539 & 3485.87 & 11.864 & 16.153 & 3485.17 & 12.170 & 15.876 \\
\hline 30 & & & & 3476.46 & 12.194 & 15.772 & & & \\
\hline 40 & & & & 3467.15 & 12.582 & 15.341 & & & \\
\hline 50 & 3454.85 & 12.113 & 15.669 & 3457.45 & 12.978 & 14.862 & 3454.85 & 13.171 & 14.593 \\
\hline 60 & & & & 3448.69 & 13.338 & 14.405 & & & \\
\hline 70 & & & & 3440.92 & 13.610 & 14.010 & & & \\
\hline 80 & 3431.18 & 13.148 & 14.411 & 3437.27 & 13.817 & 13.762 & 3431.18 & 13.503 & 14.003 \\
\hline [110] & 3427.83 & 13.376 & 14.114 & & & & & & \\
\hline
\end{tabular}


Table B.7. Continued.

\begin{tabular}{|c|c|c|c|c|c|c|c|c|c|c|}
\hline Angle & Site1 (H) & & & & Site 2 $(\mathbf{H})$ & & & Site 3 (H) & & \\
\hline$[110]$ & 3433.87 & 13.381 & 14.135 & & 3440.06 & 13.888 & 13.682 & & & \\
\hline 10 & 3434.05 & 13.403 & 14.116 & & 3439.73 & 13.881 & 13.696 & & & \\
\hline 20 & 3434.65 & 13.452 & 14.074 & & & & & & & \\
\hline 30 & 3435.67 & 13.528 & 14.010 & & & & & & & \\
\hline 40 & 3436.84 & 13.610 & 13.940 & & & & & & & \\
\hline 45 & 3437.17 & 13.654 & 13.902 & & & & & & & \\
\hline 50 & & & & & 3436.36 & 13.592 & 13.961 & & & \\
\hline 60 & & & & & 3435.54 & 13.512 & 14.028 & & & \\
\hline 70 & & & & & 3434.66 & 13.441 & 14.093 & & & \\
\hline 80 & & & & & 3434.03 & 13.412 & 14.129 & & & \\
\hline$[1-10]$ & 3440.03 & 13.677 & 13.892 & & 3433.92 & 13.394 & 14.132 & & & \\
\hline
\end{tabular}


Table B.8. EPR angular dependence data of the interstitial Na center.

\begin{tabular}{|c|c|c|c|c|c|c|c|c|c|}
\hline Angle & B1 & B2 & B3 & B4 & B5 & B6 & B7 & B8 & Frequency \\
\hline$[001]$ & 3466.50 & & 3466.50 & & 3898.45 & & 3898.45 & & 9469.968 \\
\hline 0 & 3468.58 & & 3472.82 & & 3878.08 & & 3883.97 & & 9469.903 \\
\hline 10 & 3482.41 & & 3486.74 & & 3817.59 & & 3839.79 & & 9469.792 \\
\hline 20 & 3498.52 & & 3508.40 & & 3732.38 & & 3777.15 & & 9469.598 \\
\hline 30 & 3515.93 & & 3533.55 & & 3634.21 & & 3703.40 & & 9469.407 \\
\hline 40 & 3532.40 & & 3559.11 & & 3532.40 & & 3627.03 & & 9468.900 \\
\hline 50 & 3545.90 & & 3581.90 & & 3445.44 & & 3556.85 & & 9468.520 \\
\hline 60 & 3555.43 & & 3599.64 & & 3375.75 & & 3501.22 & & 9467.445 \\
\hline 70 & 3561.45 & & 3611.14 & & 3332.14 & & 3466.25 & & 9467.720 \\
\hline$[100]$ & 3563.18 & & 3614.78 & & 3316.21 & & 3453.27 & & 9467.212 \\
\hline
\end{tabular}

\begin{tabular}{|c|c|c|c|c|c|c|c|c|c|}
\hline Angle & B1 & B2 & B3 & B4 & B5 & B6 & B7 & B8 & Frequency \\
\hline$[100]$ & 3556.45 & 3556.45 & 3607.81 & 3607.81 & 3311.56 & 3311.56 & 3447.97 & 3447.97 & 9477.215 \\
\hline 5 & 3553.67 & 3560.15 & 3604.05 & 3610.89 & 3310.95 & 3313.52 & 3445.55 & 3448.36 & 9477.214 \\
\hline 10 & 3551.59 & 3564.80 & 3599.52 & 3613.33 & 3313.27 & 3318.28 & 3441.14 & 3446.27 & 9477.500 \\
\hline 20 & 3550.66 & 3574.75 & 3589.66 & 3615.09 & 3322.26 & 3331.41 & 3427.26 & 3437.04 & 9478.001 \\
\hline 30 & 3552.88 & 3585.51 & 3579.16 & 3612.91 & 3337.18 & 3349.64 & 3408.21 & 3421.04 & 9478.587 \\
\hline 40 & 3559.42 & 3597.69 & 3567.73 & 3606.49 & 3359.85 & 3374.40 & 3382.58 & 3397.13 & 9479.173 \\
\hline 45 & 3563.34 & 3602.33 & 3563.34 & 3602.33 & 3371.34 & 3386.14 & 3371.34 & 3386.14 & 9479.484 \\
\hline 50 & 3566.98 & 3605.86 & 3560.02 & 3598.40 & 3380.62 & 3395.28 & 3361.67 & 3376.09 & 9479.840 \\
\hline 60 & 3576.73 & 3612.07 & 3554.11 & 3588.46 & 3402.84 & 3416.05 & 3341.59 & 3354.67 & 9480.328 \\
\hline 70 & 3589.29 & 3615.57 & 3551.26 & 3576.34 & 3426.51 & 3436.29 & 3323.70 & 3333.35 & 9480.694 \\
\hline 80 & 3599.69 & 3614.47 & 3552.35 & 3566.17 & 3441.06 & 3446.79 & 3314.31 & 3319.56 & 9480.897 \\
\hline 85 & 3604.71 & 3611.92 & 3554.45 & 3561.18 & 3446.57 & 3449.51 & 3312.47 & 3314.92 & 9480.951 \\
\hline$[010]$ & 3608.88 & 3608.88 & 3557.52 & 3557.52 & 3448.92 & 3448.92 & 3312.35 & 3312.35 & 9480.942 \\
\hline
\end{tabular}

